UNIVERSITE DU QUEBEC A CHICOUTIMI

\title{
PETROGRAPHIE ET GEOCHIMIE DU COMPLEXE IGNE \\ ALCALIN DE CREVIER ET DE SON ENCAISSANT \\ METASOMATISE
}

PAR

ALAIN BERGERON

THESE PRESENTEE A CHICOUTIMI EN VUE DE

L'OBTENTION D'UNE MAITRISE EN SCIENCE

APPLIQUE (GEOLOGIE)

AUTOMNE I980 


\section{Bibliothèque}

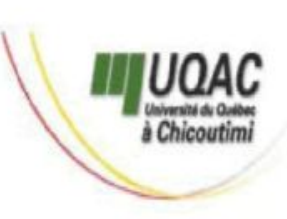

\section{Mise en garde/Advice}

Afin de rendre accessible au plus Motivated by a desire to make the grand nombre le résultat des results of its graduate students' travaux de recherche menés par ses research accessible to all, and in étudiants gradués et dans l'esprit des accordance with the rules règles qui régissent le dépôt et la governing the acceptation and diffusion des mémoires et thèses diffusion of dissertations and produits dans cette Institution, theses in this Institution, the I'Université du Québec à Université du Québec à Chicoutimi (UQAC) est fière de Chicoutimi (UQAC) is proud to rendre accessible une version make a complete version of this complète et gratuite de cette œuvre. work available at no cost to the reader.

L'auteur conserve néanmoins la The author retains ownership of the propriété du droit d'auteur qui copyright of this dissertation or protège ce mémoire ou cette thèse. thesis. Neither the dissertation or Ni le mémoire ou la thèse ni des thesis, nor substantial extracts from extraits substantiels de ceux-ci ne it, may be printed or otherwise peuvent être imprimés ou autrement reproduced without the author's reproduits sans son autorisation. permission. 


\section{TABLE DES MATIERES}

INTRODUCTION

p. 7

I)- Géologie générale

p. $?$

2)- Travaux antērieurs

p. 3

3)- Buts et méthodes de travail

p. 4

I- GEOLOGIE DU COMPLEXE

p. 5

I. I Pétrographie

p. 5

I. I-I Nomenclature

p. 5

I.I-2 Les monzosyénites

p. 8

I. I-3 Les diorites à biotite

I. I-4 Les monzosyénites à néphêtine

p. 13

a) - Les monzosyênites à néphêtine I

p. 13

b) - Les monzosyênites à néphéline pegmatitique II

p. 15

c)- Les monzosyēnites à nêphēiline et biotite III

p. 16

d) - Les monzosyēnites à néphêtine IV

p. 17

I. I-5 Les carbonatites

p. 79

I.2 Grandes unités et structure du complexe

p. 25

I.2-I L'unité I

p. 25

I.2-2 L'unité 2

p. 28

I.2-3 L'unitê 3

p. 29

I.2-4 L'unité 4

p. 31

I. 3 Succession chronologique des évênements intrusifs

p. 34

II- Géologie de la roche encaissante

p. 45

2. I Structure et relations du contact pluton-encaissant

p. 45

2.2 Pétrographie des principaux façiès

p. 46 
2.2-I Gneiss oeillès

2.2-2 Gneiss quartzo-feldspathique à biotite et hornblende

2.2-3 Quartzites

III- GEOCHIMIE DU COMPLEXE

3. I Présentation des résultats

a)- Diagramme A-F-M

b) - Diaggramme $\mathrm{C}-\mathrm{N}-\mathrm{K}$

p. 63

c)- Diagramme QZ-NE-KS- et QZ-AN-KS

p. 65

IV- GEOCHIMIE DES FENITES

p. 70

4. I Evaluation qualitative du métasomatisme

p. 70
a) - Diagramme A-F-M
p. 71
b) - Diagramme $\mathrm{C}-\mathrm{N}-\mathrm{K}$
p. 77
c) - Diagramme QZ-NE-KS
p. 74

4.2 Evaluation quantitative du métasomatisme

4.2-I Méthode de calcul

4.2-2 Prēsentation des données

p. 78

4.2-3 Présentation des résultats

a) - Evaluation de $F_{v}$

b) - Evaluation des gains et des pertes

c)- Diagramme des gains et pertes en fonction de la position des êchantillons

4.3 Evaluation du métasomatisme 
-IV-

ANNEXE I

p. 107

ANNEXE II

p. 108

ANNEXE III

p. 109

ANNEXE IV

p. 109

ANNEXE $V$

p. 111

ANNEXE VI

p. 112

ANNEXE VII

p. 117

ANNEXE VIII

p. 123

REFERENCES

p. 126

CONTENU DES ANNEXES

p.V

LISTE DES FIGURES

p.VI

LISTE DES TABLEAUX

p.VIII

LISTE DES PLANCHES

p.IX

RESUME

p.XI 
ANNEXE I : Méthode d'échantillonage

ANNEXE II : Méthode d'anatyse

ANNEXE III : Schêma de broyage

ANNEXE IV : Méthode de calcul de la densité des pegmatites

ANNEXE $V$ : Détermination de la calcicité plagioclases

ANNEXE VI : Exemple de calcul de l'évaluation quantitative du métasomatisme

ANNEXE VII : Résultats d'analyse et du calcul de la norme des roches du complexe de Crevier

ANNEXE VIII: Résultats d'analyse et du calcul de la norme des pegmatites 
FIGURE I : Localisation du complexe de Crevier et illustration du système de rift du Saint-Laurent

FIGURE 2 : Le complexe igné alcalin de Crevier et ses principales unitès

FIGURE 3 : Affleurement type de T'unité I

FIGURE 4 : Affieurement type de 7 'unité 2

FIGURE 5 : Réseau hydrographique de la région de la rivière Mistassini et principales structures

FIGUREः6 : Diagramme A-F-M des roches du complexe de Crevier

FIGURE 7 : Diagramme $\mathrm{C}-\mathrm{N}-\mathrm{K}$ des roches du complexe de Crevier

FIGURE 8 : Diagramme QZ-NE-KS des roches du complexe de Crevier

FIGURE 9 : Diagramme QZ-AN-KS des roches du complexe de Crevier

FIGURE I0: Diagramme A-F-M des pegmatites de 1 'encaissant du complexe de Crevier

FIGURE II: Diagramme $\mathrm{C}-\mathrm{N}-\mathrm{K}$ des pegmatites de $\mathrm{l}$ 'encaissant du complexe de Crevier

FIGURE I2: Diagramme QZ-NE-KS des pegmatites de T'encaissant du complexe de Crevier

FIGURE I3: Diagramme $C-\forall$, échantilion I

FIGURE I4: Diagramme $C-V$, échantilions 2 et 3

FIGURE 15: Diagramme $C-V$, échantilions 4 et 5

FIGURE I6: Diagramme $C-V$, échantilions 6 et 7

FIGURE I7: Diagramme $C-V$, échantillons 8 et 9

FIGURE I8: Diagramme $C-V$, échantilions IO et II

FIGURE 19: Diagrammes des gains et pertes en fonction de la po- 
sition des échantilions sur la coupe d'échantillonage

FIGURE 20: Schéma illustrant les effets du métasomatisme sur les roches de T'encaissant du complexe de Crevier. 


\section{LISTE DES TABLEAUX}

TABLEAU I : Pourcentage des différents minéraux détérminés par analyse modale au compteur de points

TABLEAU 2 : Tableau résumant la succession chronologique des événements intrusifs

TABLEAU 3 : Analyses modifiées pour l'étude des pegmatites

TABLEAU 4 : Taux de variation maximum des éléments dans les roches métasomatisées

TABLEAU 5 : Valeurs de $F_{v}$ pour les différents axides à $x_{n}=0$

TABLEAU 6 : Valeurs des gains et des pertes calculées à partir de T'équation de Gresens, pour les élêments majeurs et mineurs des pegmatites métasomatisées de l'encaissant du complexe de Crevier 


\section{LISTE DES PLANCHES}

PLANCHE I : Microphotographie d'une monzosyénite

PLANCHE 2 : Microphotographie d'une diorite à biotite

PLANCHE 3 : Microphotographie d'une monzosyênite à nêphēline I

PLANCHE 4 : Microphotographie de 1'altération en cancrinite d'une monzosyénite à néphēline

PLANCHE 5 : Microphotographie d'une monzosyénite à néphēline pegmatitique

PLANCHE 6 : Photographie illustrant la texture mégascopique d'une monzosyênite à néphéline pegmatitique

PLANCHE 7 : Microphotographie d'une monzosyénite à néphéline IV

PLANCHE 8 : Microphotographie d'une carbonatite

PLANCHE 9 : Photographie de la disposition des phénocristaux de néphêtine et d'orthose dans certaines bandes de monzosyénite à néphēline I.

PLANCHE I6: Photographie d'un gneiss oejllê digéré par la monzosyēnite à néphéline I

PLANCHE II: Photographie des veinules d'aégyrine recoupant une enclave de gneiss oeillé

PLANCHE 12: Photographie d'une enclave de monzosyénite à néphéline I dans la diorite à biotite

PLANCHE 13: Photographie d'une enclave de diorite à biotite dans la monzosyênite à néphẻ]ine I

PLANCHE I4: Photographie des relations stratigraphiques dans l'unité 4

PLANCHE 15: Photographie des relations stratigraphiques dans l'unité 2

PLANCHE 16: Photographie des relations stratigraphiques dans 1'unité 2 
PLANCHE I7: Photographie des relations stratigraphiques dans l'unité I

PLANCHE I8: Photographie des relations stratigraphiques de l'unité I

PLANCHE 19: Photographie illustrant la digestion de roches par la carbonatite

PLANCHE 20: Photographie d'une zone de broyage

PLANCHE 2I: Photographie de la texture générale d'une pegmatite nonmétasomatisée

PLANCHE 22: Microphotographie d'une pegmatite non-métasomatisêe

PLANCHE 23: Photographie d'une pegmatite faiblement métasomatisée

PLANCHE 24: Microphotographie d'une pegmatite faiblement métasomatisée

PLANCHE 25: Photographie d'une pegmatite métasomatisée

PLANCHE 26: Microphotographie d'une pegmatite métasomatisée. 
RESUME

Le complexe igné alcalin de Crevier constitue une intrusion post-orogénique, qui s'est mise en place à travers des métasêdiments migmatisés à divers degrês de la ceinture centrale de gneiss de la province géologique du Grenville. Nous avons identifié quatre types de roche, soit: les monzosyénites à néphéline, les monzosyénites, les carbonatites et les diorites à biotite. Les monzosyénites à néphéline sont sub-divisées en quatre sous facies et ce d'après leur morphologie et leur mode de mise en place lors de l'intrusion du massif. Des indices minéralisés en tantale-niobium et uranium-niobium,sont associés respectivement aux monzosyénites à néphéline pegmatitique et à la carbonatite.

Le complexe igné alcalin de Crevier ne comporte que des structures linéaires, contrairement aux structures en anneaux concentriques de la majorité des intrusions de ce type. L'orientation des structures linéaires du complexe transgresse celles mesurées dans l'encaissant.

L'étude pétrogéochimique des roches du complexe nous a permis d'idenfitier une légère différenciation des principaux types de roches; nous avons aussi évalué à partir des échantilions les plus différenciés, la pression à laquelle ces roches se sont mises en place, soit une pression de vapeur d'eau de l'ordre de $8 \mathrm{~kb}$ ou $30 \mathrm{~km}$ de profondeur. Suite à cette étude pétrochimique, nous proposons deux hypothèses probables quant à l'origine des roches du complexe: une cristallisation fractionnée provenant du manteau ou fusion partielle d'un matériel approprié au niveau de la croute inférieure. Nous ne pouvons pas déterminer la source exacte car au niveau actuel d'érosion du complexe, nous n'observons pas 
de membres basiques issus d'une cristallisation fractionnée au niveau du manteau. Des études isotopiques du räbidium-strontium,seraient nécessaires pour lever le doute.

L'intrusion du massif a produit la fénitisation (ou métasomatisme) de la roche encaissante. Le métasomatisme s'observe surtout par l'aégyrinisation des gneiss et des pegmatites, formant une auréole de 2 à $4 \mathrm{~km}$ de largeur ceinturant le massif igné. Nous avons identifié quatre épisodes métasomatiques. Les trois premiers sont reliés à l'intrusion des monzosyénites à néphéline. Ils sont responsables de l'aégyrinisation des roches de l'encaissant et constituent les premiers événements dans la succession chronologique, reliés à l'intrusion du complexe alcalin. Le quatrième épisode métasomatique est relié à l'intrusion de la carbonatite, dernier évenement intrusif observé sur le tẻrrain.

Nous avons évalué les effets de la fénitisation sur l'assemblage minéralogique des pegmatites post-orogéniques qui traversent la zone métasomatisēe, ainsi que l'évaluation qualitative et quantitative des transfers de masse qui ont été produits et enfin la variation de volume résultant de la fénitisation. Au niveau de l'assemblage minéralogique des roches métasomatisēes (métasomatisme I et II), nous observons le rempiacement graduel du quartz, de la biotite et des feldspaths alcalins par l'aégyrine et l'augmentation substantielle du volume de la phase exsolvêe des gros cristaux de microcline perthitique. Ces changements de l'assembiage minéralogique sont produits par les transferts de masse suivants: apport de fer $\left(6.4 \%\right.$ poids), $\mathrm{Na}_{2} \mathrm{O}(4.3 \%)$, MnO $(0.15 \%)$, Nb en quantité variable. L'aluminium est restée relativement stable, nous avons calculé une perte de $\mathrm{SiO}_{2}(3.5 \%), \mathrm{k}_{2} \mathrm{O}(2.1 \%)$, Rb et Ba en quantité variable. L'êtat d'oxidation du fer passe de $\mathrm{Fe}^{+}{ }^{+}$dans les roches non-mëtasomatisées à $\mathrm{Fe}^{+}+{ }^{+}$ dans les roches métasomatisées. 
Le métasomatisme IV a produit 1 'apport de $\mathrm{CaO}, \mathrm{P}_{2} \mathrm{O}_{5}, \mathrm{MgO}, \mathrm{TiO}_{2}$ et $\mathrm{Sr}$ (en quantité variable). Le métasomatisme III constitue la néphélinisation des enclaves de gneiss, observé dans la monzosyénite à néphéline aux abords de la zone de contact entre le complexe et l'encaissant. Tous ces évenements métasomatiques ont produit une augmentation de volume de 1'ordre de $5 \%$ du volume total de la roche. 


\section{INTRODUCTION}

\section{1) - Géologie générale}

Le complexe igné alcalin de Crevier est situé à $55 \mathrm{~km}$ au nord-est de la localité de Girardville dans les cantons Crevier et Lagorce, comté de Roberval, (figure 1). D'une superficie de $25 \mathrm{~km}^{2}$, 1e complexe s'est mis en place dans des séries gneissiques migmatitiques de la province géologique du Grenville, plus précisément dans le "Quebec gneiss segment" de la ceinture centrale gneissique (Wynne-Edwards, 1972). Cette subdivision de la province du Grenville, forme une mince bande parallèle au front du Grenville. Elle est caractērisée par la prédominence de gneiss quartzo-feldpathiques archéens et la présence, de façon discontinue, de métasédiments tels que les quartzites, les marbres et les paragneiss du supergroupe de Grenville, dont la déposition remonte à 17001200 m.a. (Wynne-Edwards, 1972). Ces roches ont subi plusieurs événements tectoniques (orogénies kénorienne, hudsonienne, elsonienne et grenvilienne) qui, sucessivement, ont imprimées divers patrons structuraux et niveaux de métamorphisme à ces roches. II ne subsiste maintenant que les structures de plissement orientées N.E. imprimées à la roche par 7 'orogénie grenvilienne (950 m.a.; Frith,1971), qui a oblitérée les effets des autres évênements tectoniques antérieurs. Le niveau de métamorphisme atteint est.du facies amphibolite supérieur à granulite avec sillimanite et à maints endroits; une fusion partielle des roches archéennes èt des métasédiments est observée.

L'activitê ignée au cours des diverses périodes orogéniques est très variée, mais elle est reliée à d'autres subdivisions de la province 


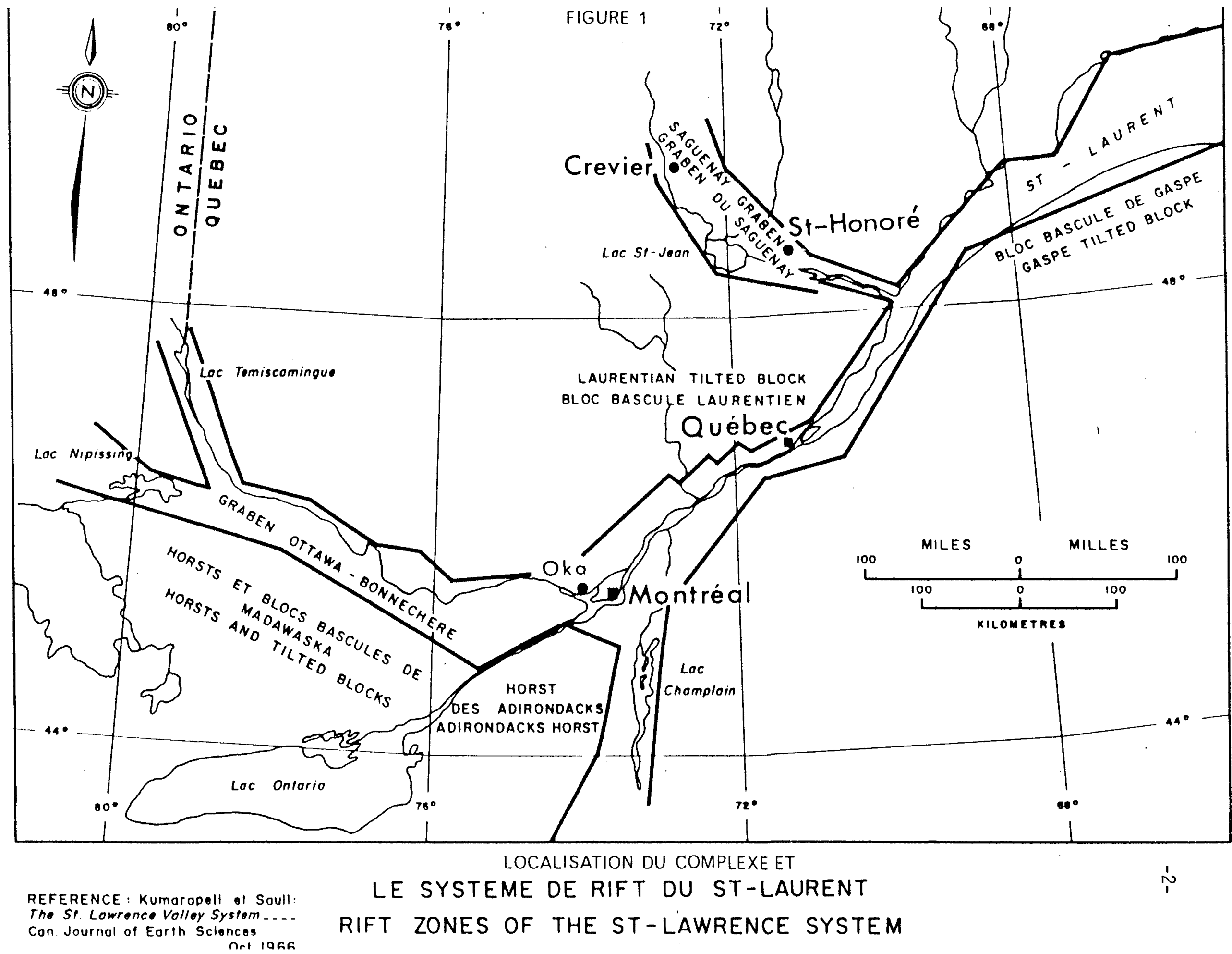


du Grenville, particulièrement les anorthosites et les gabbros du "Central granulite terrains", les intrusions alcalines de l'"Ontario gneiss segment" (syënites à néphêtine gneissiques de la rêgion de HaliburtonBancroft) et les intrusions granitiques en général, reliées à plusieurs périodes orogéniques et aux nombreuses subdivisions de la province du Grenville.

L'activité post-orogēnique est caractērisēe par des intrusions ignées du type syēnitiques, telles que les syênites alcalines à biotite de Mont-Laurier et du type sous-saturées telles que les complexes alcalins et carbonatites du reservoir Cabonga, d'Oka, de St-Honoré et les Montérégiennes qui sont peut-être reliés au graben du St-Laurent et son extension au Saguenay (Kumarape11i et Sal11, 1966). Mentionnons en dernier lieu, l'intrusion de dykes de carbonatite au nord du St-Laurent (parc Gatineau, Chicoutimi Nord) et enfin la déposition de sédiments paléozolques (shales et calcaires)

\section{2)- Travaux antérieurs}

Le complexe alcalin de Crevier a été découvert par la Soquem en 1975, à la suite d'un relevé radiométrique aéroporté. Depuis cette date, un grand nombre de travaux d'exploration ont été entrepris. Les principaux sont: un relevé scintillométrique, un relevé magnétométrique des relevés de polarisation provoquée, un relevé émanométrique, la cartographie détaillée du complexe et de l'encaissant et de nombreuses campagnes de forages.

Ces travaux ont permis de définir la forme du complexe, les différentes lithologies qui le compose, ses limites et la mise à jour 
d'indices minēralisés en tantale-niobium et uranium-niobium. (Aubertin, 1976; Bonneau, 1977; Bergeron et Laplante, 1978 et Bergeron, 1979)

3) - Méthodes de travajl et buts de la thèse

Le but de cette étude est de dêfinir la géologie, la pétrographie et la géochimie du complexe igné alcalin et de son encaissant métasomatisé.

Le complexe et son encaissant seront décrits du point de vue géologique et structural, à partir des travaux de prospection géologique et des données de forage. Du point de vue pétrographique et géochimique, à partir d'échantillions prélevés sur le terrain et dans les forages.

A la suite de ces travaux, l'auteur se propose de décrire et de définir les phases et les mécanismes qui sont intervenus lors de la mise en place des différents types de roches du complexe; la succession chronologique de ces évênements et les effets de l'intrusion sur l'encaissant.

Les résultats de ces travaux serviront, entre autre, à l'interprétation géologique des mombreux sondages implantēs sur la propriēté de la Soquem. Ils permettront une meilieure compréhension et interprétation des phases minéralisées qui accompagnent l'intrusion de certaines lithologies et enfin, ils donneront une bonne image du comportement de certains éléments lors du processus de métasomatisme inhërent à la plupart des intrusions du type de celle de Crevier 
CHAPITRE I

GEOLOGIE DU COMPLEXE DE CREVIER

Cette partie de l'étude du complexe traite de la nomenclature des différents types de roches, la minéralogie et les textures de leurs constituants, la succession chronologique des événements ignés, ainsi que la structure du massif.

\subsection{Pétrographie}

\section{1-1 Nomenclature}

La nomenclature des 1 ithologies du complexe alcalin de Crevier a été déterminée à partir de leur contenu en minéraux leucocrates (Tes feldspaths alcalins, les feldspathóides, les plagioclases et le quartz.) Le contenu a été calculé par analyse modale (tableau 1) au compteur de points. Les roches ont étê classifiées à partir de leur position sur le double triangle QAPF, proposé par Streckeisen (1976)

Les types de roches composant le complexe de Crevier sont:

(Fig. 2)

1) - Les monzosyénites (S)

2) - Les diorites à biotite (Db)

3) - Les monzosyénites à néphēline, comprenant:

a) - Les monzosyénites à néphēline I (SN I)

b) - Les monzosyénites à néphēline pegmatitique II (SN II) 
TABLEAU I

DES DIFFERENTS MINERAUX DETERMINES PAR

ANALYSE MINERALE AU COMPTEUR DE POINTS

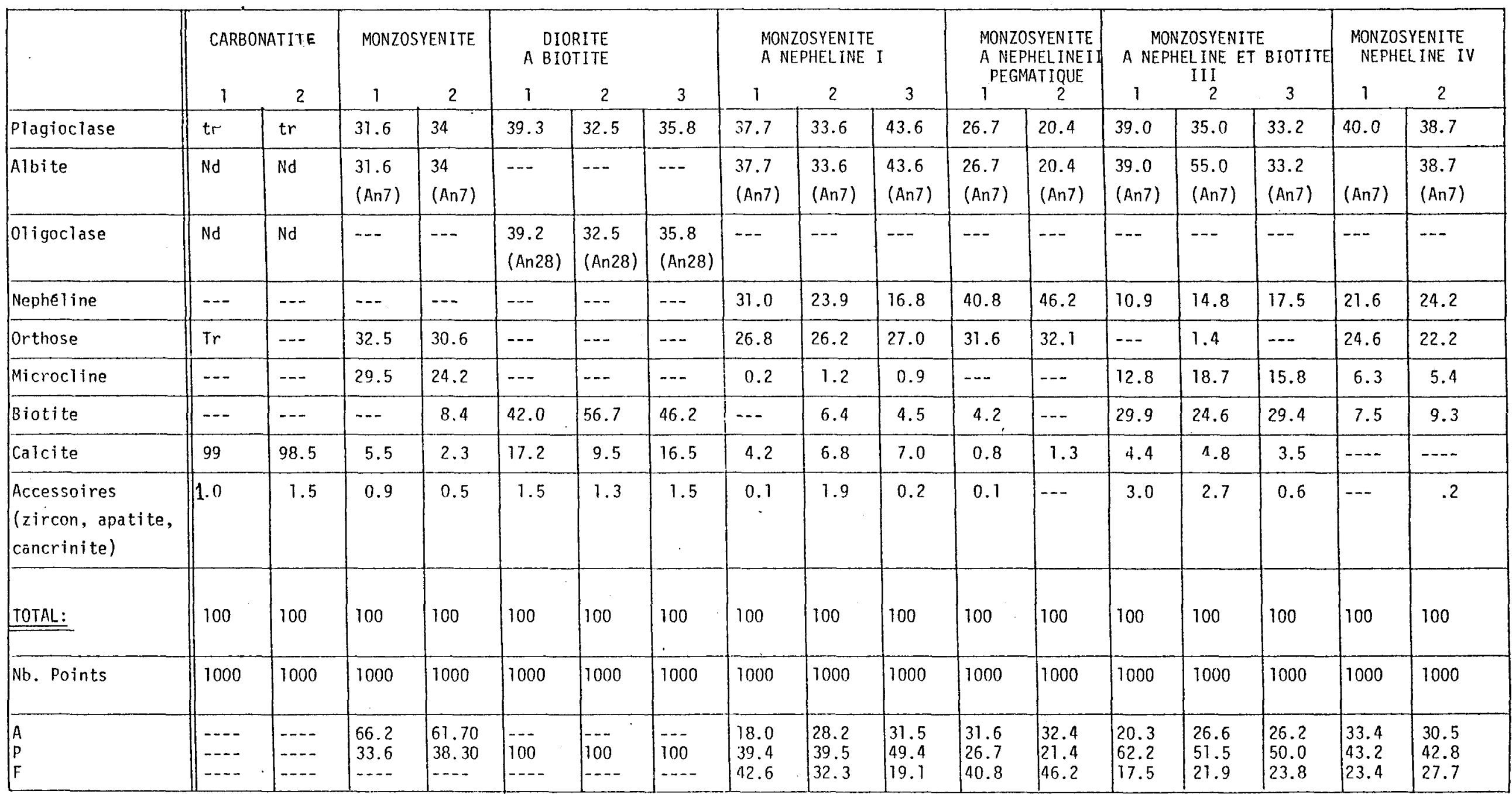

N.D. ---Non déterminé 
FIGURE 2

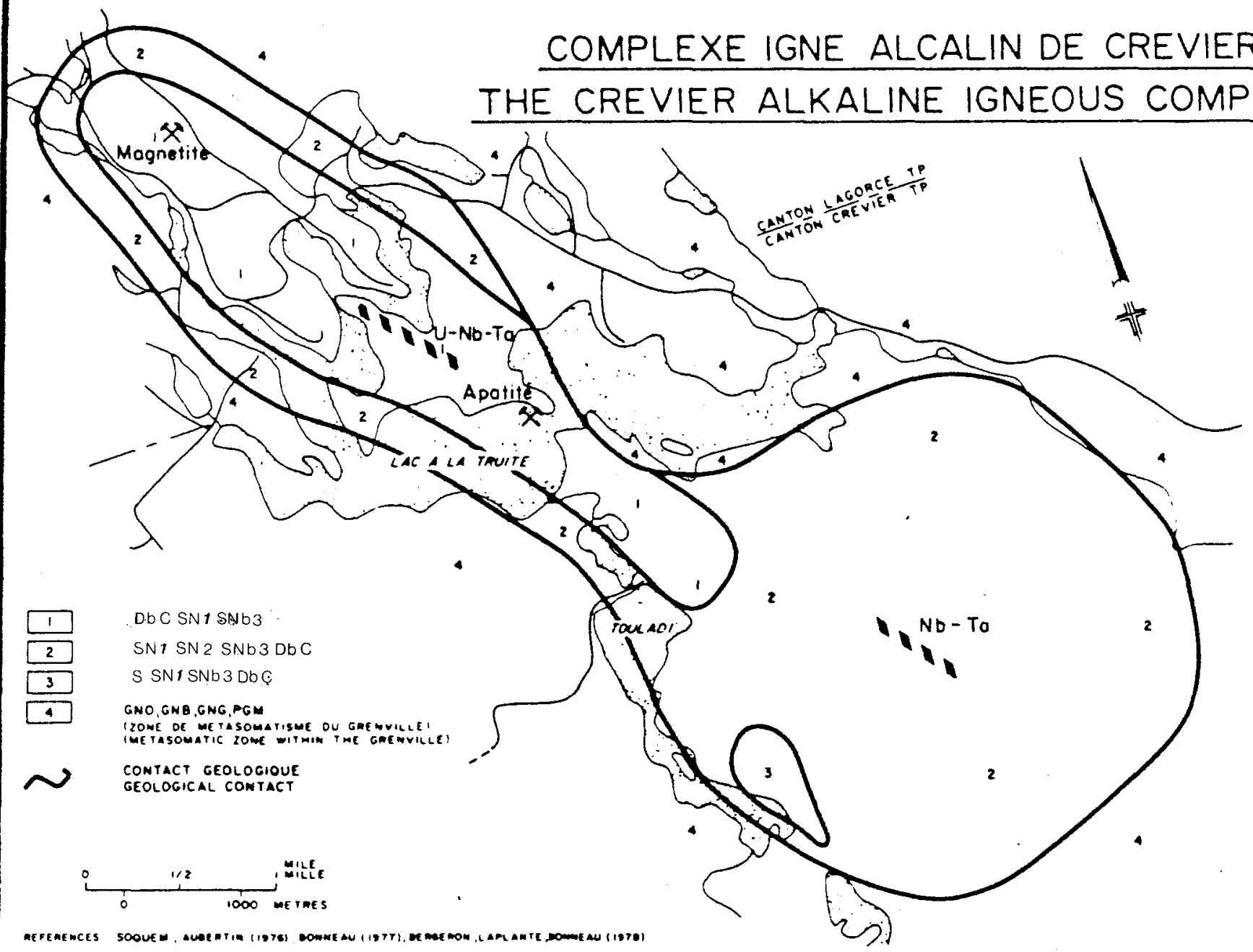


c) - Les monzosyénites à néphēline et biotite III (SNb III)

d) - Les monzosyénites à néphêline IV (SN IV)

4) - Les carbonatites

Notons que les monzosyénites à néphéline et les monzosyēnites, sont assez rapprochées du domaine des syênites à néphélines et des syênites. Le respect rigoureux de la classification de Streckeisen qui considère un plagioclase, où An<5, comme feldspath alcalin, nous ablige à considérer les plagioclases comme tels et non pas comme des feldspaths alcalins, bien que la calcité des plagioclases des roches du complexe est très faible (An 7). Des méthodes de détermination plus précises (microsonde) pourraient fort bien montrer une calcité plus faible et par le fait même changer la nomenclature des roches.

\section{$1.1-2$ Les monzosyênites}

Les monzosyénites ne composent qu'un faible pourcentage du volume de roches du complexe de Crevier. Nous en observons très peu sur le terrain et dans les forages. Leurs relations avec les autres types de roches du complexe sont par le fait même rarement observées.

La monzosyênite est une roche à grains fins ( 2 à $5 \mathrm{~mm}$ ) dont la couleur rose en surface frậche diffère peu de sa teinte en surface altêrée. La roche est composée de plagioclases, de feldspath alcalins, de calcite, de zircon, d'apatite et occasionnellement de la biotite et de l'aégyrine; elle possède une texture hypidiomorphe granulaire (planche I). Les plagioclases se répartissent en deux catégories, soit les plagioclases 1 (An 7) de forme tabulaire, leur tajlle varie de 0.2 à $2 \mathrm{~mm}$, les grains plus grossiers possèdent des mâcles de glissement ("glide twinning"; Smith, 1974). Les contacts sont nets avec les autres phases minéralogiques de 
la roche. Un certain nombre d'individus ( $8 \%$ ) contiennent des exsolutions d'orthose, disposées paralièlement aux mâcles du plagioclase. Le plagioclase II est une albite provenant de l'exsolution de l'orthose et du microcline. Ces derniers sont hypidiomorphes, de forme tabulaire, et leur taille varie de 0.5 à $2 \mathrm{~mm}$. La phase exsolvée est de type "en taches" pour le microcline et de type veine pour l'orthose. (Smith,1974) Sur certains individus la phase exsolvée sort du feldspath alcalin et forme une couronne d'albite autour de ce dernier allant même parfois s'immiscer dans les plagioclases I adjacents. L'orthose contient occasionnellement des inclusions de plagioclase I et elle est légèrement altérée en séricite. Le microcline contient des inclusions d'orthose et de plagioclase I.

La calcite est interstitielle dans 1 a roche sous forme de plages de 2 à $3 \mathrm{~mm}$. Elle ne semble pas altérer les feldspaths on observe au contact de ces derniers, une légère corrosion atténuant le contact entre la calcite et le grain attaqué. Les accessoires sont composés de zircon et d'apatite. Ils sont de forme sphérique dont le diamètre varie de 0.5 à $1 \mathrm{~mm}$. Ces minéraux sont interstitiels dans la roche.

L'examen de plusieurs échantitions a révêté la présence occasionnelle de biotite $(1-5 \%)$ et particulièrement un échantillon oú on a observé de l'aégyrine. La présence de ce minéral est accidentelle, car l'échantilion a été pris près de la zone de contact du massif adjacent à des gneiss aégyrinisés. 
L'ordre d'apparition des minéraux déduit des relations texturales est le suivant:

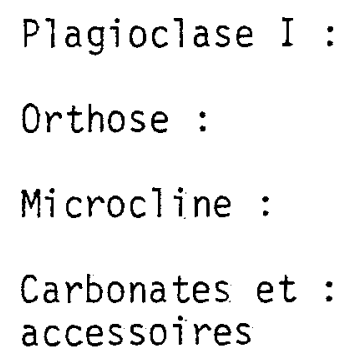

\section{1-3 Les_diorites_à bijotitte}

La diorite à biotite compose $15 \%$ à $20 \%$ des roches des différentes unités du complexe. Nous l'observons sous forme de bandes de $20 \mathrm{~cm}$ à $1 \mathrm{~m}$ (essaims de dykes parallètes) et de dykes tardifs de quelques centimètres de 1argeur. La roche est à grains fins, de couleur noire en surface fraîche et altérée. L'examen de lames minces à faible grossissement révèle un faible rubannement, formé par la concentration de biotite à grains grossiers. La roche est constituée de biotite, de plagioclases, de calcite, de zircon et d'apatite. (planche 2)

Les grains de biotite sont hypidiomorphes de forme tabulaire et 1 a dimension des grains varie de 0.5 a $2 \mathrm{~mm}$. Ils contiennent des inclusions de plagioclases, certains individus sont partiellement ou complètement remplacés par la calcite.

Les plagioclases forment deux groupes distincts de par leur:; texture. Le premier groupe identifié plagioclase 1 (An 20) est de forme tabulaire légèrement allongé et leur taille varie de 0.5 à $1 \mathrm{~mm}$. Leurs mâcles sont floues et les bordures de grains sont irrégulières, les grains 
sont interlobés. Ils contiennent un grand nombre d'inclusion de biotite xénomorphe. Le deuxième groupe, identifié plagioclase II (An 20), est de forme asciculaire et la taille des grains est de 1 a $2 \mathrm{~mm}$ dans leur grand axe. Ils forment des demi rosettes dans la roche et ils contiennent des inclusions de biotite.

La nature du contact entre les différents types de plagioclases est à noter. Les contacts entre les individus du même groupe sont nets, par contre celui entre les plagioclases I et II est flou, souvent indiscernable. Les différentes textures observées tendent à montrer que les plagioclases ont subi une légère recristallisation.

La calcite est interstitielle; elle forme des plages xénomorphes de 0.5 à $3 \mathrm{~mm}$. Certaines plages sont clairement identifiées comme des pseudomorphes de biotite, nous observons des grains de biotite partiellement et/ou complètement remplacés par la calcite.

Les accessoires, le zircon et l'apatite, sont disséminés dans la roche. Ils possèdent une forme sphérique et un diamētre moyen de 0.5 $\mathrm{mm}$.

Ce type de roche semble assez unique, car suite à une consultation de plusieurs ouvrages (Sorensen, 1971; Heinrich,1966; Currie,1976 et Johanssen, 1932) qui décrivent de nombreux complexes alcalins et occurrences de carbonatite, aucune des lithologies décrites ne correspondent à la diorite à biotite observée sur le terrain et dans les forages.

L'ordre d'apparition des minéraux déduit des observations texturales, est le suivant: 


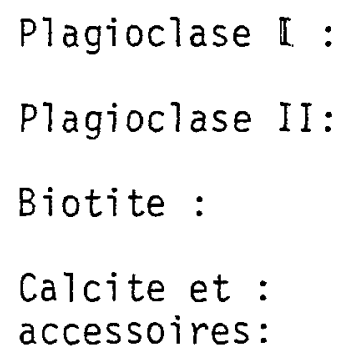

\section{1-4 Les monzosyénites à néphé] ine}

Les monzosyênites à néphêline composent la majorité des roches du complexe de Crevier. Nous les observons sous plusieurs formes sur le terrain et ces différentes formes sont reliêes à des événements précis dans leur succession chronologique. Pour ces raisons nous décrivons les différentes monzosyénites à néphêtine selon chaque occurrence.

a) - Les monzosyénites à néphētine I

La roche est de couleur blanchâtre avec une légère teinte rose en surface frâ̂che et blanche en surface altērée. Sur cette surface nous observons le relief négatif caractéristique que produit les cristaux de néphéline lessivés, par rapport aux autres constituants. La néphēline en cassure fraiche, est facile a identifier par sa couleur rose et son aspect vitreux. La roche est composée de plagioclases, de feldspaths alcalins, de néphéline, de cancrinite, de calcite, de zircon et d'apatite. La texture générale de la roche est hypidiomorphe granulaire. (planche 3)

Le plagioclase (An 7) est de forme rectangulaire et leur tailie varie de 3 à $4 \mathrm{~mm}$ suivant leur grand axe. Les grains montrent des mâcles de glissement et la majorité sont antiperthitiques, du type en taches. La phase exsolvêe (microcline) compose parfois jusqu'à 50\% de l'antiperthite. Elle peut migrer à l'extérieur du plagioclase et forme des grains xénomorphes de $0.2 \mathrm{~mm}$ de diamètre autour de ce dernier. Le feldspath alcalin est 
une orthose perthitique et la taille des grains varie de 4 a $6 \mathrm{~mm}$. La phase exsolvée est du type veine, occasionnellement elle migre hors de l'orthose et forme une couronne partielle d'albite autour du grain. Cette couronne d'albite, au contact avec un plagioclase, pénètre à que]ques endroits dans ce dernier et efface le contact net entre les deux minéraux. Par contre, la phase exsolvée du plagioclase antiperthitique qui migre à l'extérieur du grain ne s'immisce pas dans une orthose perthitique qui serait en contact.

La néphêline apparait comme phase interstitielle. Elle se présente sous forme de grains libres, de forme pseudo-circulaire et leur taille varie de 1 a $3 \mathrm{~mm}$. Elle possède des contacts nets avec les autres phases minérales et elle est logée à travers les minéraux épousant leur forme.

La calcite, l'apatite, le zircon et la cancrinite composent un assemblage minéralogique secondaire. La calcite se présente sous forme de plages xénomorphes de 1 à $4 \mathrm{~mm}$ et elle est interstitielle dans 1 a roche. L'apatite et le zircon sont xénomorphes et leur taille varie de 0.5 a $1 \mathrm{~mm}$. Ils sont interstitiels dans la roche. La cancrinite est le résultat de l'altération de la néphéline par la calcite. Nous observons des couronnes de cancrinite autour des grains de néphéline en contact avec la calcite (planche 4). 
L'ordre d'apparition des minéraux, déduit des observations texturales, est le suivant:

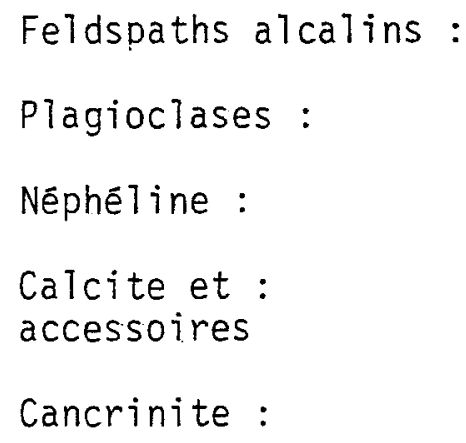

b) - Les monzosyénites à néphēline pegmatitiques II

Ce facies est caractérisé par la concentration de phénocristaux de nêphêline de couleur rouge foncé dans la monzosyēnite à néphêline I. Ils sont de forme rectangulaire et leur taille varie de 1 à $4 \mathrm{~cm}$; la concentration des phēnocristaux forme des poches et/ou des dykes diformes de quelques centimètres à un mètre de largeur en général, qui exhibent des contacts diffus avec la monzosyénite à néphéline I (planche 5). Un de ces dykes constitue la zone minéralisée en tantale-niobium, mise à. jour par les forages. L'examen microscopique. de ce type de roche nous a montré une composition et des textures identiques à la monzosyénite à néphéline I. Les phénocristaux de néphēline contiennent de nombreuses inclusions de plagioclases antiperthitiques et ils sont altérés en cancrinite, principalement dans les fractures du grain. Le contact des phénocristaux avec les autres phases minérales est net mais irrégulier (planche 6), nous observons parfois des inclusions de néphéline dans les minéraux adjacents, ces inclusions ont une extinction commune avec le phénocristal. Il s'agit probablement d'imbrication de ce dernier dans le minéral en contact. 
La présence de phēnocristaux de néphéline (SN II) dans la monzosyénite à néphéline I peut être expliquée par la cristallisation tardive d'un magma riche en volatils, suite à l'intrusion de la monzosyénite à néphéline. I. On peut faire l'analogie aux pegmatites qui cristallisent tardivement suite à la mise en place d'un magma granitique (Menhert, 1968).

La roche présente les mêmes phases minérales secondaires décrites dans la roche précédente.

L'ordre d'apparition des minéraux, déduit des observations texturales, est le suivant:

Feldspath alcalins:

Plagioclase:

Nêphéline:

Phênocristaux de:

nêphēline

Calcite et:

accessoires

Cancrinite:

c) - Monzosyênites à néphéline et biotite III

La monzosyénite à néphéline et biotite III possède les mêmes caractéristiques minéralogiques et texturales que la monzosyēnite à nêphêline I. Les particularités concernant ce type de roche sont: 1) le pourcentage des constituants (voir tableau 1), 2) la présence de biotite, 3) la phase exsolvée des plagioclases antiperthitique est de l'orthose plutôt que du microcline. 
La biotite est de forme tabulaire et la taille des grains varie de 0.5 a $3 \mathrm{~mm}$. Ils contiennent des inclusions d'orthose et les contacts sont nets avec les autres phases minérales.

L'ordre d'apparition des minéraux, déduit des observations texturales, est le suivant:

Feldspath al,calins:

Plagioclases:

Biotite:

Nêphêline:

Calcite et:

accessoires

Cancrinite:

d) - Les monzosyénites à néphéline IV, dykes tardifs.

Les monzosyēnites à nēphēline IV sont intrusives sous forme de dykes, à travers toutes les lithologies du complexe ainsi que dans l'encaissement métasomatisé. Les dykes ont de 10 à $50 \mathrm{~cm}$ de largeur, ils sont à grains fins de couleur gris foncé en surface altérée et gris pâle en surface fraîche. Une certaine variētē de ces dykes montre des textures particulières. Nous y observons la concentration de grains de néphéline altérée, en bordure du contact du dyke. Les grains de néphéline sont de couleur blanche et leur taille est de $0.5 \mathrm{~cm}$. Cette variēté de dykes n'est observée que dans l'encaissant du complexe. La roche est composée de plagioclases de feldspaths alcalins, de néphêline, de biotite, de zircon, d'apatite et de calcite. 
Les plagioclases (An 7) sont de forme tabulaire et leur taille varie de 1 a $3 \mathrm{~mm}$. Ils sont caractérisés par leur texture poéçilitique, occasionnée par la présence de néphéline de forme sphérique dont la taille est de $0.2 \mathrm{~mm}$. Certaines inclusions ont une forme de crochet similaire aux textures de simplectites que l'on peut observer dans les granites, entre le quartz et le plagioclase. Le contact avec les inclusions de nēphéline est flou, à fort grossissement nous observons des textures de flamme de la néphéline dans l'albite. (planche 7)

La néphéline est aussi observêe sous forme de grains interstitiels dans la roche et leur taille varie de 2 à $4 \mathrm{~mm}$. Elle contient de nombreuses inclusions de biotite, de feldspaths alcalins et de plagioclases. Les inclusions de certains grains sont orientées, elles forment un patron hexagonal dans la néphéline. Cette dernière est légèrement altērée en paragonite.

Les feldspaths alcalins sont composés d'orthose perthitique de type veine et de microcline. L'orthose est tabulaire, sa taille varie de 1 à $4 \mathrm{~mm}$ et elle contient de nombreuses inclusions de biotite. Les grains de microcline sont idiomorphes de forme carrée et leur taille est de $0.5 \mathrm{~mm}$. Ils sont frais et ne contiennent aucune inclusion.

La biotite est de forme tabulaire et la taille des grains varie de 0.2 à $2 \mathrm{~mm}$. Ils ne contiennent aucune inclusion et sont disposés en grains Tibres dans 1 a roche. Leur bordure est souvent corrodée, formant une mince pellicule au contact avec les autres grains.

La phase minérale secondaire est composēe de calcite, de zircon, d'apatite, d'opaques et de cancrinite provenant de l'altération de la néphêtine par la calcite. Le zircon, l'apatite et les opaques sont xénomor- 
phes, interstitiels dans la roche et leur taille varie de 0.5 a $1 \mathrm{~mm}$. L'examen microscopique de la deuxième variété de dykes nous montre un assemblage minéralogique ét des textures similaire à la première variétē. La nature du contact avec l'encaissant présente certaines particularités, nous observons une zone de transition de quelques millimètres d'épaisseur, entre l'encaissant et la concentration de grains de néphêline. Cette zone est complètement dépourvue de plagioclases et elle ne contient que des grains fins de microcline et d'orthose.

Les relations décrites entre la néphéline et les plagioclases, impliquent que la cristallisation de la néphéline s'est faite aux dépends de l'albite.

L'ordre d'apparition des minéraux, déduit des observations texturales, est le suivant:

Feldspaths alcalins:

Plagioclases:

Néphêline:

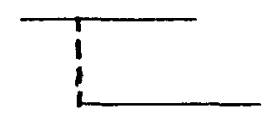

Biotite:

Calcite et: accessoires

Cancrinite:

\section{$1.7-5$ La carbonatite}

La carbonatite du complexe de Crevier est assez homogène dans son ensemble. C'est une roche grenue, de couleur brun pâle en surface altérēe et blanche en surface fraîche. Elle est composêe essentiellement de 
calcite, formant des cristaux rhomboédrique de 0.5 à $2 \mathrm{~cm}$ d'arète. Les contacts entre les grains sont nets et rectilignes. La carbonatite contient de nombreuses inclusions de forme sphérique de plagioclases, de feldspaths alcalins et de biotite partiellement transformée en muscovite. Certains groupes $d$ 'inclusions montrent une extinction commune, ce qui inddique que les inclusions sont des restants de minéraux qui ont été digêrés par la calcite, lors de sa mise en place (planche 8). La carbonatite contient de l'apatite et du zircon disséminés dans la roche, en quantité variable (jusqu'a $15 \%$ et $2.5 \%$ respectivement dans certains sondages). Les mâcles lamellaires de quelques grains sont légèrement déformés, montrant une faible courbure.

Trois hypothèses furent proposées pour définir l'origine des carbonatites. La première, par Daly (1933), propose la formation des carbonatites par l'assimilation de calcaires par un magma alcalin. La deuxième hypothèse, soumise par Bowen (1958), propose une origine hydrothermale comme processus de formation. Enfin le troisième modèle est proposé suite aux travaux de Wyllie et Tuttle (1960) et Wyllie et Boettcher (1969) sur le système $\mathrm{CaO}-\mathrm{CO}_{2}-\mathrm{H}_{2} \mathrm{O}$. Ces travaux concluent à une origine magmatique pour ce type de roche. Les textures observées lors des expériences de Wyllie et Tuttle (1960) et celles rapportées par Gittins (1973), sont principalement la texture dendritique de la calcite ainsi que les textures porphyriques et trachytiques du produit de cristallisation. Ces textures sont fréquemment observées dans les carbonatites d'Aino (Sorensen, 1971) dans celles d'Oka (Girault,1966) et dans les carbonatites du Colorado (Heinrich, 1966). La granulométrie de la carbonatite du complexe de Crevier ainsi que ses textures, sont peu communes en regard des textures magmatiques ob- 


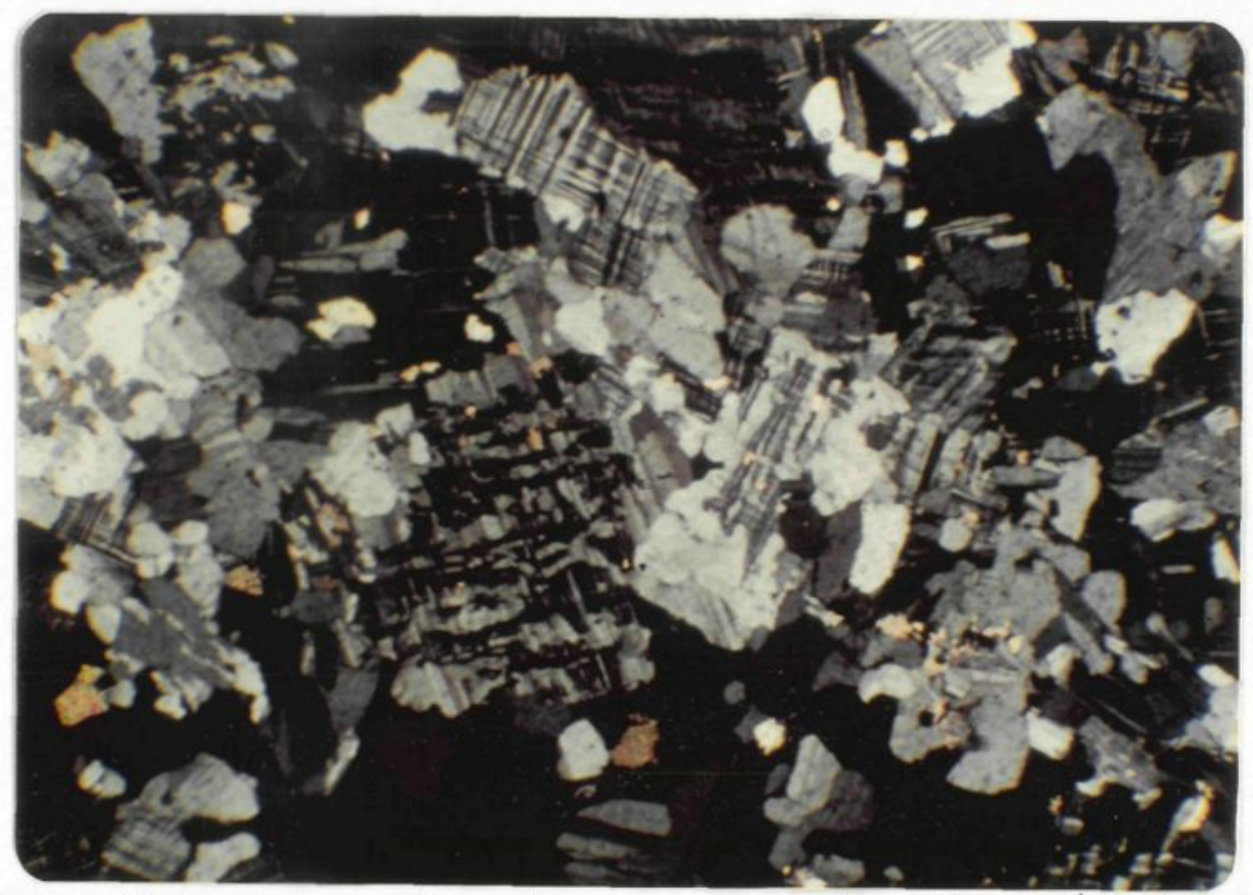

Planche 1: Microphotographie illustrant la minéralogie (feldspaths alcalins et plagioclases) et la texture hypidiomorphe granulaire des monzosyēnites. $\varnothing=6.5 \mathrm{~mm}$

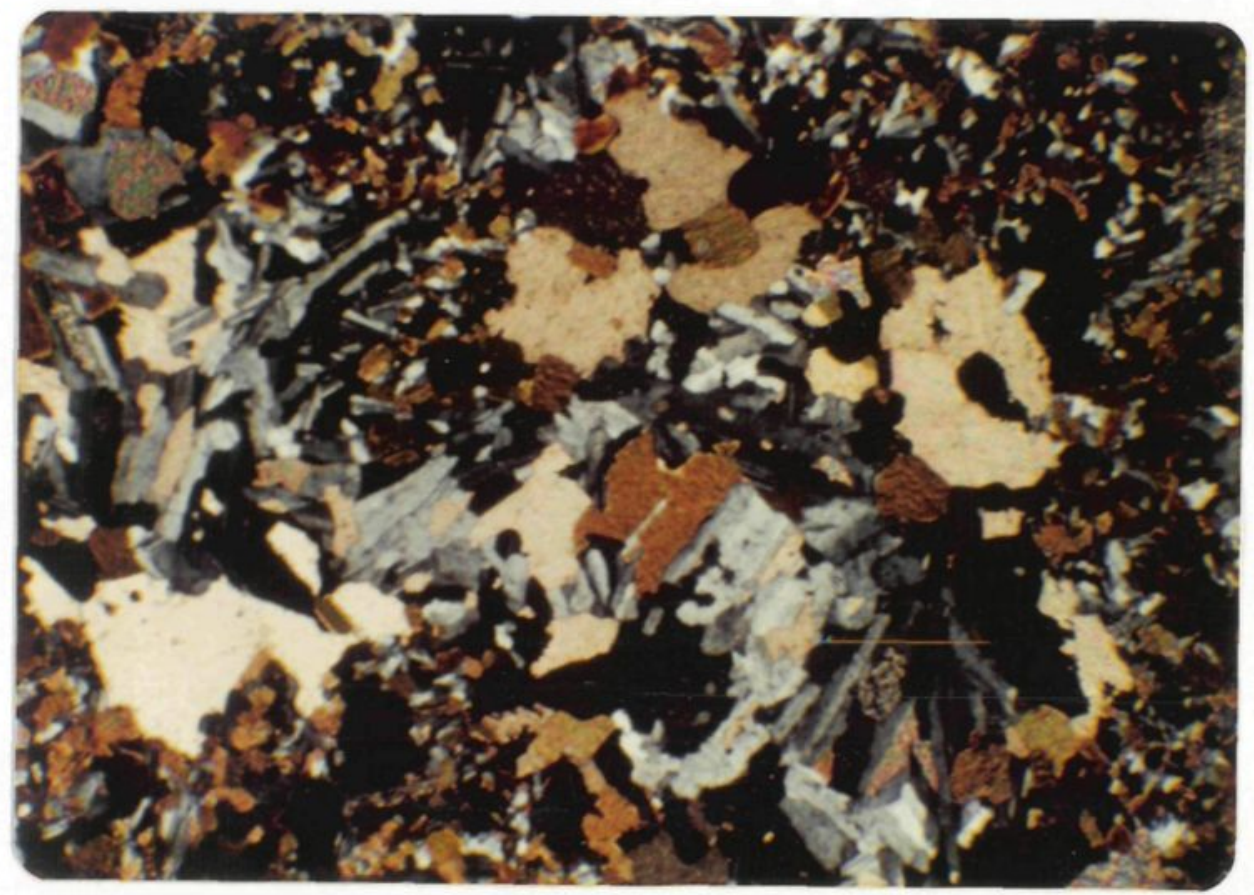

Planche 2: Microphotographie illustrant la minéralogie et la texture générale des diorites à biotite. Nous observons les plagioclases tabulaires I et les plagioclases xénomorphes II, possédant des contacts interlobés avec la biotite. $\phi=6.5 \mathrm{~mm}$ 


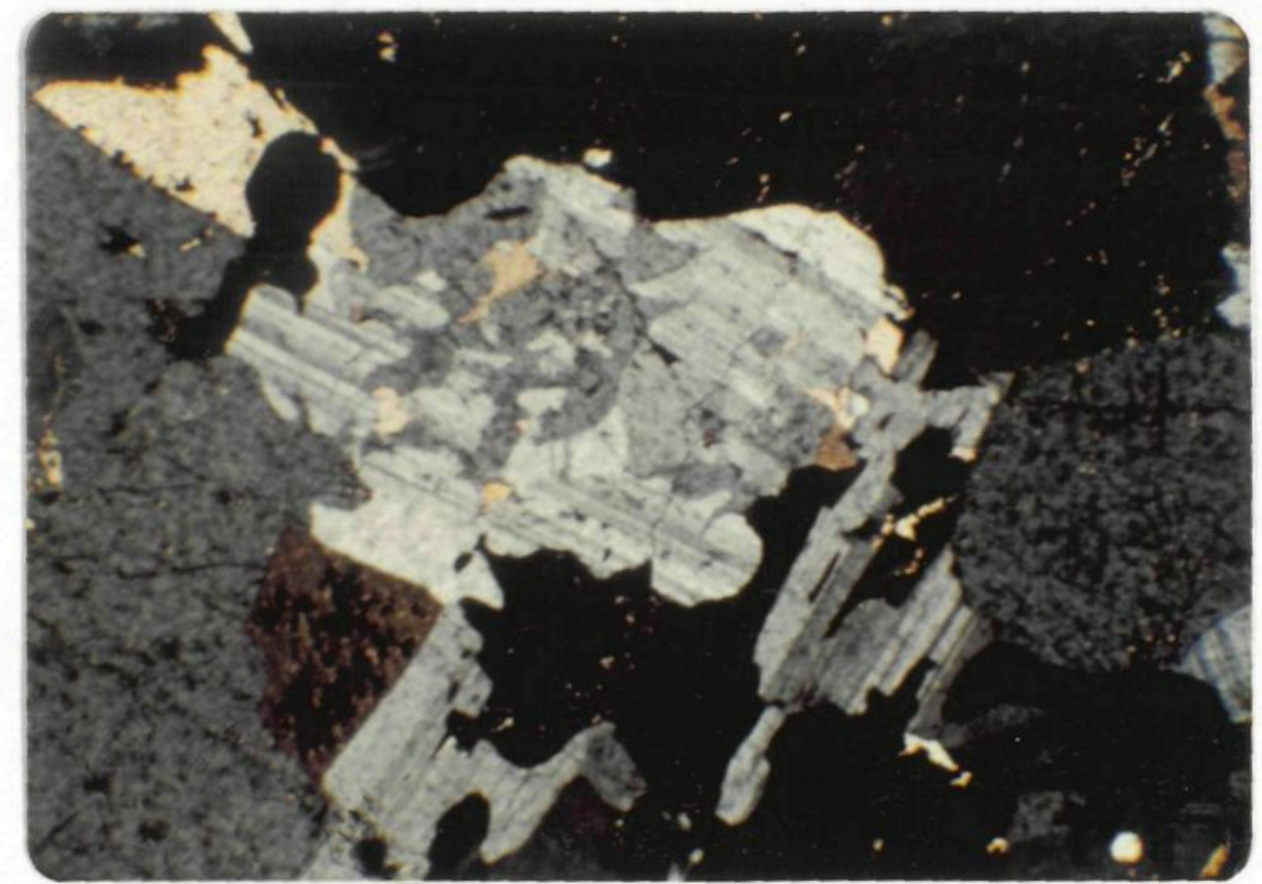

Planche 3: Microphotographie illustrant la texture hypidiomorphe granulaire des monzosyénites ã néphêline I, ainsi que la nature interlobée de la néphéline (minéraux foncés) entre les plagioclases antiperthitiques. $\phi=6.5 \mathrm{~mm}$.

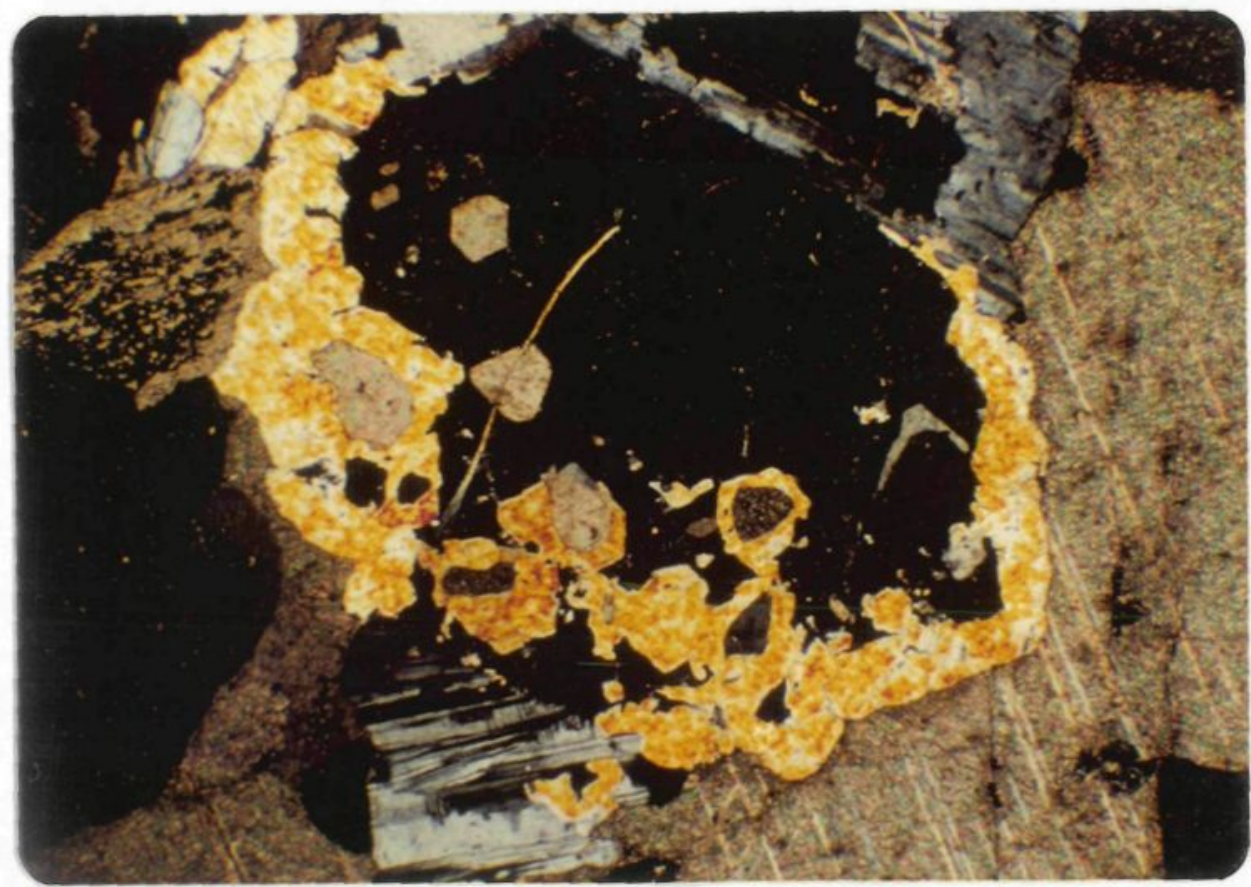

Planche 4: Microphotographie illustrant 1'altération caractêristique de la néphêline en cancrinite (couronne jaune) au contact de la calcite. $\phi=6.5 \mathrm{~mm}$. 


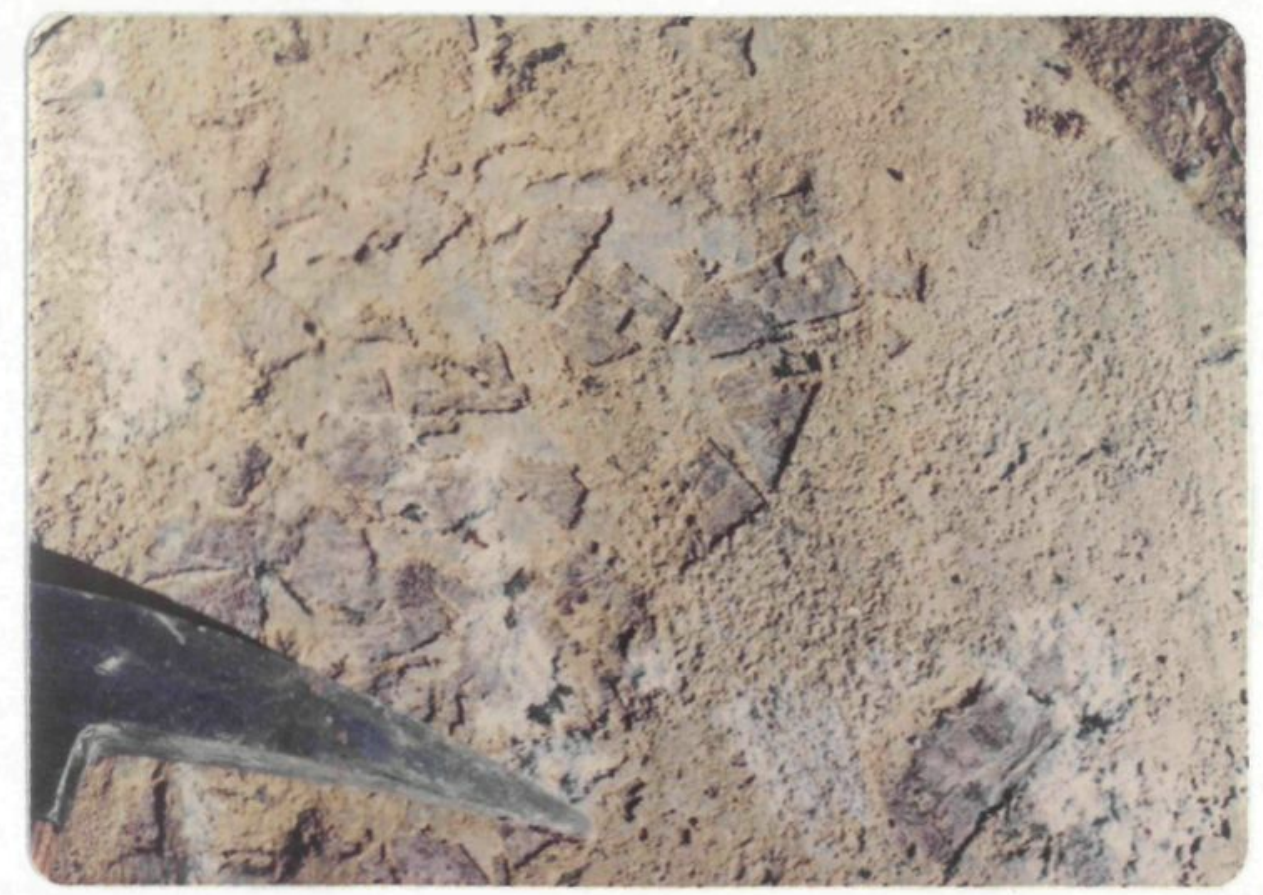

Planche 5: Photographie illustrant la texture mégascopique des dykes et/ou poches de monzosyēnites à néphēline pegmatitiques II dans la monzosyénite à néphéline I

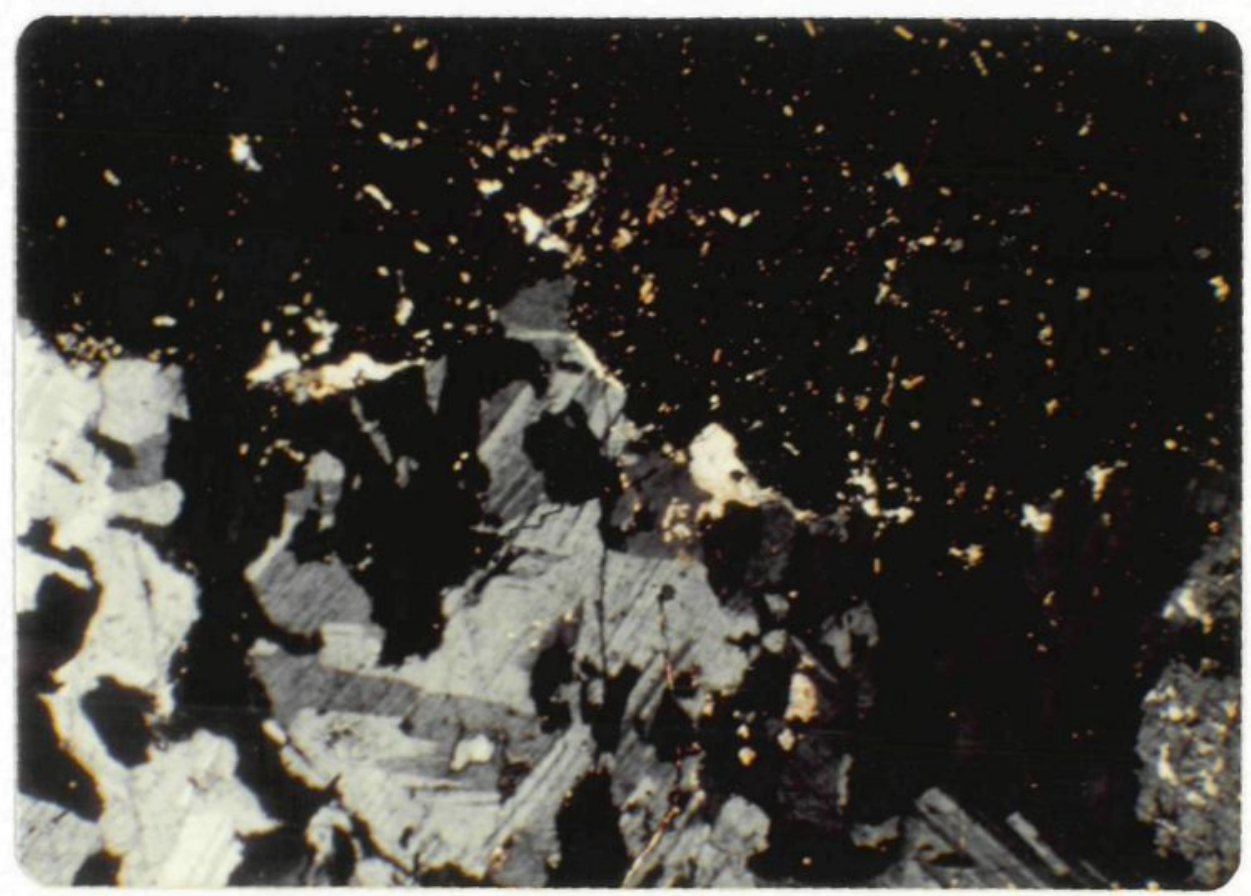

Planche 6: Microphotographie illustrant un gros cristal de néphéline d'une monzosyênite à néphêline pegmatitique II et ses contacts irréguliers avec le plagioclase antiperthitique. $\not=6.5 \mathrm{~mm}$ 


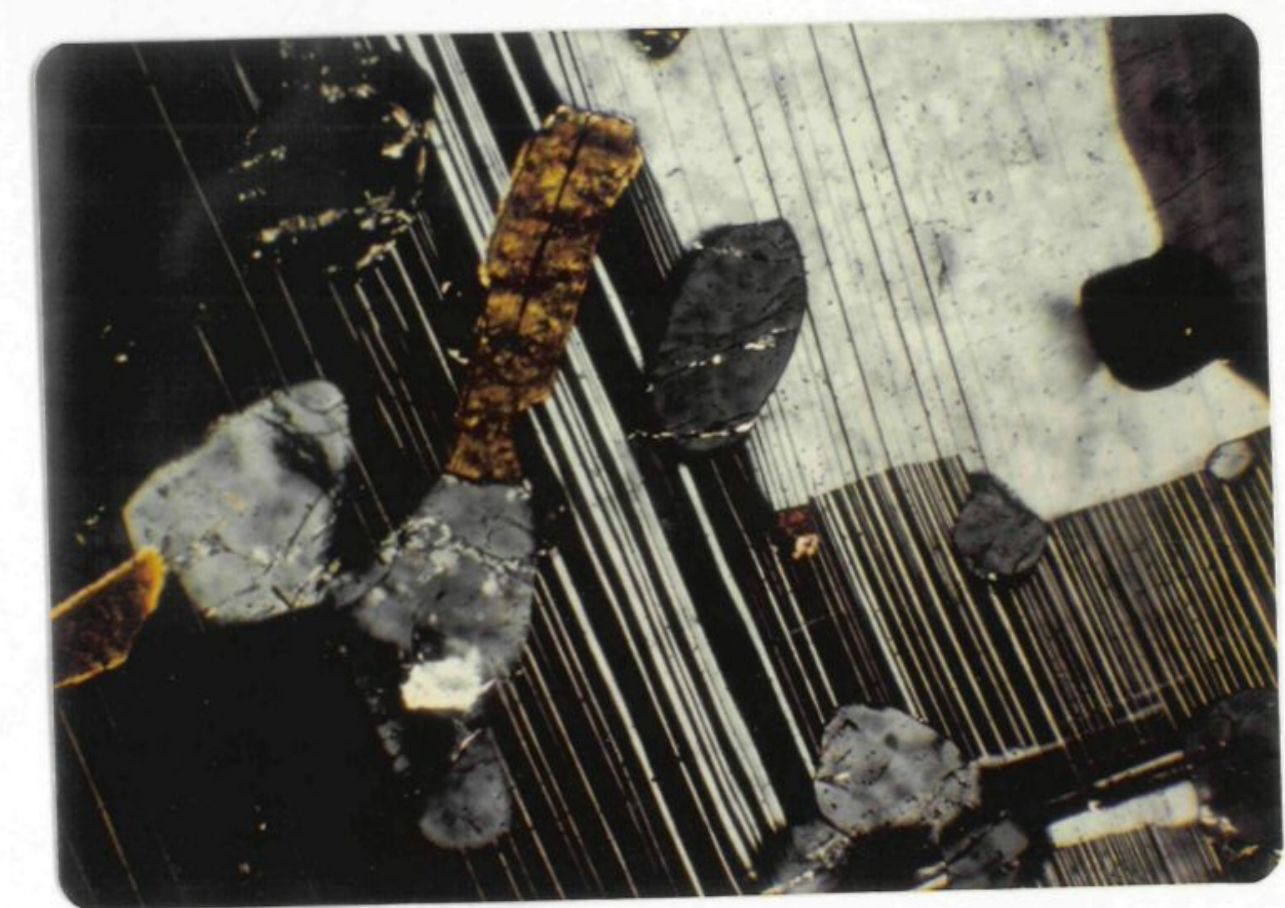

Planche 7: Microphotographie illustrant la texture poéçilitique du plagioclase dans les dykes tardifs de monzosyénite à néphêline IV et le contact diffus des inclusions de néphéline dans 1 'albite. $\varnothing=2.5 \mathrm{~mm}$

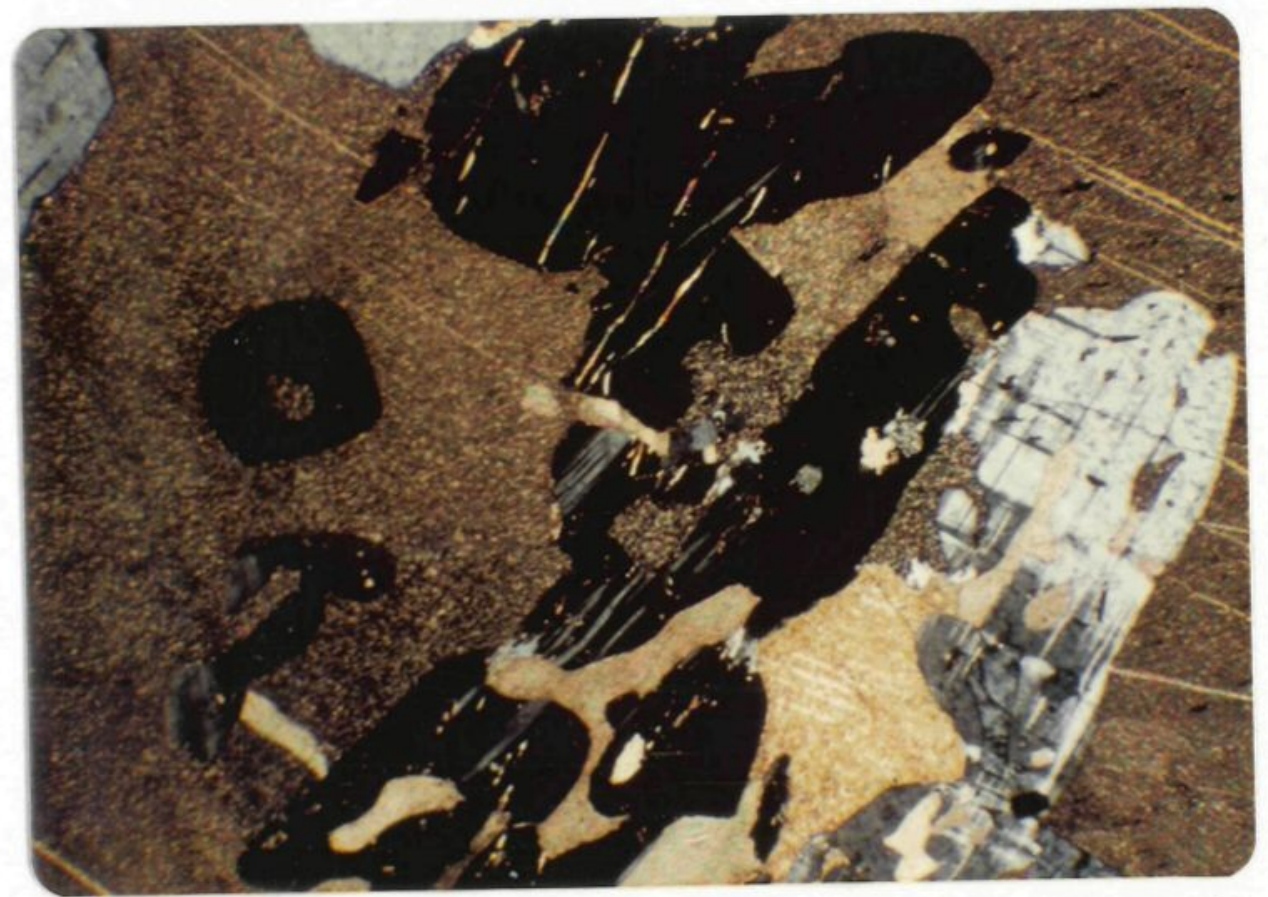

Planche 8: Microphotographie illustrant les plagioclases et les feldspaths alcalins partiellement digérés par la carbonatite. $\varnothing=6.5 \mathrm{~mm}$. 
servées par ces auteurs. Parsons (1961) décrit des carbonatites à grains grossiers, observẻes dans lè complexe de Firesand en Ontario, similaires à celles de Crevier.

Nous pouvons proposer 1 'hypothèse d'une origine hydrothermale pour la carbonatite de Crevier. Des études sur les inclusions fluides seraient necessaires pour lever le doute.

\subsection{Unités et structure du complexe de Crevier}

Le complexe alcalin de Crevier est composé de quatre grandes unités (figure 2), définies lors de la cartographie et des campagnes de forage. Les unités sont définies à partir de l'abondance relative des différentes lithologies décrites précédemment, et nous les décrivons suc: cessivement.

\section{2-1 Unitế.}

L'unité 1 forme la majorité nord du complexe. D'une superficie de $12 \mathrm{~km}^{2}$ elle est de forme allongée et elle possède une orientation génêrale de $320^{\circ}$. L'unité regroupe les lithologies suivantes:

- Carbonatite

- Diorite à biotite $26 \%$

- Monzosyénite à néphêline $\mathbb{I}$ $20 \%$

- Monzosyénite à néphêline et biotite II

- Monzosyénite 


\section{FIGURE 3}

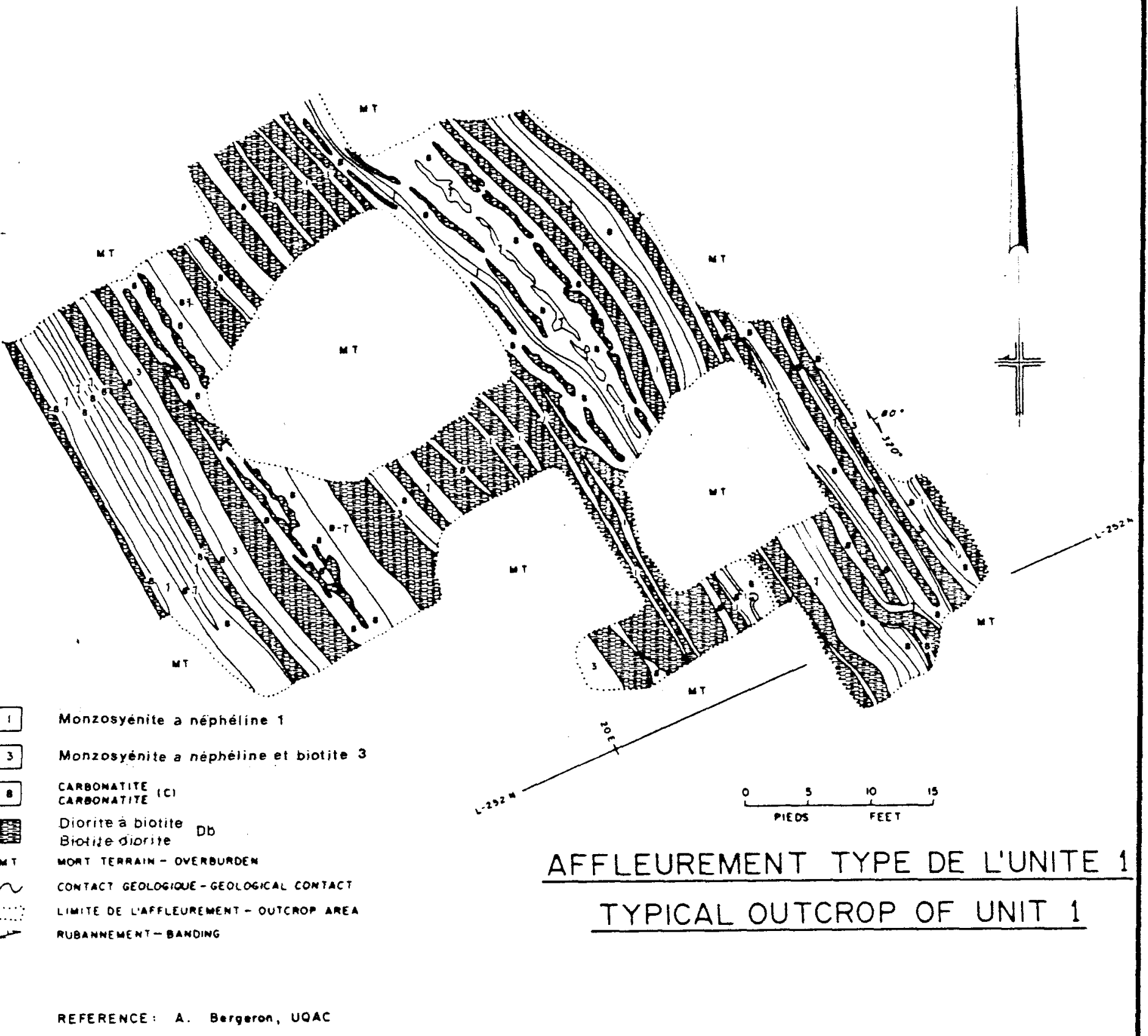


La caractéristique principale de cette unité est le rubannement décimétrique à métrique de la diorite à biotite, des monzosyénites à néphéline I et II et de la carbonatite. Ce rubannement forme des essaims de dykes paralièles, apparentés à des structures stromatiques (Mehnert,1968), orientês $300^{\circ}-340^{\circ}$ et un pendage de $70^{\circ}-90^{\circ}$ NE (figure 3 ). Les contacts entre les différents types de roches sont francs. Nous avons observé des dykes tardifs de monzosyénite à néphéline IV et de diorite à biotite recoupant les lithologies de l'unité, ainsi que des textures de fluage assez spectaculaires de SN I, Db et $C$. Notons que certaines bandes de monzosyēnite à néphēline I possèdent des textures particuliēres. Nous observons des phénocristaux de néphētine et d'orthose disposês perpendiculairement en bordure de la bande de SN I, au contact avec la diorite à biotite (planche 9). L'aspect et la disposition des phénocristaux impliquent .

que leur cristaliisation s'est faite sans contraintes et peut s'expliquer par une mise en place dans une zone d'extension.

C'est dans cette unité que nous avons délimité une zone porteuse de bétafite, minéral composé d'uranium, de niobium et de tantale (figure 2). Cette zone, d'une longueur de $900 \mathrm{~m}$ et large de $70 \mathrm{~m}$, est paralièle à la direction générale des roches du secteur. Les sulfures et oxides observés, en plus de la bétafite, sont: la pyrite, l'ilménite, la magnétite, le pyrochiore, le zircon et la chalcopyrite. Leur mode d'occurrence est sous forme disséminée et parfois en veinules millimétriques. La minéralisation est associée à la carbonatite. 


\section{2-2 L'unitêe?}

L'unité 2 est observée à deux endroits sur le complexe, soit une bande de $300 \mathrm{~m}$ de largeur, ceinturant en partie l'unité I et au sud de la propriété, une masse de forme circulaire de $12 \mathrm{~km}^{2}$ (figure 2).

L'unitê regroupe les lithologies suivantes:

- Monzosyênite à néphēline I et II

- Diorite à biotite $74 \%$

- Monzosyénite à néphēline et biotite III

- Carbonatite

Les différents types de roche qui composent cette unitê ne comportent pas d'épéments orientés. Nous observons une texture de bréchification omniprésente, qui provient de la mise en place de la monzosyénite à néphéline et biotite sous forme d'essaims de dykes non orientés, dans la monzosyénite à néphétine I (figure 4). Ces dykes ont une puissance de quelques centimètres à un mètre, les contacts avec la monzosyénite à néphéline I sont francs. La texture "bréchique" est similaire à la structure agmatique, décrite par Mehnert (1968) relative aux migmatites. La monzosyénite à néphéline I forme des blocs de $1 \mathrm{~m}$ à $5 \mathrm{~m}$.

En bordure de la zone de contact avec l'encaissant, nous observons des enclaves de gneiss oeillés aégyrinisés, complètement digērés par la monzosyénite à néphétine I. Ces enclaves, bien que détachées de l'encaissant, n'ont pas subi de rotation; leur foliation est la même que celle mesurée dans les roches de l'encaissant. 
Les forages ont intercepté dans la partie centrale du massif, une zone porteuse de tantale-niobium (figure 2). La minéralisation est sous forme de pyrochlore et est associée à la monzosyênite à néphéline pegmatitique II. La zone minéralisēe à une longueur de $200 \mathrm{~m}$ et est large de $30 \mathrm{~m}$. Elle constitue le seul élément orienté de l'unité 2, l'orientation est similaire $\left(320^{\circ}\right)$ aux lithologies et à la zone uranifère de T'unitê 1 .

Les principaux sulfures et oxydes observés dans les différents types de roches de cette unité sont: la magnétite, la pyrite, la pyrrohtine la bétafite et la chalcopyrite. Ces minéraux sont sous forme disséminée.

\section{2-3 L'unité 3}

L'unité 3 est șituée dans la partie sud-ouest du complexe (figure 2), de très petite taille elle a été délimitée principalement à partir de deux sondages. Les principaux types de roches qui la compose sont:

- Monzosyēnite à néphéline I $35 \%$

- Monzosyēnite $32 \%$

- Diorite à biotite $23 \%$

- Monzosyēnite à néphēline et biotite III $8 \%$

- Carbonatite 2\%

Aucune minéralisation d'importance n'a été observée dans cette unité. 
FIGURE 4
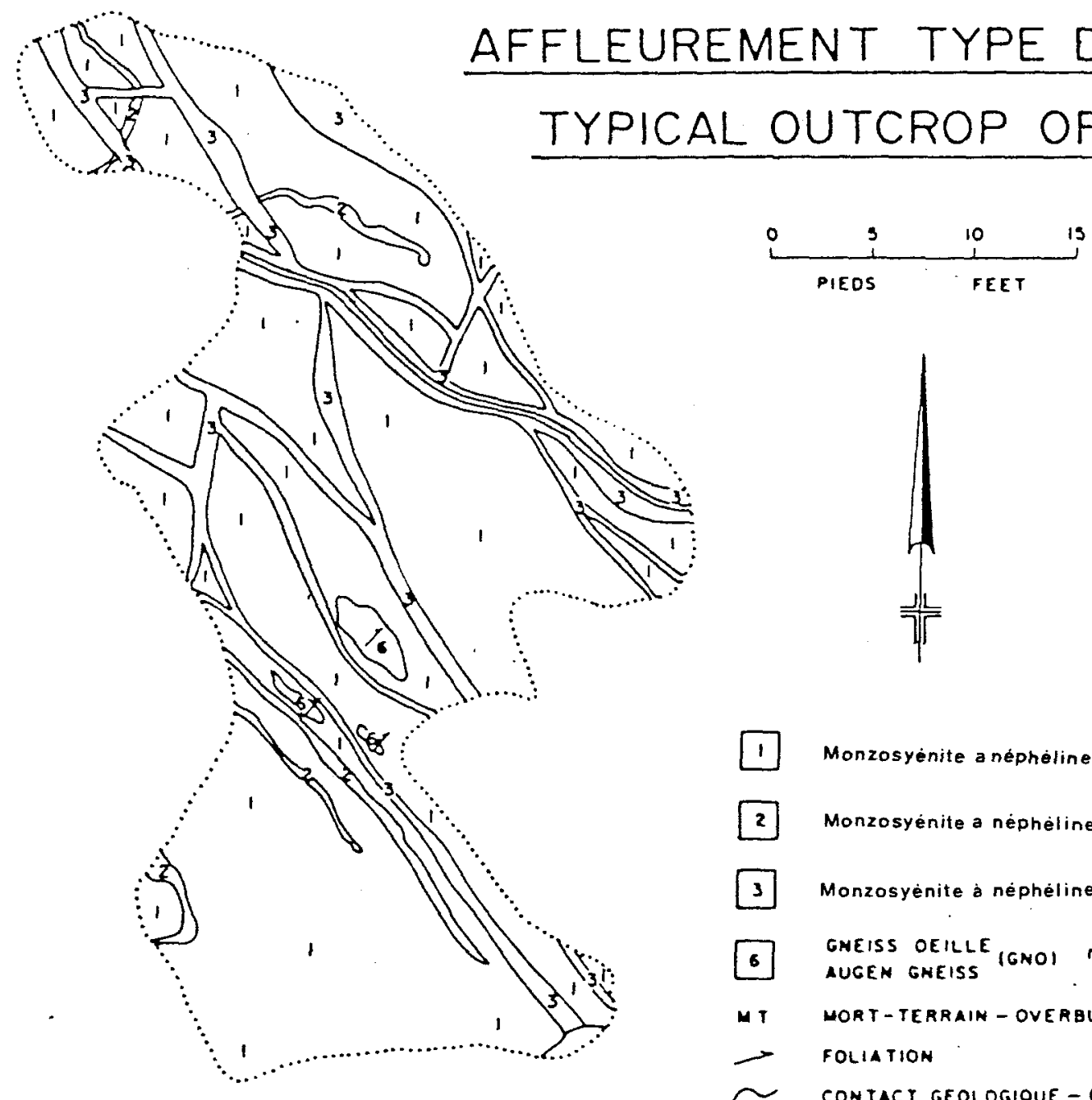

1 Monzosyènite a néphèline 1

2 Monzosyénite a néphéline pegmatitique 2

3 Monzosyenite à néphéline et biotite 3

GNEISS OEILLE (GNOI métasomatisé AUGEN GNEISS (GNO)

M T MORT - TERRAIN - OVERBURDEN

- Foliation

〜 contact geologioue - geological contact

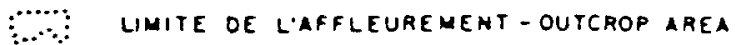

REFERENCE, A. Bergeron, UOAC 


\section{2-4 L'unité 4}

L'unité 4 regroupe quelques types de roches du Grenville observées et définies comme appartenant à l'encaissant, qui a été métasomatisé lors de la mise en place du complexe de Crevier. Les principaux types de roches de cette unitê sont:

- Les gneiss oejllés

- Les gneiss quartzo-feldspathique à biotite et hornblende

- Les amphibolites

- Les quartzites

- Les pegmatites

La description de la minéralogie et des textures de ces roches, fera l'objet d'un chapitre subséquent.

Le seut élément structural' inhérent à l'ensemble du complexe, est la présence d'un linéament majeur recoupant le complexe dans toute sa longueur. Le linéament est orienté $320^{\circ}$, il est marqué par 1 'alignement des bordures de lacs et des petits ruisseaux du secteur. Les zones minéralisées, mises à jour à l'aide des forages, sont situées en bordure de ce linéament. Un forage l'a traversé, et lors de l'examen des carottes aucun indice indiquant la présence d'une faille n'a été observé.

En examinant une carte topographique de la région du nord du Lac St-Jean, nous observons que le linéament est paral lẹ̀e à un réseau de ruisseaux (ou riviēre) secondaire orienté $320^{\circ}$, qui se rattache a un réseau primaire, marqué par les nombreuses rivières du nord du Lac St-Jean (riv. Mistassini, riv. Ouasiemsca, riv. Péribonca, etc.) orientées nord-sud. Sur la figure 5 nous avons indiqué le réseau hydrographique sommaire ainsi que 
l'emplacement des failles et axes de plis relevés dans la rêgion par Laurin et Sharma (1975).

Nous constatons que ces failles et axes de plis sont parallèles aux linéaments régionaux (nord-sud et $320^{\circ}$ ) et aux structures du complexe de Crevier $\left(320^{\circ}\right)$. Il semble donc que les failles et axes régionaux aient pu agir comme contrôle structural à la mise en place du complexe.

Le fort rubannement de 1 'unité 1 pourrait être le résultat du "débitage" de l'encaissant par des efforts de tension suite au relâchement d'efforts de compression. Lors de l'intrusion du massif, le magma a pu se mettre en place dans ces zones de roches débitées, agissant comme des zones de faiblesse facilement accessibles lors de la montée de l'intrusion. Par contre la nature massive de l'unité 2 ne peut être expliquée par le même processus que l'unité 1,Philpotts(1970) a proposé dans le cas des Montérégiennes, un mécanisme de mise en place au point de rencontre de failies conjuguées. Cette forme de contrôle structural pourrait s'appliquer dans le cas du complexe de Crevier. Il existe de tels réseaux dans la région du complexe (figure 5). Mais aucune évidence de failles perpendiculaire au linéament qui traverse le complexe, n'a été observé bien que les travaux de terrain n'étaient pas axés sur la recherche de telles structures.

La combinaison des deux types d'éléments structuraux décrits précédemment, pourraient expliquer la configuration peu commune du complexe de Crevier. Des travaux. sur la structure de l'encaissant seraient nécessaires pour confirmer ce modèle de contrôle structural. 


\section{FIGURE 5}

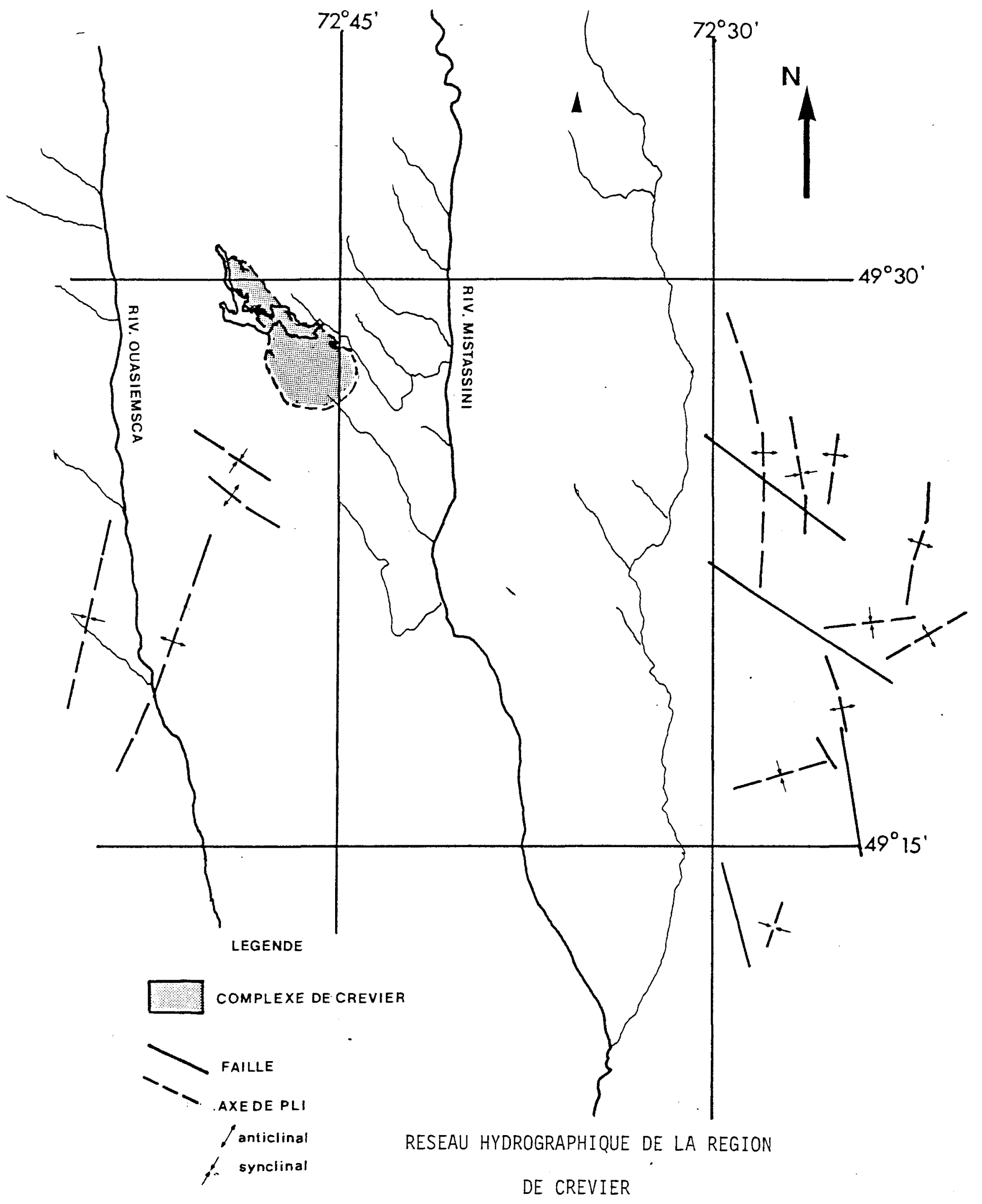




\subsection{Succession chronologique des événements intrusifs}

Le schéma de l'évolution chronologique de l'intrusion des différents types de roches du complexe; ainsi que des phénomènes de mêtasomatisme de la roche encaissante, qui accompagnent l'intrusion, ont pu être établis à partir des observations faites sur le terrain et dans les forages. Les événements, à partir du plus ancien, sont: (voir le tableau 2, résumant ces événements).

\section{1) Métasomatisme I de I'ençaisssant}

Le phénomène est observé sur une distance de $2 \mathrm{~km}$, il forme une auréole autour du complexe dans les roches de l'encaissant. La présence d'aégyrine dans ces roches est l'indice prédominant de métasomatisme. Les enclaves de gneiss oejllés métasomatisés observées dans la zone de contact, sont plus ou moins digérées par la monzosyénite à néphéline I (planche 10). La texture gneissique initiale est encore discernable, marquée par l'orientation des aggrégats d'aégyrine. Ces observations confirment le stade précoce, dans la succession chronologique des événements, du métasomatisme de l'encaissant.

\section{2) Métasomatisme II de l'encaissant}

La deuxième forme de métasomatisme, est marquée par la présence de veinules d'aégyrine de quelques militimètres de largeur et visibles sur une longueur de 10 a $50 \mathrm{~m}$. Ces veinules, recoupent les roches métasomatisées (1) ainsi que les roches non métasomatisées à la limite extrême de l'auréole. 
Nous avons observē des veinules dans des enclaves de gneiss oeillës métasomatisēs et partiellement digérés par la monzosyënite à néphëline I (planche 11).

3) Intrusion du massif et métasomatisme III de I'encaissant

Les premières phases intrusives du massif sont la mise en place de la monzosyénite à néphéline I et de la diorite à biotite. Nous supposons que ces deux phases sont contemporaines car nous avons observé, dans plusieurs bandes juxtaposées de l'unité 1 , des enclaves de diorite à biotite dans la monzosyénite à néphéline 1 (planche 12), et des enciaves de monzosyénites à néphéline 1 dans la diorite à biotite. L'unité 2 présente les mêmes textures, mais à une échelle plus réduite (observé dans les forages - seuiement).

La mise en place de la monzosyếnite à néphêline 1 a produit un troisième métasomatisme dans l'encaissant. Il s'agit de la digestion par cette dernière, des enclaves de gneiss oeillés à divers degrés dans la zone de contact. Les enclaves affectées par cette digestion ont nettement subi les deux autres formes de métasomatisme (planches 10 et 11) et elles sont recoupées par la monzosyënite à néphêline et biotite III et les dykes tardifs de monzosyênite à néphéline IV (planche 14).

4) Monzosyénite à néphéline pegmatitique II et minéralisation tantalifère

La monzosyênite à néphétine II est la deuxième phase intrusive du massif de par ses relations avec la monzosyénite à néphểine I, décrites dans le sous-chapitre précédent. Les dykes et poches de monzosyénite 
à néphêline pegmatitique II sont recoupés par la monzosyénite à néphêline et biotite (planche I5).

\section{5) Bréchifification}

La bréchification a été définie ainsi, en regard des srtuctures agmatiques mentionnées précédemment et occasionnée par la mise en place de la monzosyénite à néphéline et biotite III. Elle recoupe la monzosyénite à néphêline I et la monzosyénite à néphêline pegmatitique II; et elle est recoupée par les dykes tardifs de monzosyénite à néphéline IV (planche I6).

\section{6) Intrusion de dykes st_tardif요}

Cette phase represente l'intrusion de dykes de monzosyénite à néphéline IV et de dykes de diorite à biotite. Les premiers types de dykes sont prēsents dans toutes les unités du complexe (planche I7 unité I, planche 16 unité 2 et planche 10 unité 4$)$. Les dykes de diorite à biotite ont été observês que dans l'unitê I (planche I8).

\section{7) Intrusion de la carbonatite et minéralisation uranifère.}

La mise en place de la carbonatite a affecté tout les types de roche du complexe et de l'encaissant, par la digestion des roches dans lesquelles elle s'est mise en place (planche 19). Nous avons observé, de la carbonatite dans les épontes des dykes de monzosyénite à néphéline IV ainsi que dans les gneiss et les pegmatites de l'encaissant métasomatisé, sous forme de veinules et/ou de plages erratiques. Comme mentionné précédemment, la minéralisation uranifère (bétafite) a été observée dans la carbonatite seulement. 


\section{8) Mouvements de blocs}

Nous avons observé de nombreuses zones de cisaillement de quelques centimètres d'épaisseur dans les roches du complexe. Nous en avons observé un grand nombre dans les trous de forage, ainsi que sur les affleurements de 7 'unité 2 , recoupant toutes les lithlogies citées précédemment (planche 20). Il est possible que les textures de fluage: observées dans l'unité 1 et les mâcles de déformation dans la carbonatite soient associés à ces mouvements de blocs, mouvements qui sont peut-être reliés à des déplacements régionaux tardifs du au graben du Saguenay 
TABLEAU 2

\begin{tabular}{|c|c|c|c|}
\hline \multicolumn{2}{|c|}{ EVENEMENT } & LITHOLOGIES & CRITERES STRATIGRAPHIQUE. \\
\hline 1 & Métasomatisme 1 & Encaissant & $\begin{array}{l}\text { Enclaves de gneiss aégirinisés digèrées parSN } 1 \text { et } \\
\text { recoupées par SN } 2 \text {, SNb et SN } 4\end{array}$ \\
\hline 2 & Mètasomatisme 2 & Encaissant & $\begin{array}{l}\text { Veinules d'aég. recoupent les enclaves de gneiss mé- } \\
\text { tasomatisés }\end{array}$ \\
\hline 3 & $\begin{array}{l}\text { Intrusion du mas- } \\
\text { sif et métasoma- } \\
\text { tisme } 3\end{array}$ & SN 1 et $\mathrm{Db}$ & $\begin{array}{l}\text { Enclaves de SN } 1 \text { dans Db et de Db dans SN 1, digestion } \\
\text { de l'encaissant }\end{array}$ \\
\hline 4 & $\begin{array}{l}\text { Minéralisation } \\
\text { tantalifère }\end{array}$ & SN 2 pegmatitique & Dykes recoupant SN 1 et les enclaves metasomatisées \\
\hline 5 & Bréchification & SNb. & $\begin{array}{l}\text { Essaims de dykes recoupant SN } 1, \text { SN } 2 \text { et les encla- } \\
\text { ves métasomatisées }\end{array}$ \\
\hline 6 & $\begin{array}{l}\text { Intrusion de } \\
\text { dykes tardifs }\end{array}$ & SN 4 & Recoupent les lithologies anterieures, \\
\hline 7 & $\begin{array}{c}\text { Intrusion du } \\
\text { massif et mi- } \\
\text { neralisation U }\end{array}$ & C & $\begin{array}{l}\text { Assimilation des lithologies antérieures } \\
\text {. }\end{array}$ \\
\hline 8 & $\begin{array}{l}\text { Mouvements de } \\
\text { blocs }\end{array}$ & $\begin{array}{l}\text { Encaissant et } \\
\text { roches du com- } \\
\text { plexe }\end{array}$ & Mylonites recoupant les lithologies antérieures \\
\hline
\end{tabular}




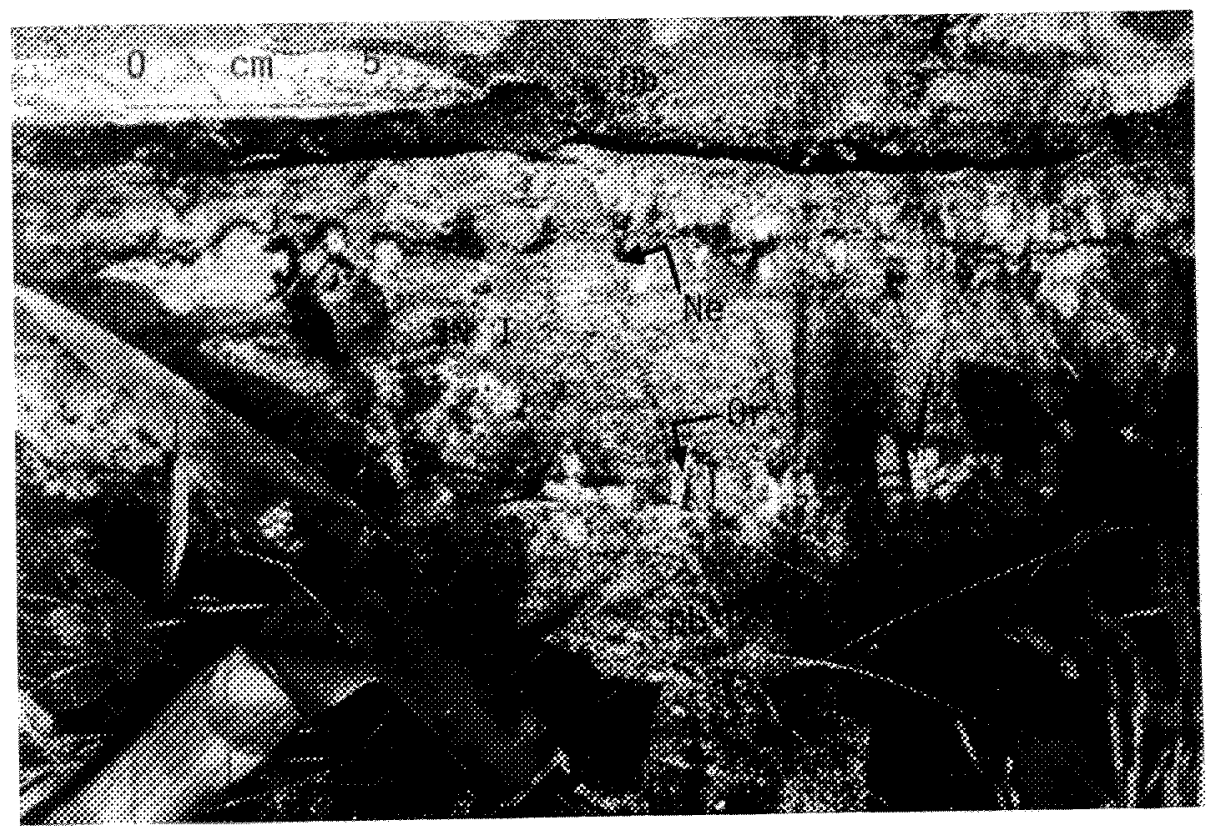

Planche 9: Photographie illustrant la disposition des phénocristaux d'orthose et de néphéline dans certaines bandes de monzosyênites à néphéline $I(S N)$ de l'unité 1 .

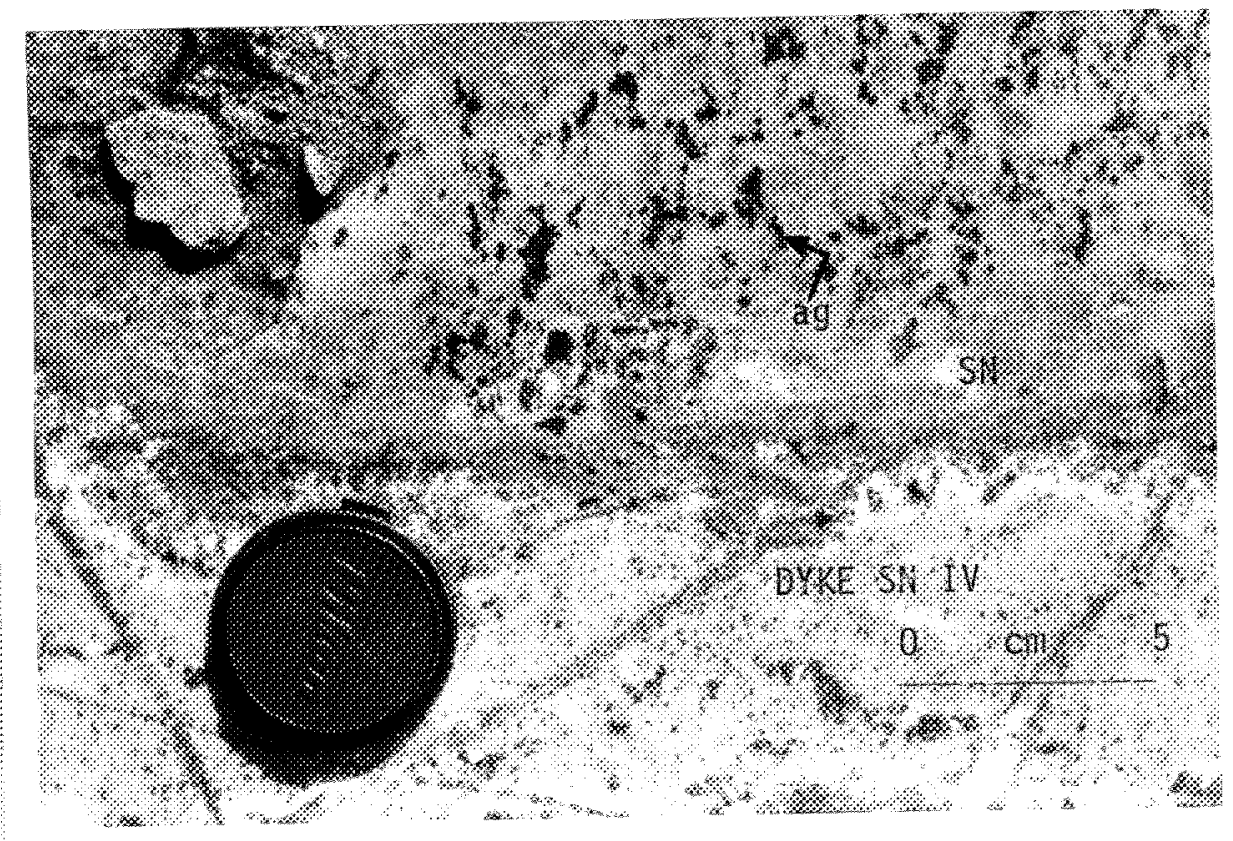

Planche 10: Photographie illustrant le gneiss oeillé digéré par la monzosyénite à néphéline I. Notons la concentration de nêphêline altérée (points blanchâtres) en bordure du dyke de monsosyénite à néphéline IV. 


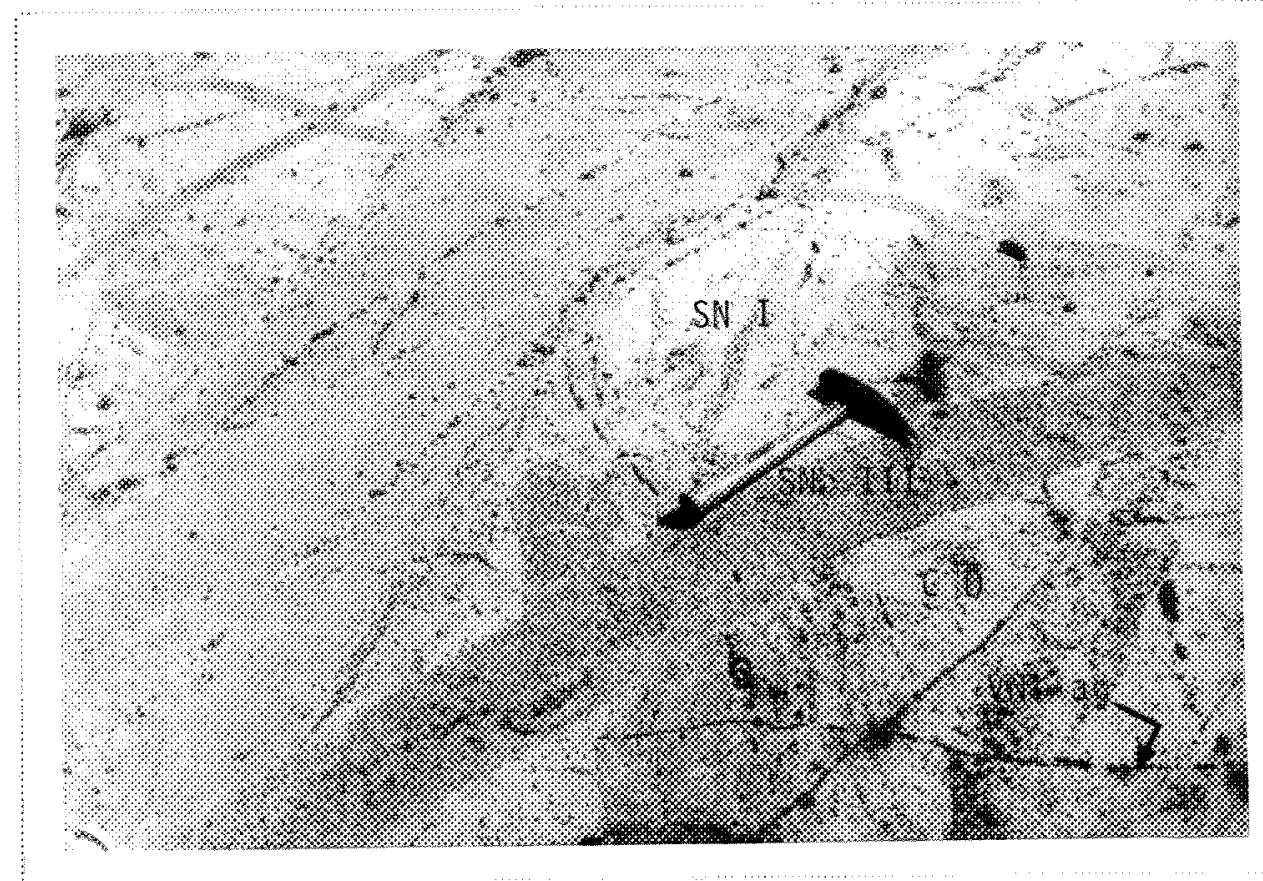

Planche 11: Photographie illustrant des veinules d'aégyrine (ag.) recoupant une enclave de gneiss oeillé (GNO) partiellement digéré par la monzosyénite à néphêline I. (unité 4)

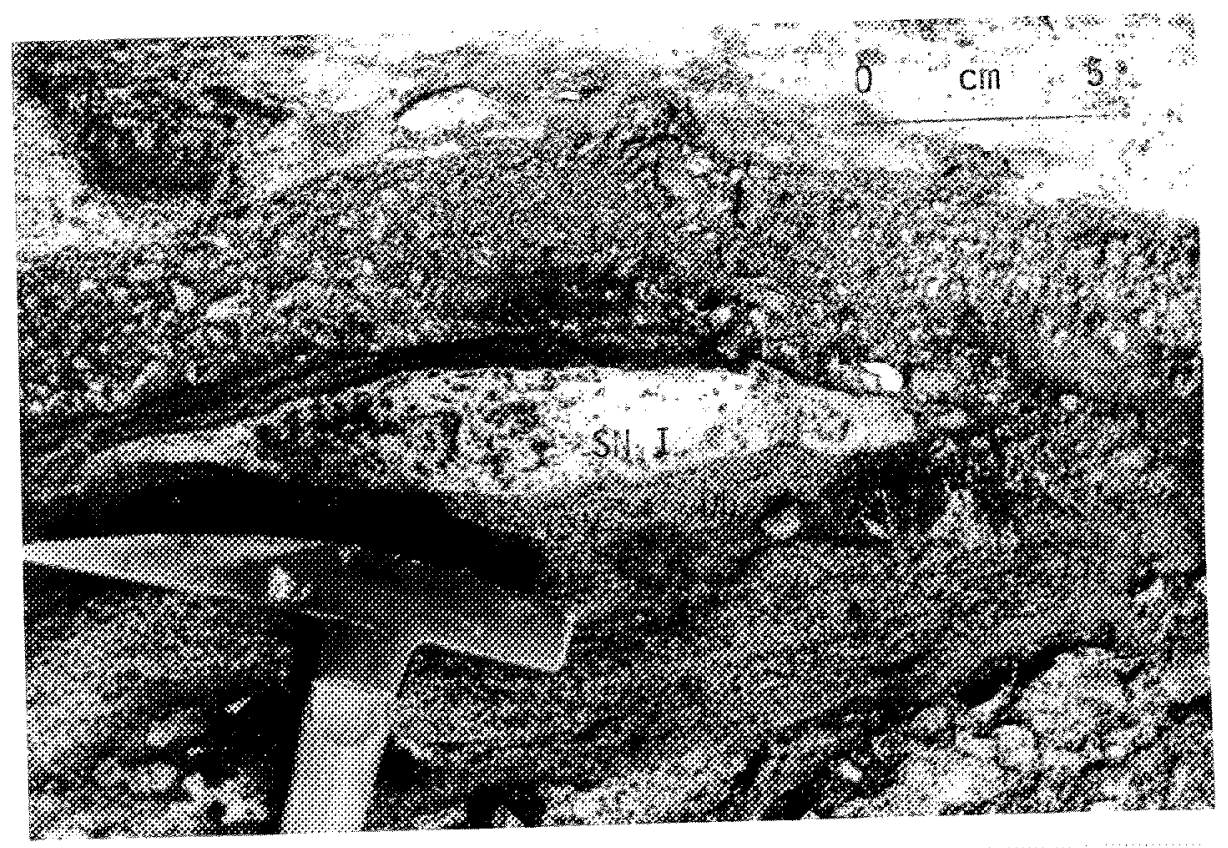

Planche 12: Photographie illustrant une enclave de monzosyénite à népheline I $(S N)$ dans la diorite à biotite $(D b)$, unité 1 


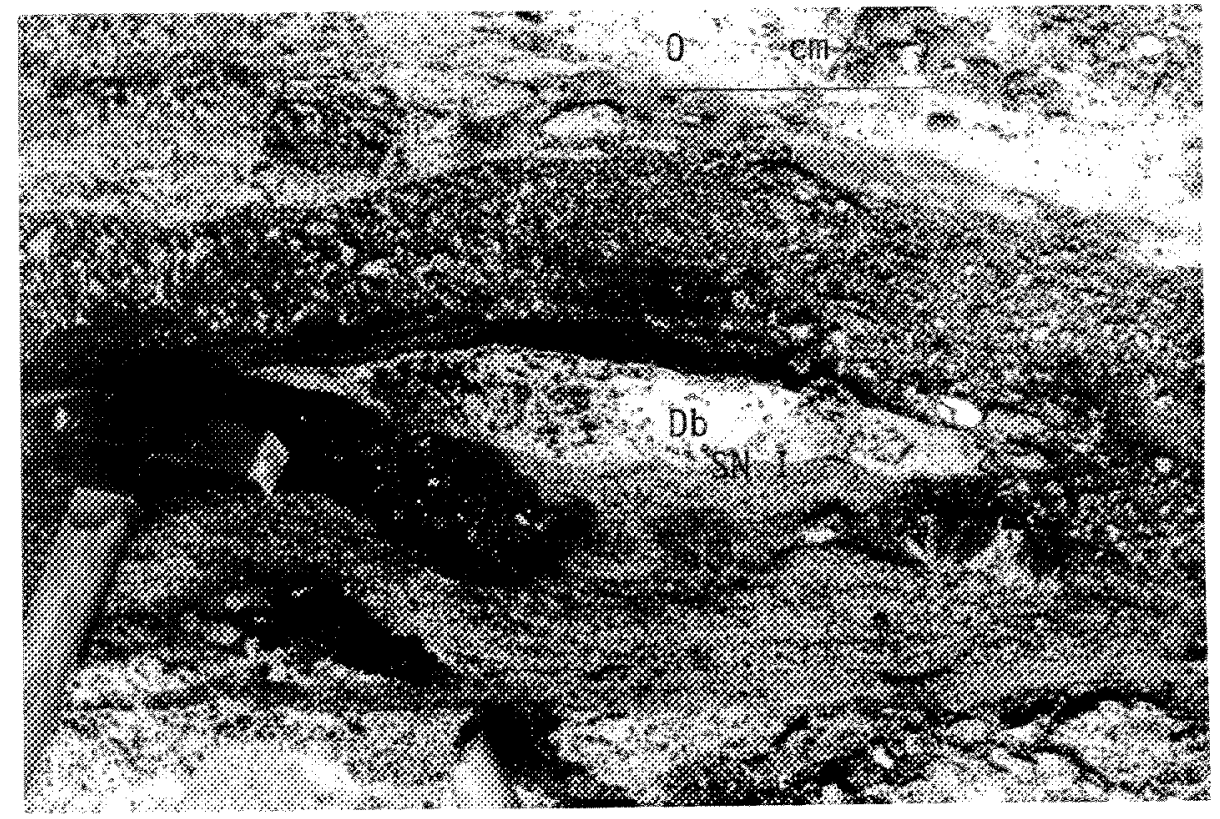

Planche 13: Photographie illustrant une enclave de diorite à biotite $(D b)$ dans la monzosyénite à néphéline I (SN), unte 1 .

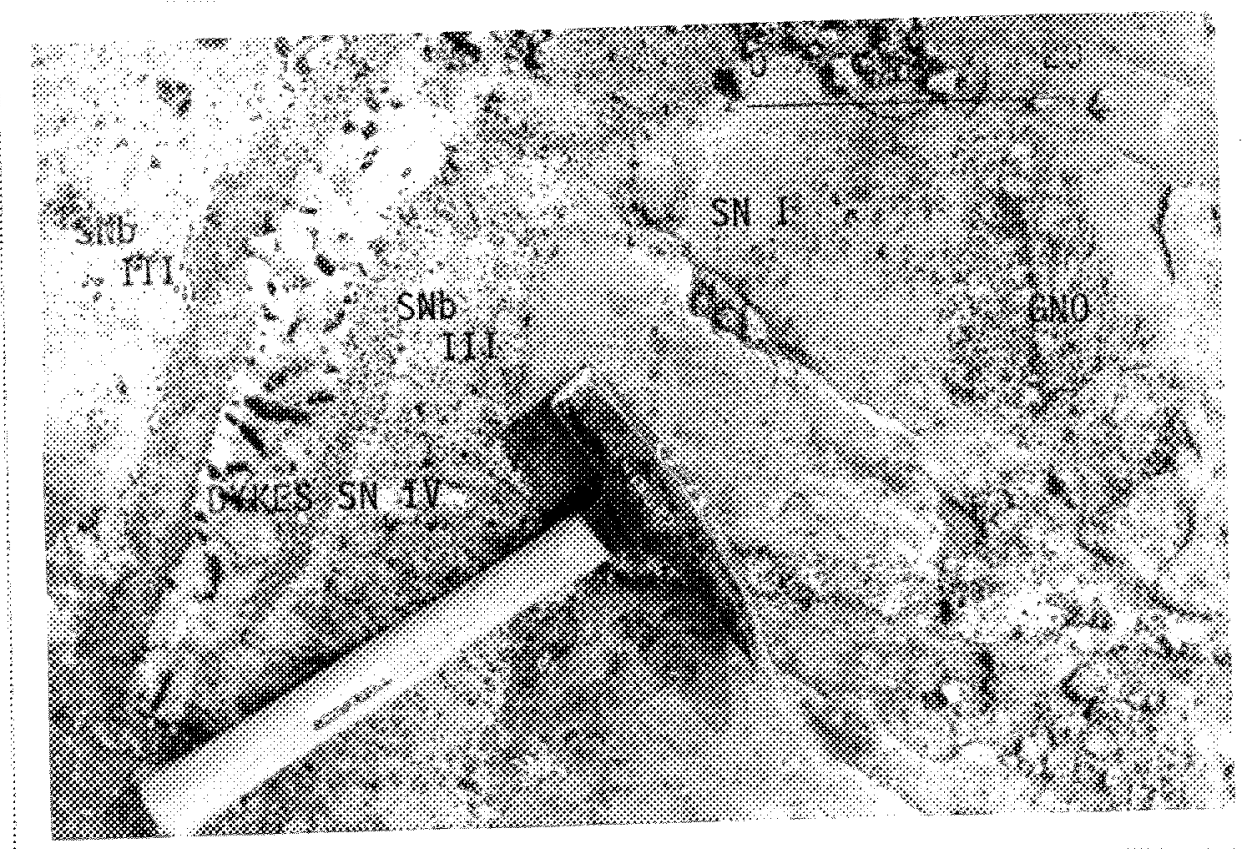

Planche 14: Photographie illustrant des dykes de monzosyénite à néphêline IV recoupant la monzosyénite à néphéline III (SNb) qui elle même recoupe la monzosyénite à néphéline I (SN), qui contient une enclave de gneiss oeillé (GNO), unité 4 . 


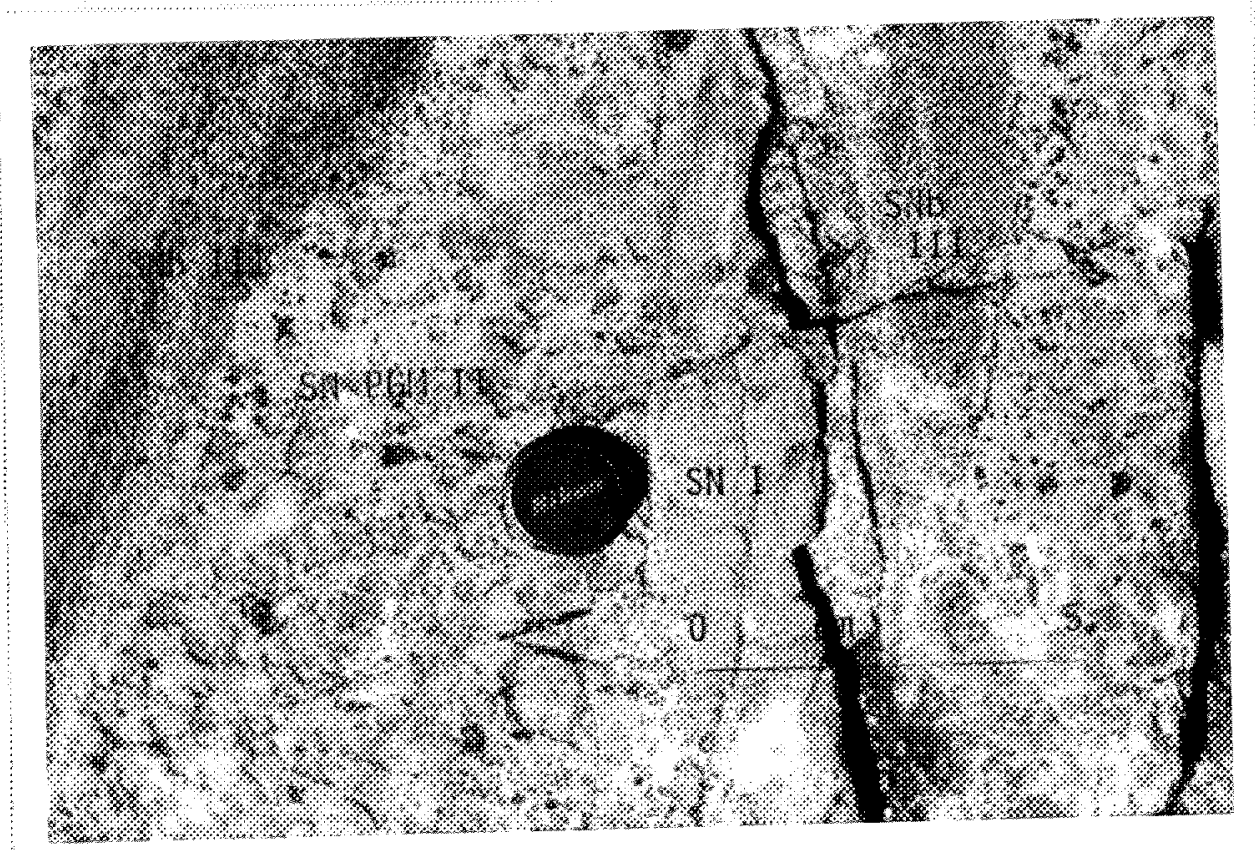

Planche 15: Photographie illustrant un dyke de monzosyênite à nêphêline pegmatitique II dans la monzosyénite à néphéline I $(S N)$, le tout recoupé par la monzosyénite à néphëline et biotite (SNb), unité 2 .

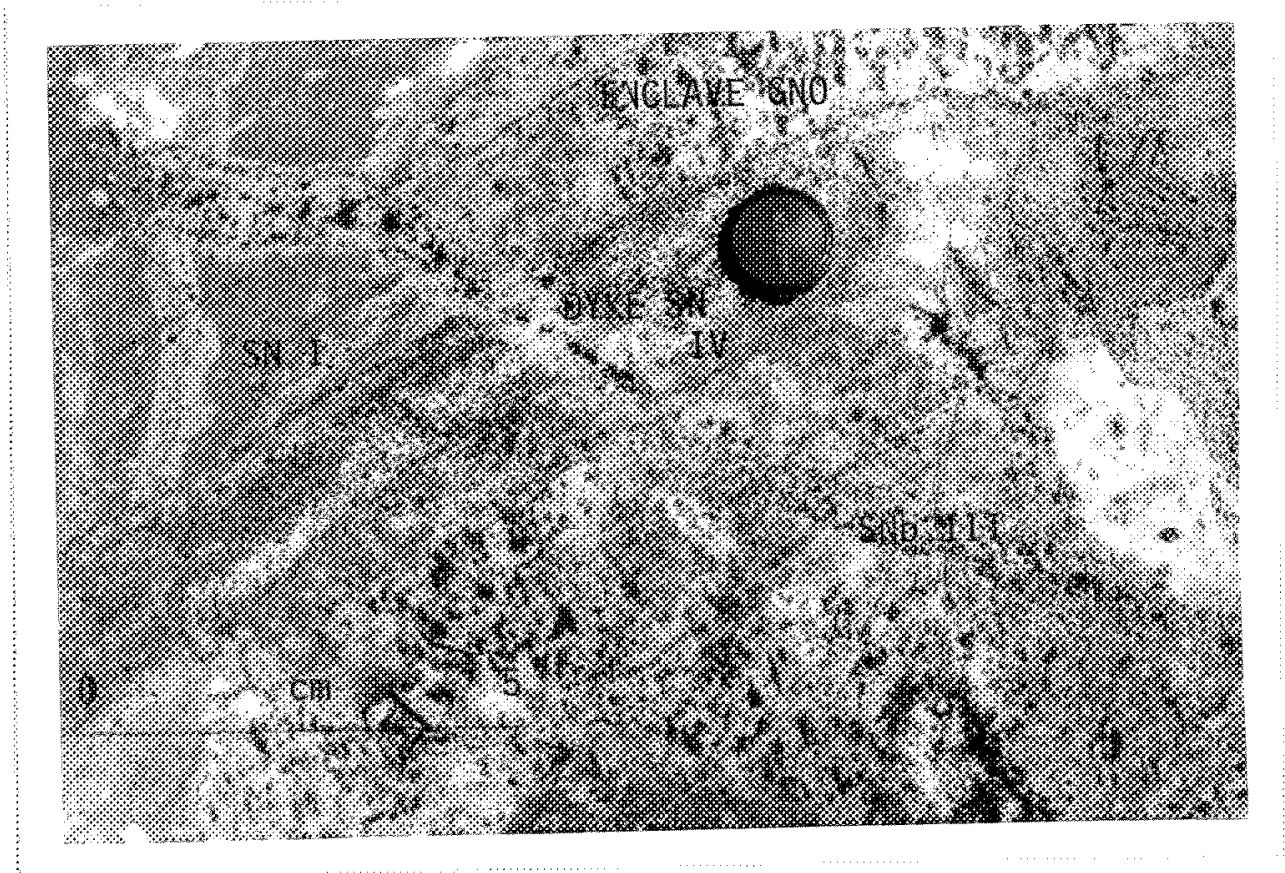

Planche 16: Photographie illustrant les relations stratigraphiques dans l'unité 2 . Nous observons une enclave de gneiss oeillê (GNO) dans la monzosyênite à néphêline I (SN) qui est recoupée par la monzosyénite à nêphẹline et biotite III (SNb) qui elle même est recoupée par la monzosyênite à manbetion TV 


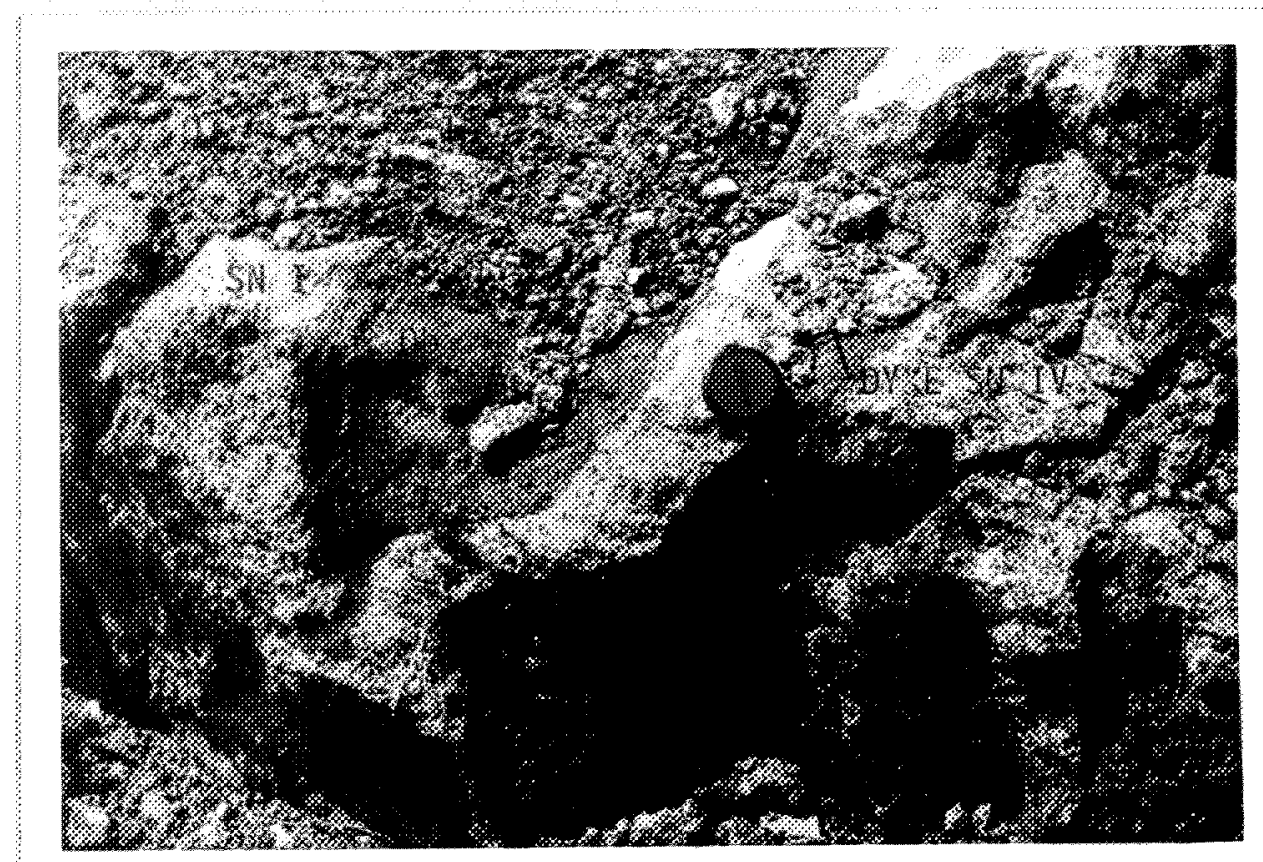

Planche 17: Photographie illustrant un dyke tardif de monzosyênite a néphêline IV recoupant la monzosyênite à néphêline I $(S N)$ et la diorite à biotite $(D b)$, unité 1 .

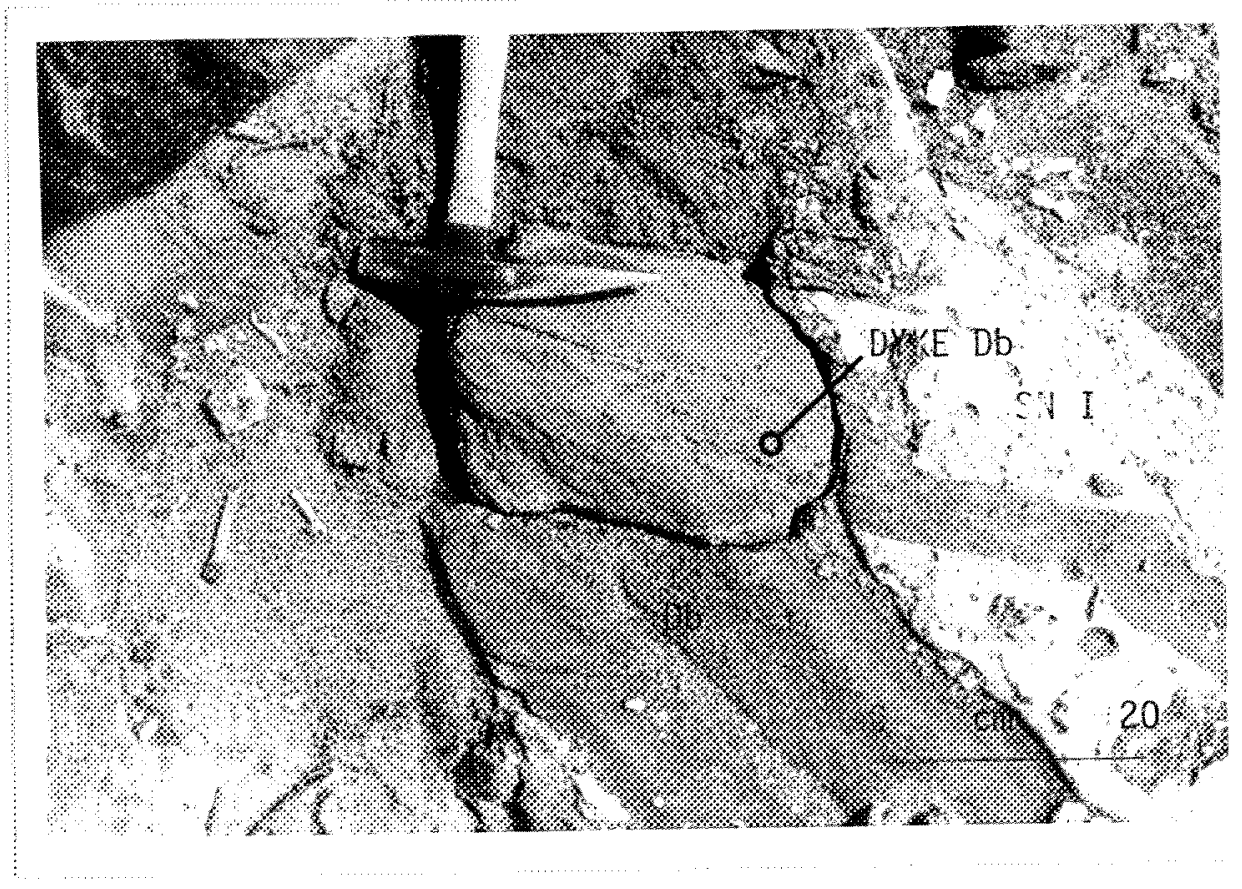

Planche 18: Photographie illustrant un dyke tardif de diorite à biotite (Db) recoupant la monzosyênite à néphéline I (SN) et la diorite à biotite, unité 1 . 


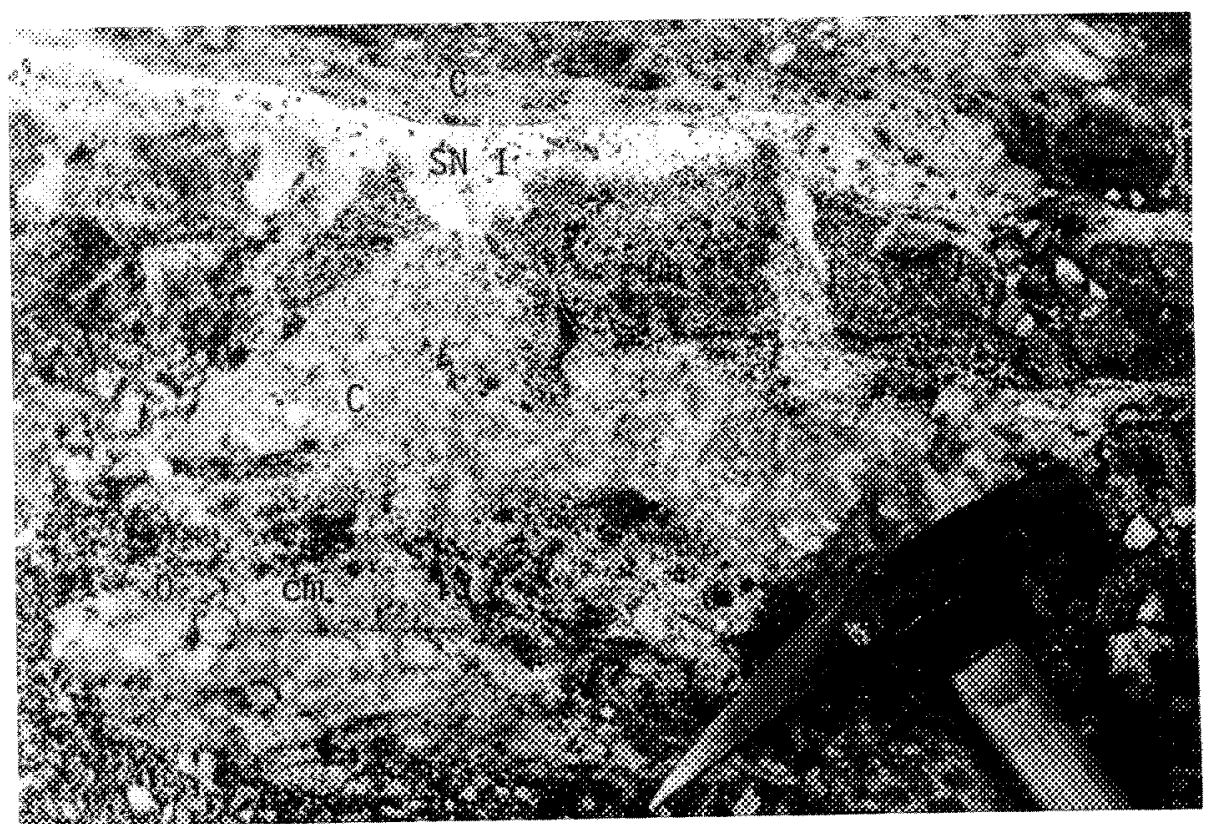

Planche 19: Photographie illustrant la digestion de la monzosyénite a néphêline I $(S N)$ et la diorite à biotite $(D b)$ par la carbonatite $(C)$, unité 1 .

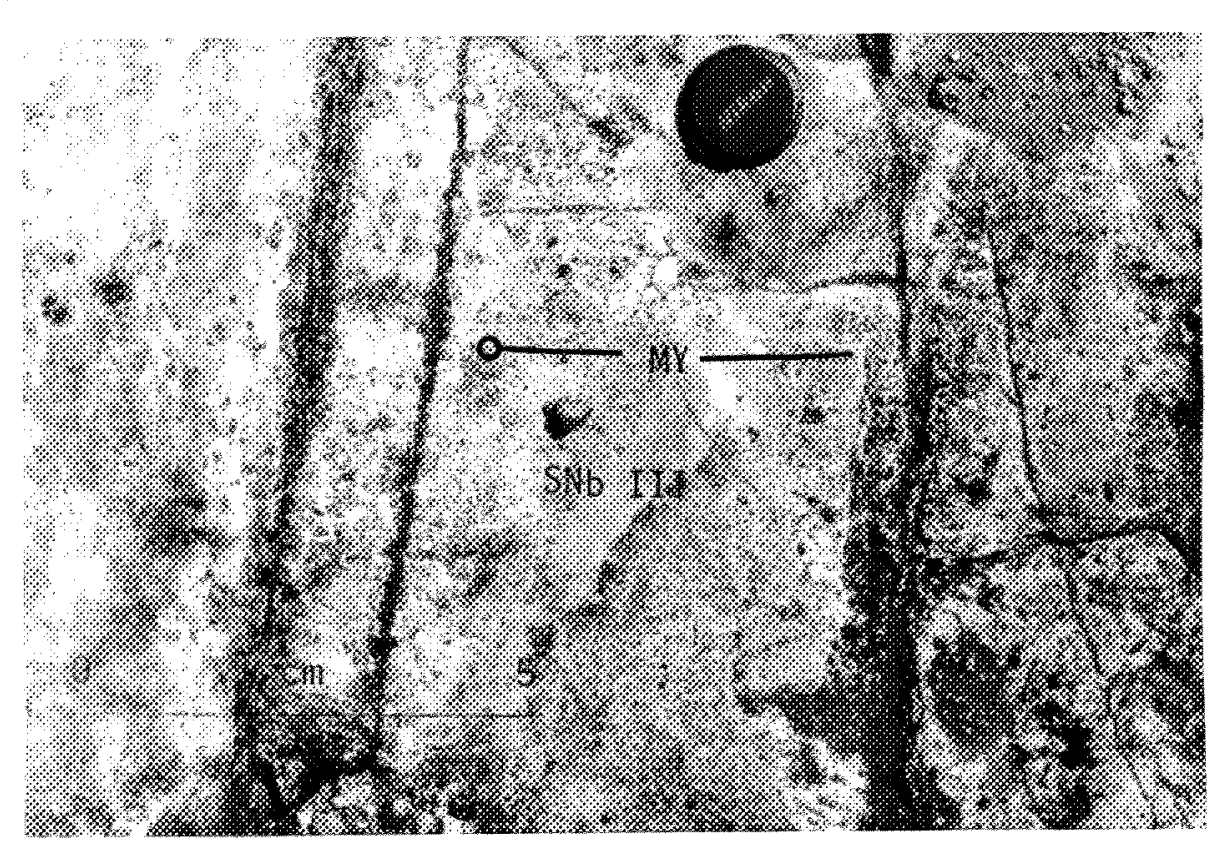

Planche 20: Photographie illustrant une zone de broyage (MY) recoupant la monzosyénite à néphéline et biotite (SNb), unité 2. 


\section{GEOLOGIE DE LA ROCHE ENCAISSANTE}

Ce chapitre a pour objectif de définir les caractéristiques géologiques de la roche encaissante fénitisée du complexe alcalin de Crevier.

Ces particularités comprennent la structure générale, les relations du contact pluton-encaissant, la pétrographie des principaux faciès et les effets du métasomatisme sur ces roches.

\subsection{Structures et relations du contact pluton-encaissant}

Le seui êlément structural qui a été relevé systêmatiquement, est la direction et le pendage du plan de foliation des roches de l'encaissant autour du complexe. Deux directions ont été relevées, l'une orientée $270^{\circ}$ et un pendage de $70^{\circ}-90^{\circ} \mathrm{N}$, dans les roches situées au nord et à $1^{\prime}$ 'ouest du complexe. La deuxième orientation est de $210^{\circ}, 70^{\circ}-90^{\circ} \mathrm{N}-0$, à l'est et au sud du complexe. Ces directions de foliation sont diffêrentes de l'orientation constante des éléments internes du complexe et n'ont pas de relations avec ce dernier.

Le contact entre les roches de l'encaissant et le complexe est progressif; nous l'observons sur une distance moyenne de $300 \mathrm{~m}$. Dans le massif, la proximité du contact est indiquée par la prêsence des enclaves de gneiss oeillés métasomatisés et complètement digêrês par la monzosyênite à néphêline I. Comme il a été dit précédemment, la foliation subsiste et son orientation est similaire à celle de l'encaissant $\left(270^{\circ}\right.$ dans les cas observés, 
cf. figure 4). Lorsque nous nous approchons de l'encaissant, nous observons une augmentation graduelle du nombre d'enclaves de gneiss et l'augmentation de leur grosseur.

A une distance approximative de $200 \mathrm{~m}$ de l'encaissant, les enclaves deviennent des blocs, la monzosyênite à néphéline forme des veines plus ou moins rectilignes entre les blocs de gneiss. Le tout forme une structure agmatique (Mehnert,1968); la grosseur des blocs et la largeur des veines de monzosyênite à néphéline I croissent et décroissent respectivement pour laisser place à l'encaissant métasomatisé. Parallèiement à l'augmentation du volume des blocs de gneiss, la digestion de ces derniers par la monzosyēnite à néphêline I diminue rapidement et n'est observable qu'au contact des veines avec les blocs, sur une distance de quelques $\mathrm{cm}$.

Les textures décrites et les relations structurales entre le complexe et l'encaissant, mettent en évidence le caractère intrusif du complexe alcalin a travers les roches du Grenville. Le complexe doit être relié à des événements post-orogéniques, possiblement à la formation du graben du Saguenay, site favorable à de telles genre d'intrusions et d'activité ignée alcaline (Kumarape11i et Saut, 1966; Bał̣ley, 1971)

\subsection{Pétrographie des principaux faciès}

L'étude pétrographique de l'encaissant du complexe se borne aux seuls faciès observēs dans un couloir de $4 \mathrm{~km}$ autour du massif. La nomenclature utilisée est celle de Laurin et Sharma (1975). 
Les faciès seront décrits de façon à pouvoir évaluer les rẻactions et les changements de l'assemblage minéralogique, que la roche a subi lors du métasomatisme. Les faciès identifiês sur le terrain sont les suivants:

- Les gneiss deillés (GNO)

- Les gneiss quartzo-feldspathique a biotite et hornblende (GNB)

- Les quartzites

- Les amphibolites

- Les pegmatites

\section{2-1 Les gneiss oeillés}

Les gneiss oeillés représentent le faciès majeur des roches qui affleurent autour du complexe. Leur morphologie varie en fonction de leur degré de recristallisation et de migmatisation. Les gneiss oeillés les moins touchés par la migmatisation, sont de couleur brune en surface àt térée et en surface frâche. Ils sont composés d'yeux de feldspath de 1 à $2 \mathrm{~cm}$ de diamètre, plus ou moins alignés dans le plan de foliation. Les yeux de feldspath baignent dans une matrice quartzo-feldspathique a grains fins, dans laquelle les minéraux mafiques sont interstitiels et montrent une forte linéation dans le plan de foliation. Les gneiss oejllés les plus touchés par la migmatisation montrent un aspect assez similaire à celui dēcrit précédemment, les principaux changements sont l'homogénéisation de la matrice quartzo-feldspathique avec les yeux de feldspaths, qui forment une "pâte" homogène à grains fins, de forme ovotide dont le grand axe est parallèle au plan de foliation. Les minêraux mafiques sont en bandes plus concentrëes, qui se moulent aux formes ovoides des yeux quartzo-feldspathiques. L'augmentation des contraintes tectoniques et de la fusion partielle produit l'étire- 
ment des formes oeillées, provoquant la formation de mêtatexites syncinêmatiques, c'est à dire la formation de bandes plus ou moins continues (boudinage) de quartz et de feldspaths à grains fins en alternance avec des bandes riches en minéraux mêlanocrates.

Les formes de gneiss oefiltés observés aux abords du complexe, sont surtout celles qui ont gardé leurstextures originales. La roche est composée de feldspaths alcalins, de plagioclases, de quartz, de biotite, de hornblende verte, d'augite et de zircon.

Les yeux de feldspaths sont composés d'orthose perthitique de type film, leur taille varie de 0.5 a $2 \mathrm{~cm}$. I Is contiennent un grand nombre d'inclusions de plagioclases damouritirisés en leur centre ainsi que des inclusions de microcline. Les yeux baignent dans une matrice granulaire à grains fins, composée de quartz, d'orthose légèrement séricitisée, d'orthose graphique, de microcline fraiche et de plagioclases (An 30) fortement damoutirisés.

Les minéraux mafiques sont composés d'aggrégats de biotite et de hornblende verte, dont la taille est de $0.2 \mathrm{a} 1 \mathrm{~mm}$. La biotite est tabulaire et contient de nombreuses inclusions de plagioclases, d'orthose et de microcline, certains grains sont partiellement chloritisés. La hornblende verte est interstitielle entre les aggrégats de biotite; elle est tabulaire, fortement fracturée, les bordures de grains sont hachurées et très irrégulières. L'augite est disséminée dans les aggrégats de biotite et de hornblende verte, elle est hypidiomorphe, de forme plus ou moins sphérique. Les minēraux mafiques sont orientés dans la roche, ils forment des bandes continues dans la matrice et ils ceinturent les yeux de feldspaths. 
Les accessoires sont composēs de sphēne et de zircon, ils sont interstitiels dans la matrice.

Les gneiss oeillés qui ont subi le métasomatisme I conservent les mêmes textures décrites précédemment, les changements sont d'ordre minéralogique. Au niveau des yeux de feldspaths nous observons l'augmentation progressive de la phase exsolvée, passant du type film au type veine. Dans les roches observées aux abords du complexe, la phase exsolvêe sort du grain et elle forme des grains d'albite ceinturant les yeux d'orthose. Au niveau de la matrice des roches faiblement métasomatisées, les plagioclases montrent des mâcles de glissement, ainsi que l'apparition de lamelles d'exsolution d'orthose. Les grains d'orthose et de microcline montrent une forte fracturation. Les aggrégats mafiques sont caractérisés par l'apparition d'aégyrine et d'arfvedsonite et, rapidement, l'aégyrine devient la phase mafique majeure de la roche. La formation d'aégyrine se fait progressivement aux dépends du quartz de la matrice; elle ceinture

les grains de quartz et pënètre dans ces derniers pour les remplacer complètement. Les feldspaths alcalins et les plagioclases sont aussi remplacês, illustré par la présence de nombreuses inclusions de ces derniers dans les grains d'aégyrine. Les roches fortement fénitisées sont caractērisées par le remplacement quasi total des minéraux qui composaient la matrice originale de la roche, ainsi que le début de remplacement des yeux de feldspaths. La hornblende. verte et l'augite sont les seuis minéraux qui subsistent; nous n'observons aucun changement minéralogique dans leur cas. Le rôle de la biotite n'est pas défini avec précision, bien qu'aucune texture de remplacement de la biotite par l'aégyrine n'ait été observé, la biotite est la première phase minérale, avec le quartz, qui ne compose plus l'assemblage minéralogique original. 
L'arfvedsonite n'est observée que dans les roches qui montrent un faible métasomatisme; elle remplace l'aégyrine, formant des couronnes partielles autour de cette dernière.

Dans les roches métasomatisées nous observons de la calcite en plus ou moins grande quantité, elle forme des plages xénomorphes de 1 a $5 \mathrm{~mm}$; de l'apatite interstitielle ainsi qu'un fort pourcentage de zircon et de sphène (1\%) par rapport au contenu de la roche originale (traces).

\section{2-2 Les gneiss quartzo-feldspathiques à biotite et hornblende}

Les gneiss quartzo-feidspathiques à biotite et hornblende, sont fréquemment observés dans la partie sud-est de l'encaissant du complexe. Les gneiss sont homogènes dans leur composition minéralogique mais leur degré de fusion partielle et de recristallisation leur confère une forte hétérogénéité quant à leur aspect mégascopique. La migmatisation produit une ségrégation partielle et/ou totale des constituants leucocrates et mélanocrates de la roche. La ségrégation se traduit par l'alternance de bandes riches en minéraux mafiques avec des bandes riches en minéraux quartzo-feldspathiques. Le passage d'une bande à l'autre est graduel et en général, les bandes leucocrates sont plus épaisses (1 à $2 \mathrm{~cm}$ ) que les bandes mélanocrates $(0.1 \mathrm{a} 1 \mathrm{~cm})$. Les roches qui ne comportent pas de ségrégation ont un aspect massif, bien que la foliation est facilement discernable par l'alignement des minéraux mafiques dans le plan de foliation. Les gneiss quartzo-feldspathiques a hornblende et biotite sont composês de quartz, de feldspaths alcalins, de plagioclases, de biotite, de hornblende verte et de zircon. 
Le quartz est sous forme d'aggrëgats de grains polygonaux, de taille moyenne contenant des inclusions de plagioclases, de feldspaths alcalins et de biotite. Les feldspaths alcalins sont hypidiomorphes et leur taille est de $2 \mathrm{~mm}$. Ils sont composés de microcline et d'orthose perthitique de type film. Ils contiennent de nombreuses inclusions de quartz. L'orthose forme occasionnellement des textures graphiques avec le quartz.

Les plagioclases (An 28) sont hypidiomorphes; ils sont de forme tabulaire et de même taille que les feldspaths alcalins.

Les minéraux mafiques sont composês principalement de biotite tabulaire; les grains sont allongés et leur taille est de 1 a $2 \mathrm{~mm}$. La hornblende est xénomorphe; ses bordures de grains sont irrégulières et leur taille est de 1 a $2 \mathrm{~mm}$. Elle possède une texture poéçilitique contenant un grand nombre d'inclusions de quartz. Les mafiques sont disposés en bandes plus ou moins continues de 1 à $5 \mathrm{~mm}$ d'épaisseur. Les bandes contiennent des aggrégats de biotite-hornblende ou de biotite seu1e.

Les roches affectées par le métasomatisme I présentent des textures similaires au gneiss oeillê, mais les rêactions sont confinées aux horizons mafiques. L'aégyrine remplace le quartz et probablement la biotite: nous observons l'augmentation de la phase exolvêe dans les feldspaths alcalins, ainsi que la fracturation de leur grains, et les plagioclases sont antiperthitiques. L'arfvedsonite remplace l'aégyrine dans les roches peu métasomatisées et enfin nous notons la présence de plages de calcite erratiques, de zircon, de sphène et d'apatite dans les roches 
fortement métasomatisées. La hornblende verte garde ses formes et sa texture originale, la texture gneissique de la roche est aussi préservée.

\section{2-3 Les quartzites}

Les quartzites n'ont été vues qu'à un seul endroit sur le terrain. Il s'agit d'une petite bande pincée dans les gneiss quartzofeldspathiques à biotite et hornblende, située à proximi té du contact du massif alcalin. Les reáactions métasomatiques sont très fortes et ont produit l'aégyrinisation quasi complète de la quartzite. Il ne subsistait que quelques lambeaux de roche non al térée, composée de bandes riches en feldspaths $(10-15 \%)$ et de bandes de quartz.

\section{2-4 Les amphibolites}

Les amphibolites sont très peu observêes dans l'encaissant immédiat du complexe. Elles forment des enclaves en forme de croissant et leur taille est de 0.2 par $0.5 \mathrm{~m}$.

La roche est de couleur noire, à grains fins. Elle est composé de hornblende verte, de clinopyroxene, de plagioclases et de quartz. La hornblende verte est hypidiomorphe, les bordures de grains sont plus ou moins hachurées. Le pyroxène est une augite hypidiomorphe de forme sphérique, interstitielle dans la hornblende verte. Les plagioclases sont xénomorphes, ils sont à grains fins et fortement damouritirisés (An inconnu) Nous notons la présence de quartz interstitiel. 
Les échantillons métasomatisés ne montrent pratiquement pas de changements minéralogiques et texturaux. La hornblende verte, l'augite et les plagioclases restent intacts. Nous avons observé de l'aégyrine en traces, associee au quartz interstitiel. Les carbonates sont présents sous forme de plages xénomorphes, interstitielles dans la roche. Les accessoires sont composés de zircon, d'apatite et d'opaque. L'amphibolite, bien que recueillie dans une zone de métasomatisme relativement êlevé, à résisté au métasomatisme et ne montre que des changements minéralogiques mineurs.

\section{2-5 Les pegmatites}

Nous portons une attention particulière à la description de ce type de roches, car les pegmatites sont les seules roches de l'encaissant qui représentent une homogénéité texturale et minéralogique constante, et de ce fait, ont été choisies pour faire l'évaluation pétrographique, qualitative et quantitative du métasomatisme. Les pegmatites serviront aussi de critère stratigraphique pour situer 7 'intrusion du massif par rapport à l'orogënie grenvillienne. Selon nos observations sur le terrain,elles semblent être les seules roches de l'encaissant qui sont peu dêformées donc largement post-orogénique.

Les pegmatites forment des dykes rectilignes de quelques centimètres à $0.5 \mathrm{~m}$ de largeur, qui recoupent toutes les lithologies décrites antêrieurement. Elles sont de couleur blanche en surface ai têrée et rougeâtre en surface fraiche. Leur morphologie est relativement simple; elles sont composés d'aggrégats de quartz au centre du dyke et de 
gros cristaux de feldspaths, en bordure (planche 21). La roche est composêe de feldspaths alcalins, de plagioclases, de biotite, de zircon et d'opaques. La muche est très grenue, la taille des grains varie entre $4 \mathrm{~mm}$ et $2 \mathrm{~cm}$.

Les feldspaths alcalins sont composés de gros cristaux de microcline et d'orthose perthitique de type veine. Ils sont frais et légèrement fracturés; ils contiennent des inclusions de quartz. Les plagioclases (An 31) sont tabulaires et leur taille est de 0.5 a $2 \mathrm{~cm}$. Ils sont frais et quelques grains sont zonés. Le quartz est sous forme de plages interstitielles entre les feldspaths, qui contiennent des grains xénomorphes de 1 à $5 \mathrm{~mm}$. Quelques uns ont une extinction roulante. En gënéral, tous les minēraux sont interlobés. Au contact des feldspaths alcalins et des plagioclases, nous observons localement, des petits grains de même composition soit d'orthose, de microcline, de plagioclases, de quartz et d'orthose graphique provenant de la recristallisation partielle des gros cristaux; la roche possède par le fait même une texture en mortier développée localement donc peu dêformée. La biotite est de couleur brune-orangée; ellè est à grains fins et de forme asciculaire. Elle forme des aggrégats dont les grains sont disposés en rosette dans les plagioclases (texture symplectique) et dans les minéraux à grains fins. La planche 22 illustre la texture et l'assemblage minéralogique général de la roche. Les accessoires sont le zircon et les opaques, ils sont interstitiels dans la roche. 
Les premiers indices de métasomatisme sont l'apparition de l'aégyrine et de l'arfvedsonite (2-5\%) dans les aggrégats de quartz (planche 23). L'arfvedsonite forme des couronnes partielles autour des grains d'aégyrine, remplacant cette dernière. L'aégyrine contient de nombreuses inclusions de feldspaths alcalins. Les grains d'orthose et de microcline non remplacés sont fortement fracturés. Les gros cristaux de feldspaths alcalins ne subissent pas de changements majeurs; nous observons une augmentation sensible du volume de la phase plagioclasique des perthites. Au niveau des plagioclases, nous notons l'apparition d'une phase feldspathique (orthose) à l'intérieur des grains, ainsi que là présence de mâcles de glissement de certains individus. Notons que la biotite ne compose plus l'assemblage minéralogique original. Le métasomatisme n'a pas altérê la texture englobée des différents minéraux ainsi que l'aspect granuleux de la roche. Les minéraux accessoires sont composés de zircon, de sphène et d'apatite. Ils sont interstitiels dans la roche. La planche 24 illustre les effets du métasomatisme sur la minéralogie et la texture de la roche.

A un niveau de métasomatisme plus élevé, les changements de la minéralogie sont beaucoup plus évidents. L'aégyrine est devenue la phase mafique majeure de 1 a roche (planche 25), composant de 10 à $20 \%$ de cette dernière. Elle remplace tout le quartz et les minéraux à grains fins interstitiels aux gros cristaux de feldspaths et elle ne contient que des inclusions de microcline. L'arfvedsonite, le quartz, la biotite et l'orthose ne composent plus l'assemblage minéralogique original. Le volume de la phase exsolvêe des gros cristaux de feldspaths alcalins a consi- 
dérablement augmenté (40 - 50\% d'albite). Les mâcles polysynthétiques des plagioclases exsolvés sont nettement visibles; ils migrent à l'extêrieur du grain et ils forment des couronnes autour du gros cristal. Ce dernier contient un grand nombre de grainsde zircon, d'apatite et de calcite. La calcite forme des plages alongées dans le grain, disposées le long du plan de clivage du feldspath. Nous notons l'apparition d'une nouvelle phase minëralogique, composée d'aénigmatite en petits grains xénomorphes dont la taille est de 0.1 a $0.5 \mathrm{~mm}$. Ils sont interstitiels dans l'aégyrine ainsi que la calcite, le zircon et l'apatite. Bien que des changements soient importants à ce stade de métasomatisme (planche 26), la texture originale de la roche est conservée. Ce fait est bien illustrê par la comparaison mégascopique d'une pegmatite non-métasomatisée ( $p$ lanche 21 ) et une pegmatite fortement métasomatisée (planche 25 ). L'aspect de la roche est le même; I'aégyrine remplace les plages de quartz et de feldspaths et n'altère que très peu les gros cristaux̃ de feldspaths. La présence de veinules d'aégyrine dans les pegmatites est un indice de la possibilité que la roche a subi une augmentation de volume durant le métasomatisme.

Les effets du métasomatisme diffèrent selon le type de roche qu'il affecte. Les gneiss oeillés et les gneiss quartzo-feldspathiques a biotite et hornblende sont altérés surtout au niveau des horizons mafiques, qui composent ce type de roche. L'amphibolite n'est à peu près pas altérée et les pegmatites sont attaquées au niveau des aggrégats de quartz. La composition minéralogique de la roche parentale a un effet prédominant sur le produit final de l'altération métasomatisme. La structure interne de la roche, ainsi que sa granulométrie semble avoir aussi une grande influence sur les formes de métasomatisme. 
La création des assemblages minéralogiques observés dans les roches fénitisées, nécessitent des apports de matière assez considérables. A l'aide des études géochimiques qualitatives et quantitatives, qui feront l'objet d'un chapitre subséquent, et les observations pétrographiques que nous avons effectué; nous serons en mesure de proposer un modèle i17ustrant l'ensemble des réactions observées dans les différents types de roche. 


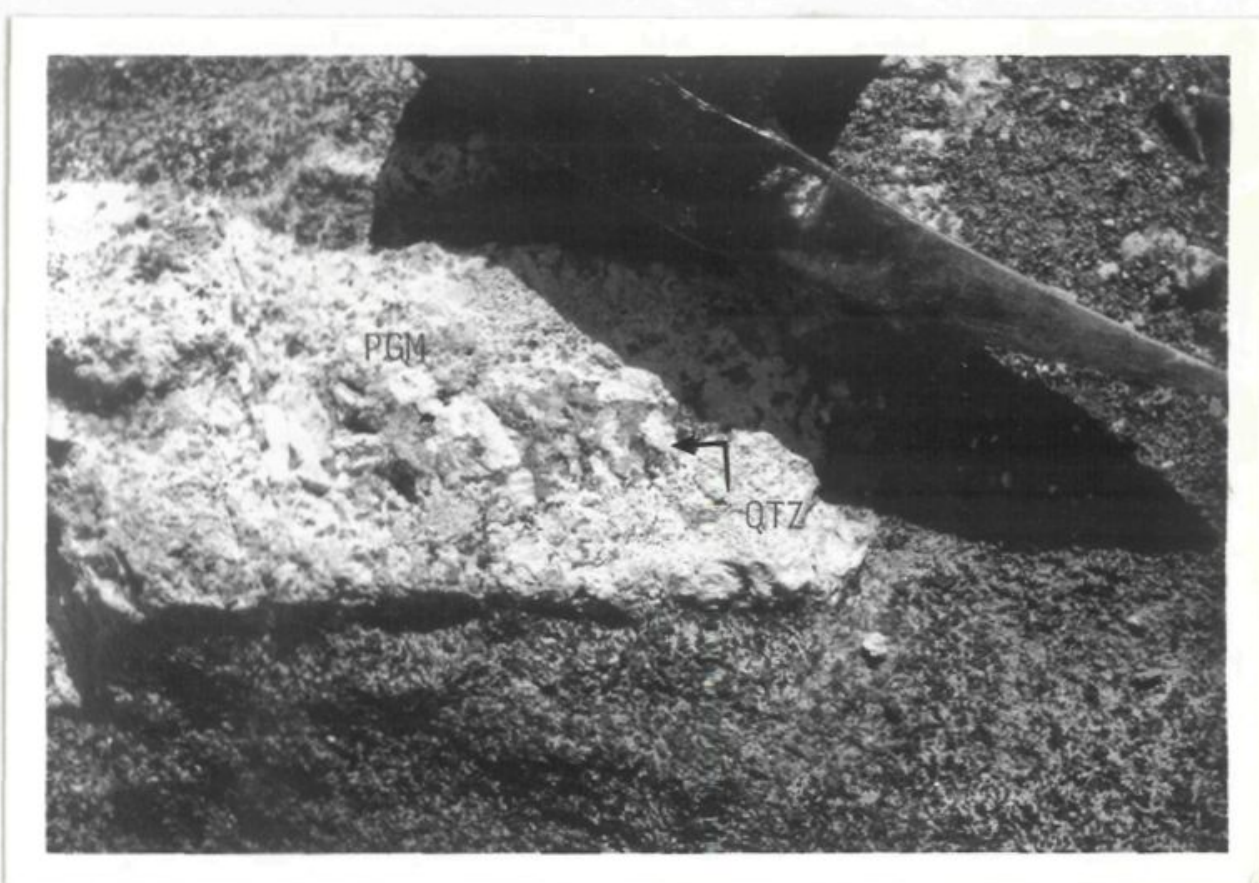

Planche 21: Photographie illustrant 7a texture générale des dykes de pegmatite (PGM) non-métasomatisées. Nous observons des aggrégats de quartz au centre du dyke et les feldspaths alcalins en bordure.

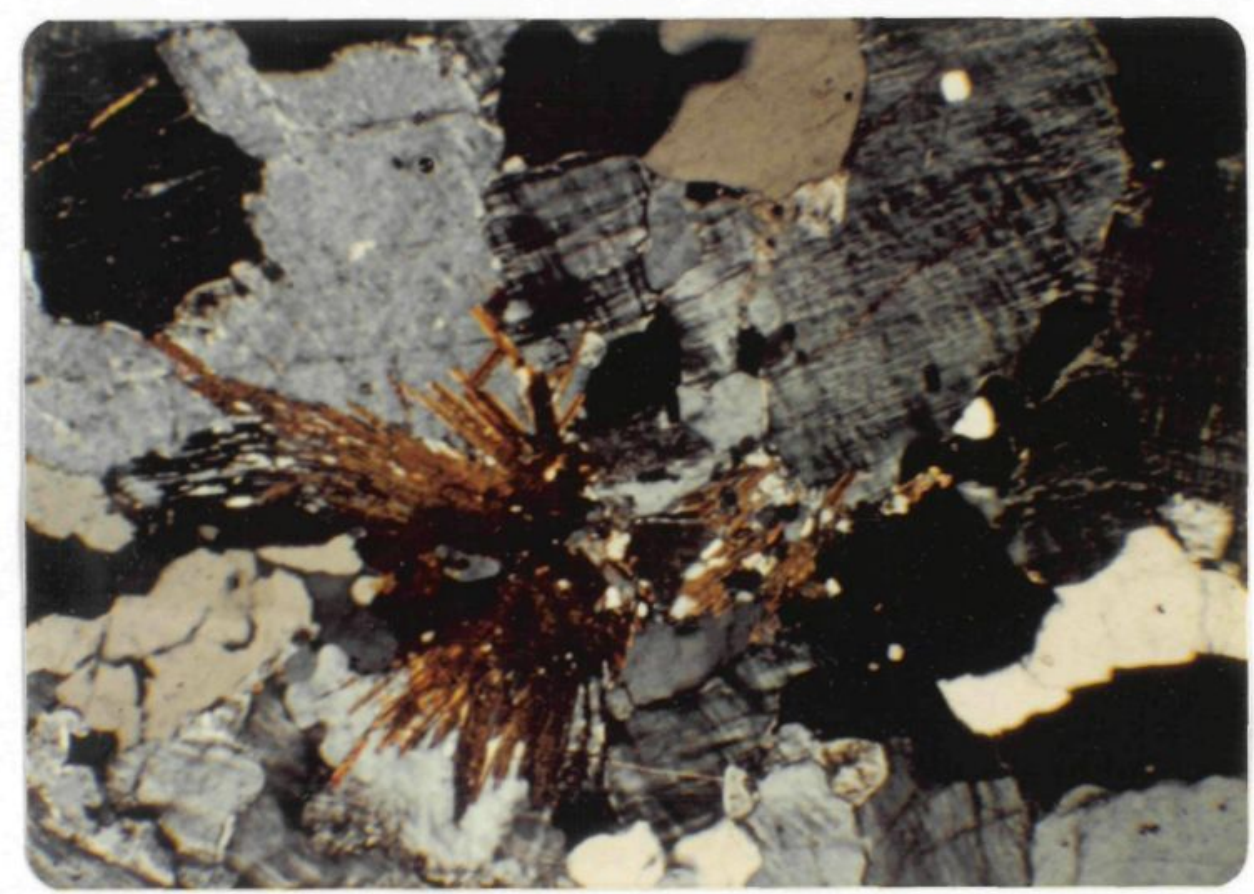

Planche 22: Microphotographie illustrant la minéralogie et la texture des pegmatites non-métasomatisées. Nous observons les cristaux de microcline perthitique, des plages de quartz et des aggrégats de biotite. $\varnothing=6.5 \mathrm{~mm}$ 


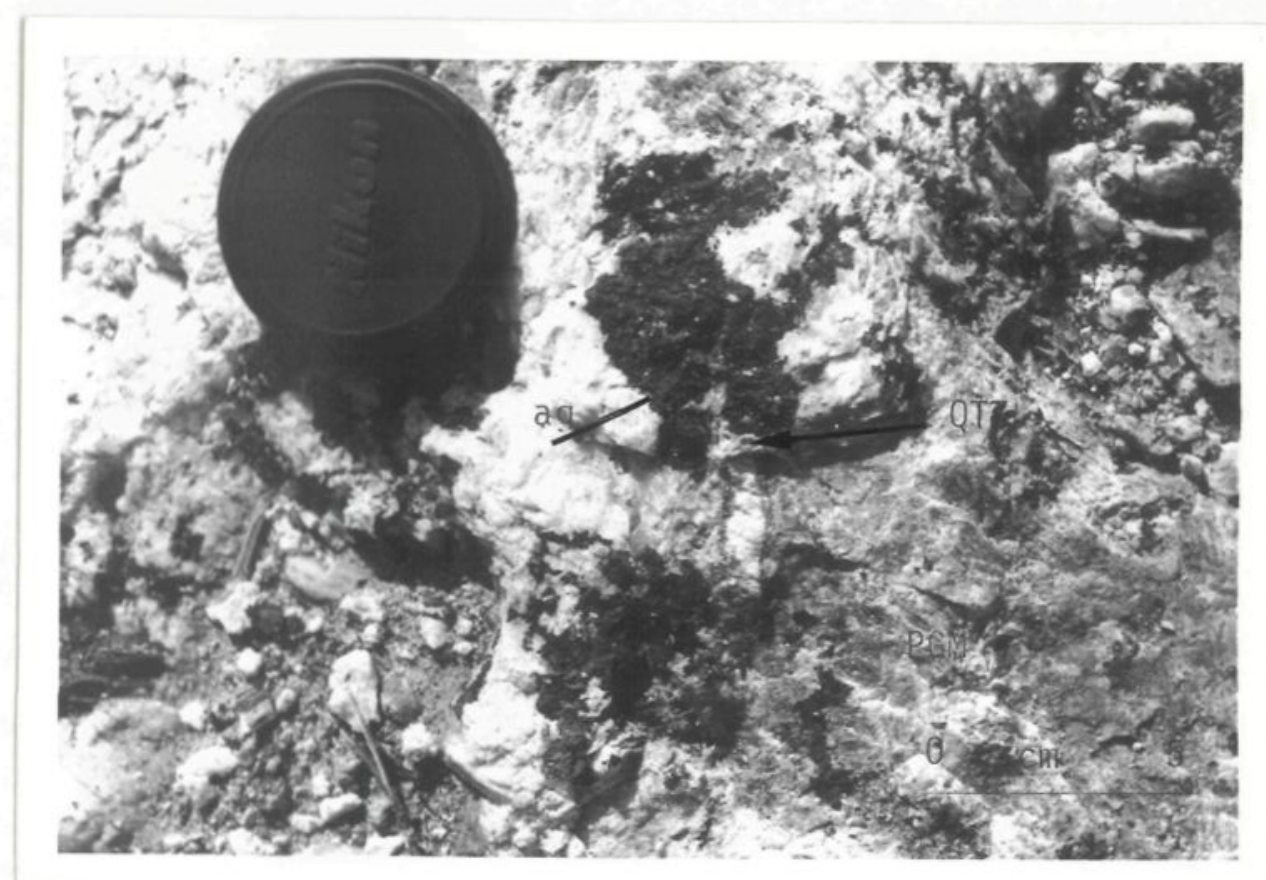

Planche 23: Photographie illustrant 1'aspect des pegmatites faiblement métasomatisées. Nous observons l'apparition des grains d'aégyrine (ag) dans les aggrégats de quartz

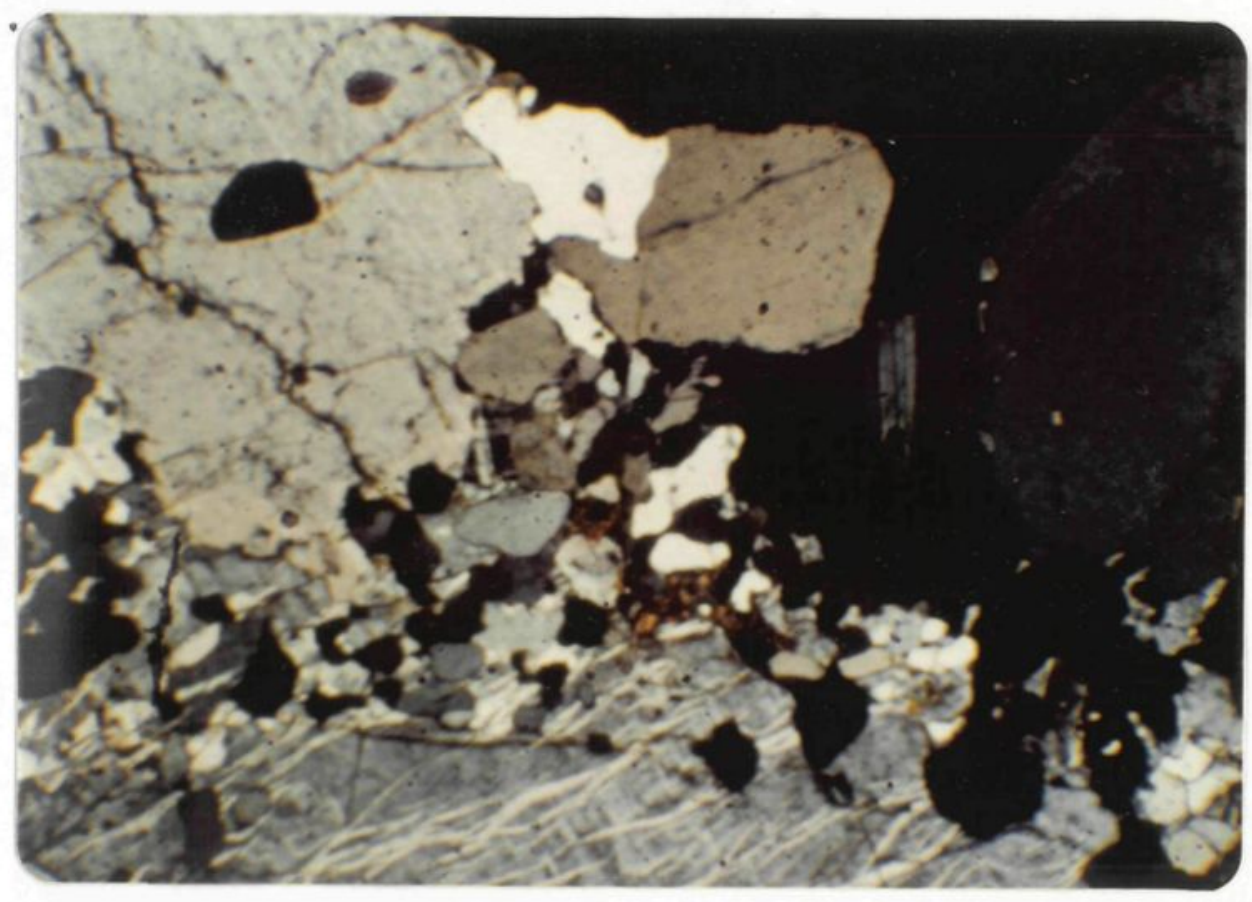

Planche 24: Microphotographie illustrant les changements de l'assemblage minéralogique des pegmatites faiblement métasomatisées. Nous observons l'aégyrine qui ceinture les grains de quartz et qui remplace les minéraux à grains fins. Nous notons l'augmentation de la phase exsolvée des grains de microcline. $\varnothing=6.5 \mathrm{~mm}$ 


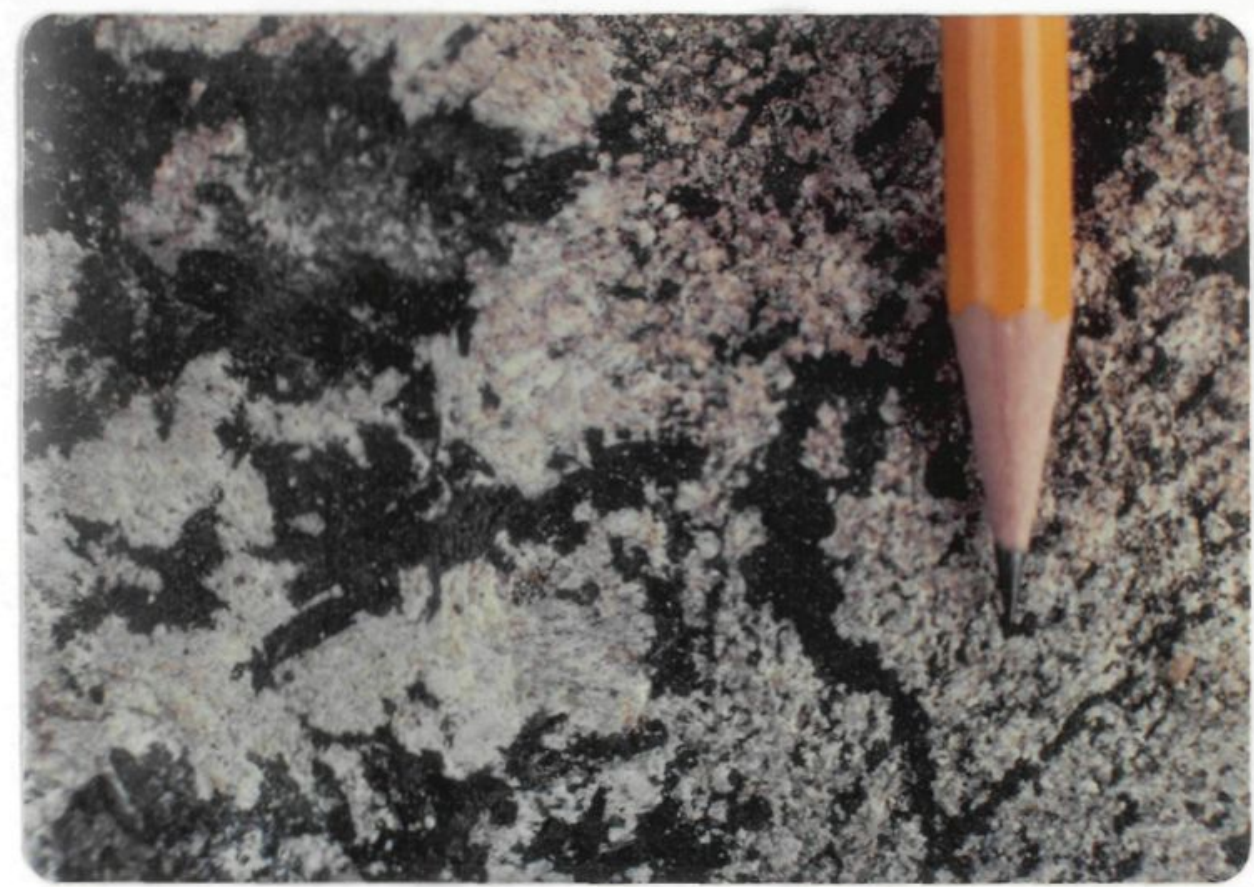

Planche 25: Photographie illustrant 1'aspect des pegmatites métasomatisées. L'aégyrine a remplacé complètement le quartz, nous notons la présence de veinules et d'aggrégats d'aégirine.

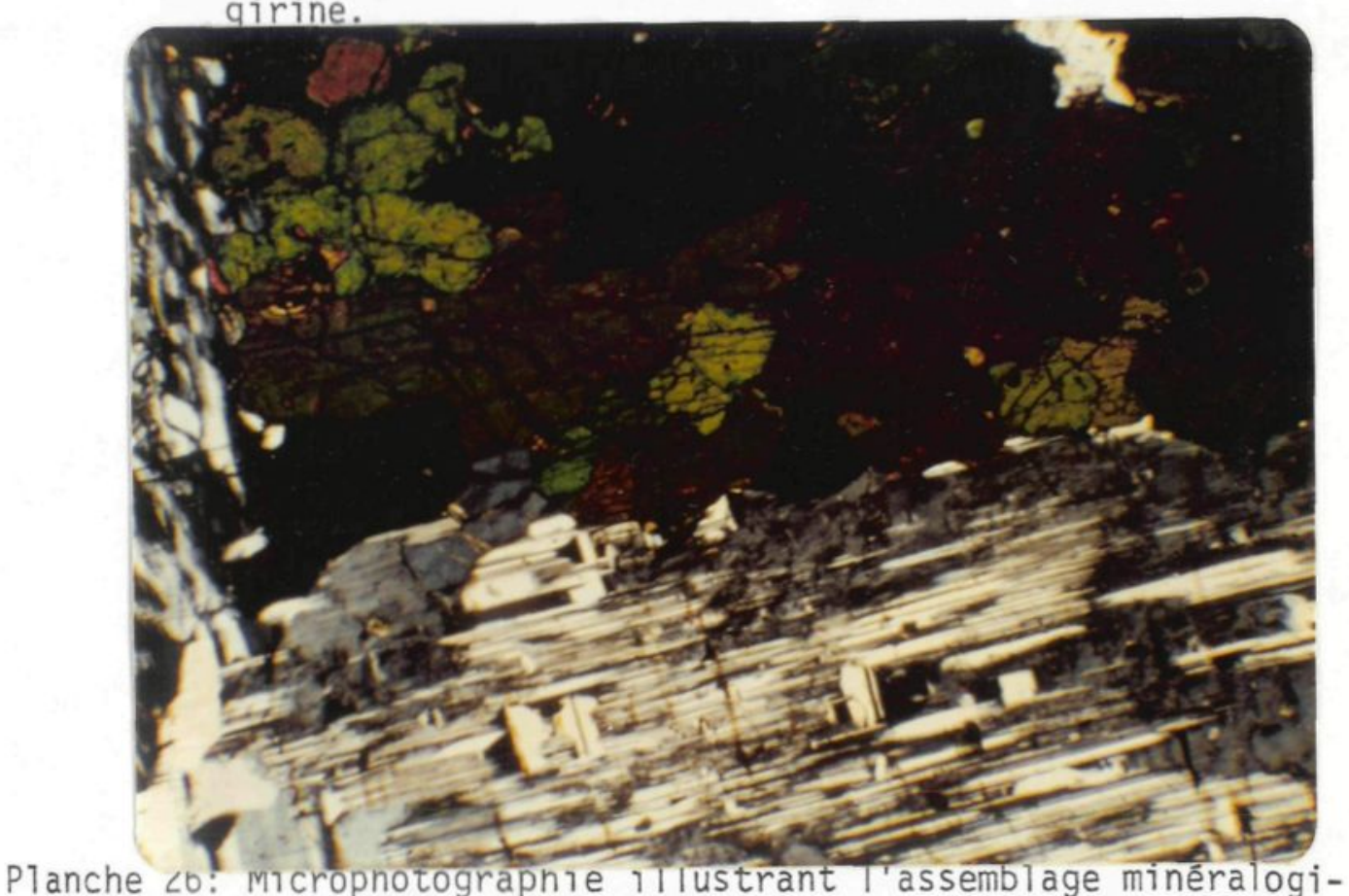
que des pegmatites métasomatisées. L'aégyrine a remplacé le quartz, la biotite et les feldspaths alcalins à grains fins; et commence a remplacer les gros cristaux de microcline. La phase exsolvée de ces derniers a considérablement augmentée. $\varnothing=6.5 \mathrm{~mm}$ 


\section{GEOCHIMIE DES ROCHES DU COMPLEXE DE CREVIER}

Cette partie de l'étude du complexe de Crevier n'a pas pour but d'expliquer l'origine du complexe, mais de fournir quelques données de base sur les principales roches qui composent le massif et qui pourront servir pour des études ultêrieures plus détaillées.

Nous avons analysé 27 échantilions qui sont groupēs selon le type de roches, soit: les monzosyénites à néphēline I, II, III et IV (21); les diorites à biotite (3) et les monzosyénites (2). Les analyses et les calcuis de la norme C.P.I.W. sont disponibles dans l'annexe VII . Les monzosyénites à néphéline ont été groupées en un seul bloc, car suite à une première évaluation des résultats, ces roches ont montré un comportement chimique commun, sans aucune distinction entre les types de monzosyénites à néphëline définis précédemment.

Les diagrammes pétrochimiques utilisés sont le diagramme A-F$M\left(\mathrm{Na}_{2} \mathrm{O}-\mathrm{K}_{2} \mathrm{O}\right.$, Fer total, $\left.\mathrm{Mg}_{2} \mathrm{O}\right)$, Te diagramme $\mathrm{C}-\mathrm{N}-\mathrm{K}\left(\mathrm{CaO}, \mathrm{Na}_{2} \mathrm{O}, \mathrm{K}_{2} \mathrm{O}\right)$ et la combinaison des diagrammes QZ-NE-KS (quartz, néphéline et kalsilites) et QZ-AN-KS (quartz, anorthite et kalsilites), combinaison utilisée dans le but de visualiser les données de manière tridimensionelle.

\subsection{Présentation des résultats}

a) Le_diagramme_A-F-M

Le diagramme A-F-M (figure 6) permet d'identifier une légère différenciation des roches du complexe, illustrée par la concentration de 
FIGURE 6

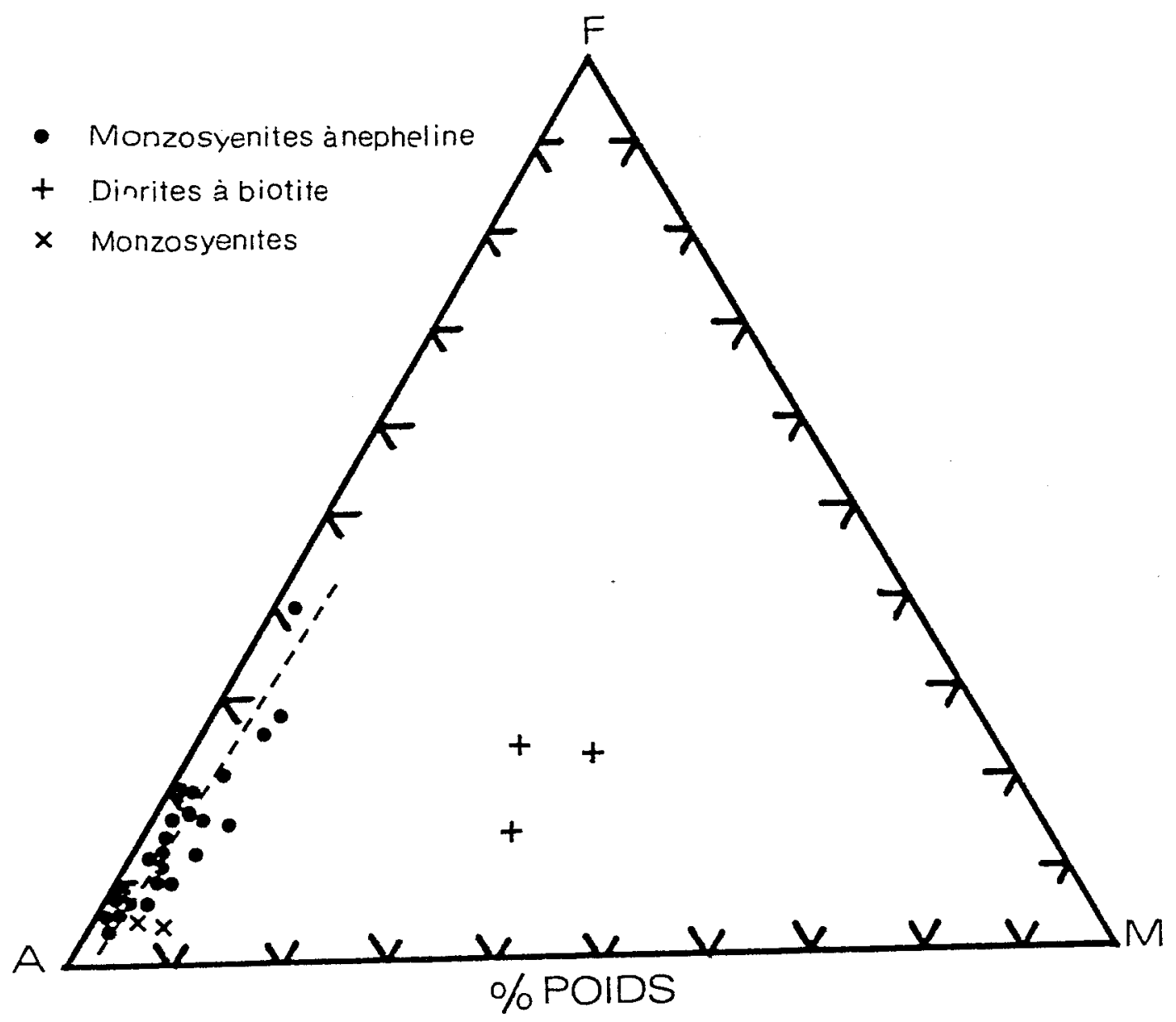

Diagrámme AFM des roches du complexe de Crevier 
points dans la région du pôle $A$ et le long de l'axe A-F. Sur cet axe nous observons quelques (3) échantilions qui sont nettement plus rapprochés du pôle F. La position de ces points correspond aux monzosyénites à néphéline et biotite III, la dispersion vers le pôle $F$ peut être expliquêe par leur contenu plus êlevé en biotite et par conséquent plus élevé en fer, par rapport aux autres types monzosyénites à néphéline. La position isolée des trois diorites à biotite indique que le comportement de ce type de roche ne peut être relié à celui des monzosyénites à néphêline et des monzosyénites.

\section{b) - Le_diagramme $C-N-\underline{N}-\underline{K}$}

Le diagramme $\mathrm{C}-\mathrm{N}-\mathrm{K}$ (figure 7 ) indique une dispersion de la majorité des échantilions le long d'un axe représenté par la ligne en pointillé. Le rapport $\mathrm{Na} / \mathrm{K}$ reste assez constant. Bien que le positionnement des échantillons indique une différenciation, nous croyons qu'elle est exagérée par le contenu variable en calcite des roches, masquant le comportement initial du calcium. Il est fort probable qu'en retranchant le $\mathrm{CaO}$ et le $\mathrm{CO}_{2}$ nécessaire à la formation de la calcite, cela aurait pour effet de raccourcir l'axe de différenciation, le ramenant a une grandeur de l'ordre de celui illustré dans le diagramme précédent.

Nous retrouvons la position isolée des diorites à biotite par rapport aux autres types de roches. Le contenu en calcium des plagioclases (An 30), qui composent la roche, est plus élevé que celui des monzosyénites (An 7) ce qui résulte à la position plus rapprochẻe du pôle $\mathrm{CN}$. Notons que la remarque faite au sujet du contenu en calcite des autres roches, s'applique également aux diorites à biotite. . 
FIGURE 7

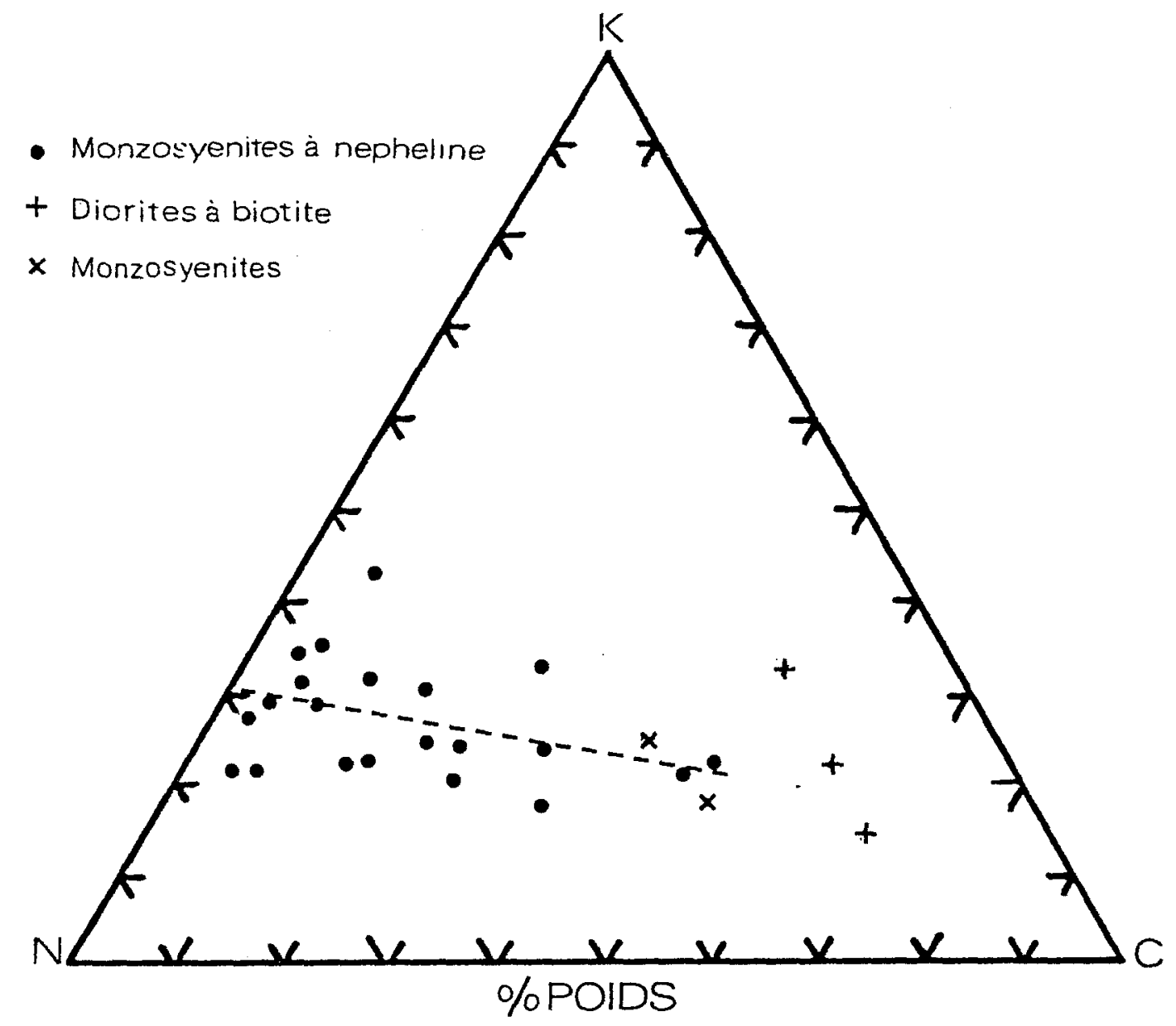

Diagramme CNK des roches du complexe de Crevier 
c) - Les diagrammes QZ-NE-KS et $0 Z-A N-K S$

Le diagramme QZ-NE-KS (figure 8) est utilisé avec le diagramme QZ-AN-KS (figure 9) dans le but de visualiser les roches du complexe sous la forme d'un tétraède ayant pour base le plan QZ-NE-KS et pour sommet le pôte AN. La combinaison des deux diagrammes indique une faible différenciation des roches du complexe de Crevier. Elle est marquée par la perte d'anorthotite (figure 9), pour aboutir à une concentration de points au point OR (orthose) et sous le point $M^{\prime \prime}$, point eutectique ternaire des syênites à néphéline à la pression de $6 \mathrm{~kb}$ (Roux et Hamilton, 1976), sur le diagramme QZ-NE-KS (figure 8).

Nous avons tenté d'évaluer la pression $\left(\mathrm{P}_{\mathrm{H}_{2} \mathrm{O}}\right)$ à laquelle le massif s'est mis en place. Nous avons indiqué sur le diagramme QZ-NE-KS (figure 8) les domaines de stabilité de la néphéline et de la leucite (Hamilton et Mackenzie,1965) ainsi que les points eutectiques ternaires $M\left(P_{\mathrm{H}_{2} \mathrm{O}}=1 \mathrm{~atm}\right.$. $) M^{\prime}\left(P_{\mathrm{H}_{2} \mathrm{O}}=1 \mathrm{~kb}\right)$ et $M^{\prime \prime}\left(\mathrm{P}_{\mathrm{H}_{2} \mathrm{O}}=6 \mathrm{~kb}\right)$, d'après les travaux de Bowen (1958), Hamilton et Mackenzie (1965) et Roux et Hamitton (1976). Nous constatons que le point eutectique ternaire a tendance à s'abaisser vers le pôle NE, de la même façon que le point eutectique ternaire des granites s'abaisse vers le pôle $A B$ (albite) du système QZ-ABOR (Luth, Jahns et Tuttle, 1964) entre les pressions $0.5 \mathrm{~kb}$ et $10 \mathrm{~kb}$. Nous pouvans suggérer que le point eutectique ternaire des syénites à néphēline, a le même comportement que celui des granites sur de telles intervalles de pression. Suite à ces constatations nous avons comparé, sur le diagramme QZ-NE-KS, le positionnement des points eutectiques ternaires $M$, $M^{\prime}$ et $M^{\prime \prime}$ avec le groupement d'échantilions situé sous le point $M$ ". Ce groupement correspond en général aux échantilions les plus différenciés $(A n=0)$, 
- Monzosyénites à nepheline

$\times$ Monzosyenites

+ Diorıtes à bıotite

- Minımum

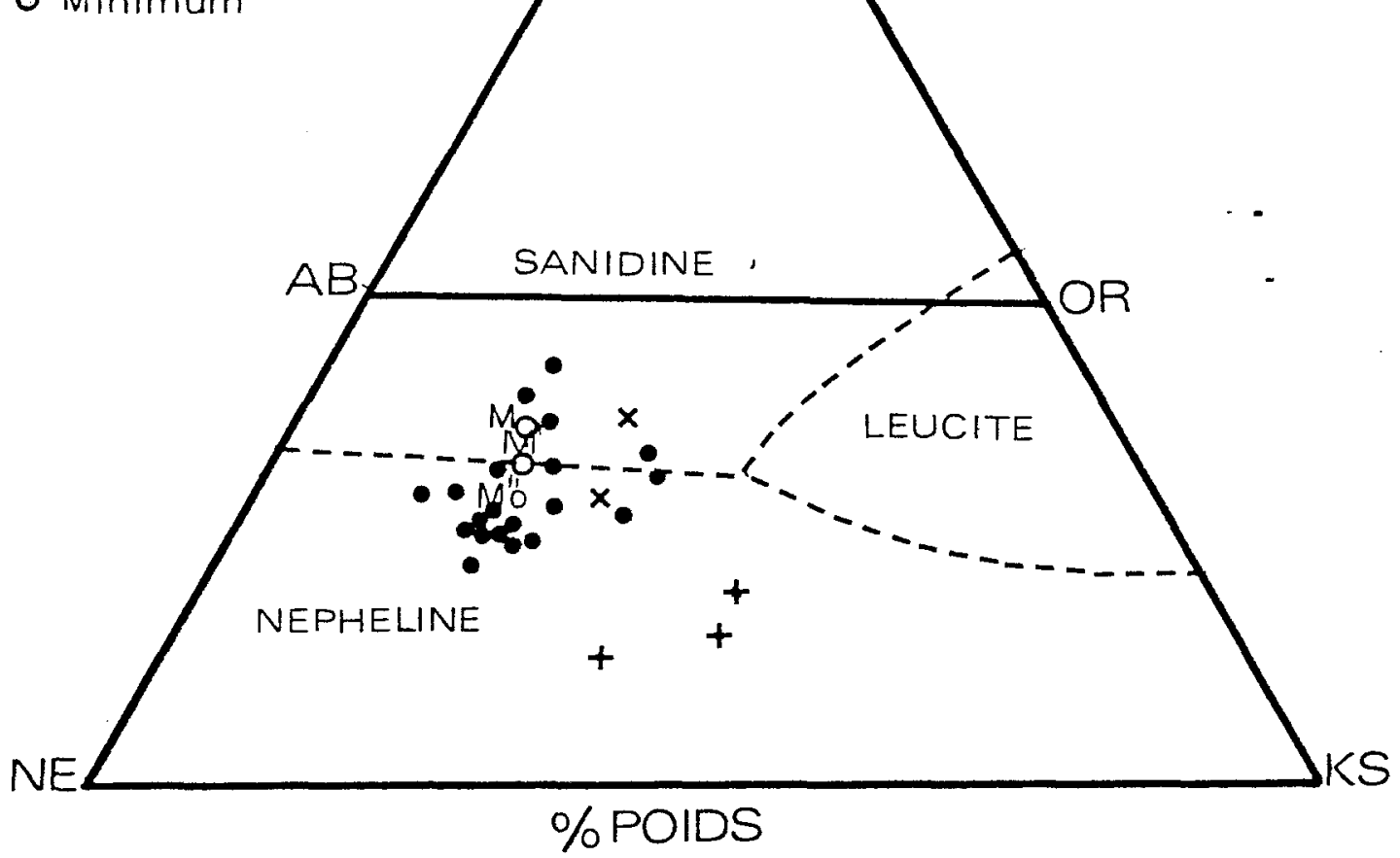

Diagramme QZ-NE-KS montrant I'évolution des roches du comlexe de Crevier(d'après Hamilton et Mackensie(1965)) 


\section{FIGURE 9}

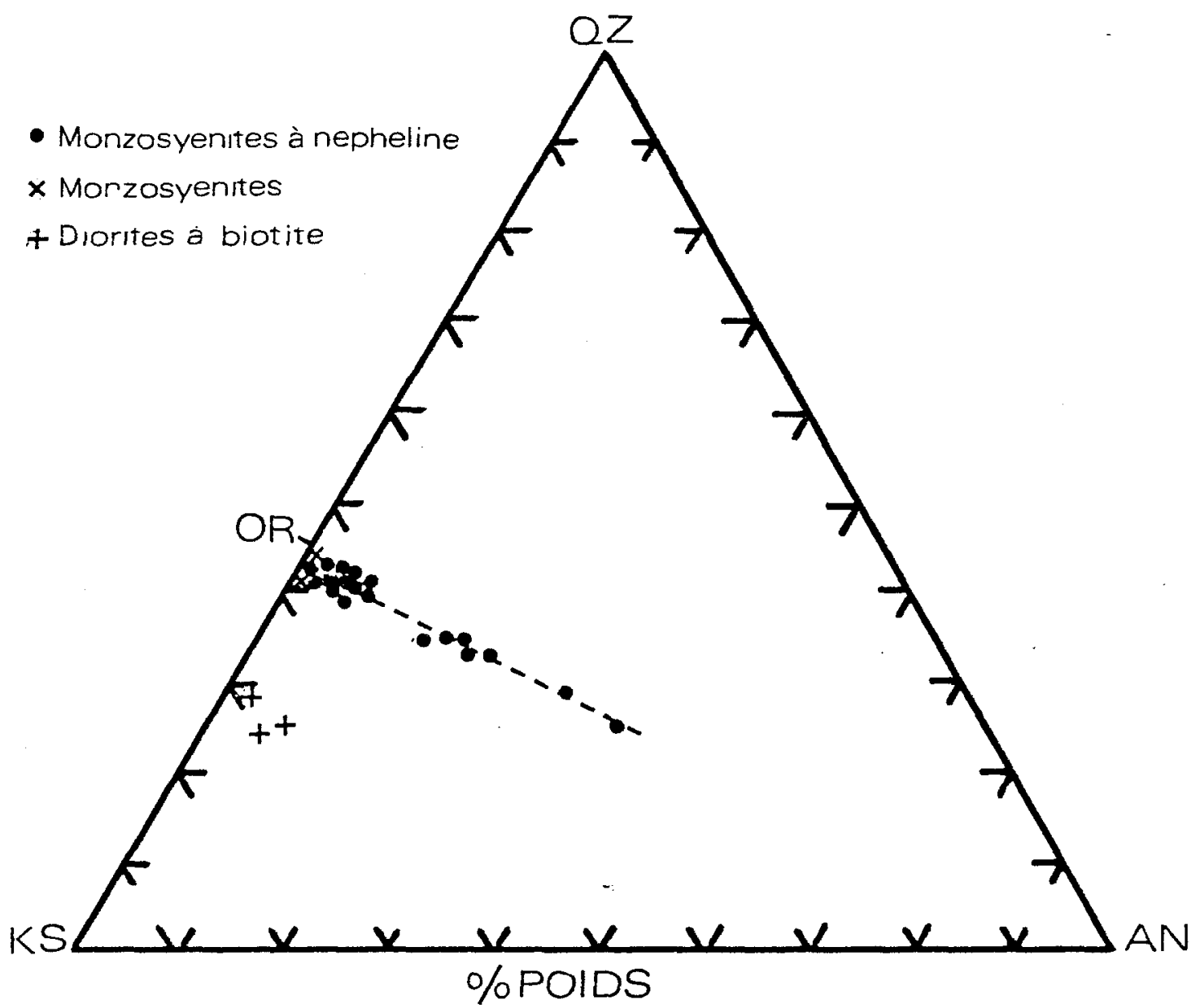

Diagramme QZ-KS-AN illustant l'évolution des roches du complexe de Crevier 
I1s représenteraient un minimum eutectique ternaire qui correspondrait à une pression de vapeur d'eau de 7-8 kb $\left(M^{\prime \prime}=6 \mathrm{~Kb}\right)$. Naturellement cette pression de mise en plan des roches du complexe est hypothétique, des études plus détaillées seraient nécessaire pour confirmer ces valeurs, telles que l'étude des inclusions fluides.

Notons que les diorites à biotite ne montrent, sur le diagramme QZ-NE-KS, aucune affinité avec les monzosyénites et les monzosyēnites à néphêline.

L'examen des diagrammes A-F-M, C-N-K, QZ-NE-KS et QZ-AN-KS indique une différenciation des monzosyēnites et des monzosyénites à néphéTine du complexe, à une profondeur estimée à $40 \mathrm{~km}(7 \mathrm{~kb})$. Il est difficile de conclure de façon certaine sur le mode d'origine des roches du complexe. Les différents types de roches observés ne montrent aucune relation spatiale avec des membres basiques, du moins au niveau d'érosion actuel du complexe. Nous pouvons émettre trois hypothèses quant à cette origine:

1) - Cristallisation fractionnée au niveau du manteau

2) - Fusion partielle d'un matériel approprié au-niveau de la croute inferieure.

3) - Métasomatisme de la roche (gneiss) in-situ

Nous pouvons éliminer la troisième hypothèse, car les relations du contact pluton - encajssant, décrits dans le chapitre précédent ainsi que les relations des structures du massif et de l'encaissant, ont mis en évidence le caractère intrusif du complexe de crevier. Quant aux deux autres hypothèse, soit la cristallisation fractionnée et la fusion partielle d'un matériel approprié, on ne peut les départager à l'aide des diagrammes pétrochi- 
miques qui sont présentés, car les deux modes d'origine des roches du complexe que nous proposons peuvent être illustrés par les résultats que nous avons obtenu sur les diagrammes OZ-NE-KS et QZ-AN-KS. L'absence de membres basiques peut être interprētée de deux façons. La première est qu'ils se situent a un niveau plus profond par rapport au niveau actuel d'érosion et que le complexe peut être issu d'une différenciation fractionnēe au niveau du manteau. La deuxième est que l'absence de ces membres basiques est du a une fusion partielle au niveau de la croute inférieure suivi d'une cristaliisation fractionnée. Des études sur la composition isotopique du strontium (Be17 et Powe11,1971) pourraient lever le doute en incluant la source du magma (manteau supérieur ou croute inférieure) et par le fait même infirmer ou confirmer l'une des deux hypothèse proposées.

L'origine des diorites à biotite n'est pas établie avec certitude. L'étude pétrochimique montre que ce type de roche possède un comportement différent des monzosyẹnites et des monzosyénites à néphẹline. D'après les textures observées sur le terrain (unité 1), la mise en place de la diorite à biotite est comtemporaine aux monzosyénites à néphéline. I. Les textures de "distension" observées dans ces mêmes roches ont été reliées à une mise en place sous des conditions d'extension. Il serait donc possible que les diorites à biotite soient des reliques de l'encaissant suite à la mise en place de la monzosyénite à néphéline I, sous la forme d'injections lit-parlit dans une zone d'extension. Ce mode d'origine est compatible avec le modèle de contrôle structural qui a été proposé pour expliquer le fort rubannement de 7 'unité I, ainsi que les textures de recristallisation des plagioclases (oligoclase) qui composent cette roche (cf. chap. I). 
CHAPITRE IV

\section{GEOCHIMIE DES FENITES}

La gêochimie des fénites de Crevier a été évaluée en se servant des analyses totales des dykes de pegmatitepost-tectonique qui traversent complètement la zone métasomatisée. (La méthode d'échantillonnage, la préparation des échantilions ainsi que les méthodes d'analyse sont disponibles dans les annexes I à III). Bien que les effets texturaux du métasomatisme diffèrent selon le type de roche, nous tenterons de proposer un modēle global des effets du métasomatisme à l'aide des pegmatites; elles ont êté choisies pour leur homogênéité texturale et minéralogique. Nous avons estimé leur fénitisation sous deux aspects: un aspect qualitatif, à l'aide de diagramme pétrochimique et un aspect quantitatif à l'aide de 7 'équation de Gresens (1967). Cette équation permet l'étude des relations composition-volume des transferts de masse lors du processus de fénitisation.

\section{1 - Evaluation qualitative du métasomatisme}

Comme il a été dit précédemment, cette évaluation se veut une évaluation qualitative des fénites de Crevier. Nous avons effectué des calculs de la norme C.P.I.W., provenant d'analyses totales de pegmatites post-orogeniques du Grenville échantillonnées le long d'une coupe, qui traverse complètement la zone de fénites de l'unité 4. (Les analyses et les calculs de la norme sont disponibles dans 1'annexe 8). Les résultats 
sont compilés sur des diagrammes pétrochimiques.

Les diagrammes retenus pour cette ëtude sont:

le diagramme A-F-M, le diagramme $\mathrm{C}-\mathrm{N}-\mathrm{K}$ et le diagramme QZ-NE-KS.

\section{1-1 Interprétation des diagrammes pétrochimiques}

a) - Le diagramme A-F-M

Le diagramme A-F-M (figure 10) permet d'individualiser deux groupes de roches. Un groupement près du pôte A, représentant les pegmatites non métasomatisées qui sont loin du contact de l'intrusion et un groupement qui se concentre vers le pôle $F$ représentant les roches métasomatisēes échantillonnées près du contact de l'intrusion. Nous attribuons le déplacement des pegmatites le long de T'axe A-F (1igne pointillée dans la direction représentée par la flèche) à un enrichissement substantiel de la teneur en fer dans la roche, attribuée à la présence d'aégyrine dans les pegmatites métasomatisées et un faible enrichissement en magnēsium.

\section{b) - Le diagramme $\mathrm{C}-\mathrm{N}-\mathrm{K}$}

Le diagramme $\mathrm{C}-\mathrm{N}-\mathrm{K}$ (figure 11) montre une évolution des pegmatites représentée par une perte substantiẹtle en potassium $\left(K_{2} 0\right)$ jumelée à des gains de $\mathrm{Na}_{2} \mathrm{O}$ (aégyrine) et CaO (calcite). La faible pente vers le pôle $C$ exprime bien les observations faites relatives à l'augmentation de calcite lorsqu'on s'approche du contact du massif alcalin. 
FIGURE 10

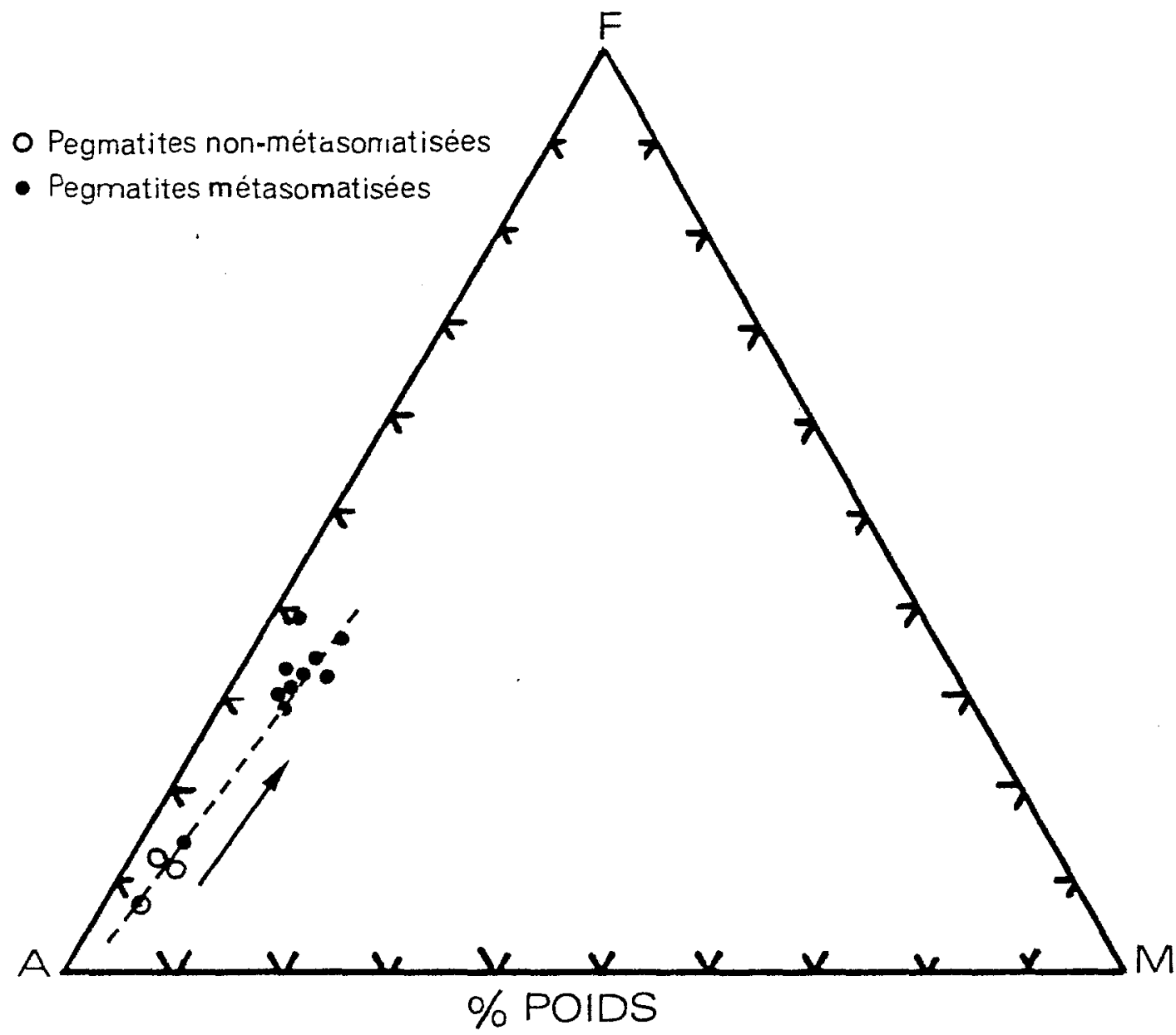

Diagramme AFM des pegmatites del'encaissant du complexe de Crevier 
FIGURE 11

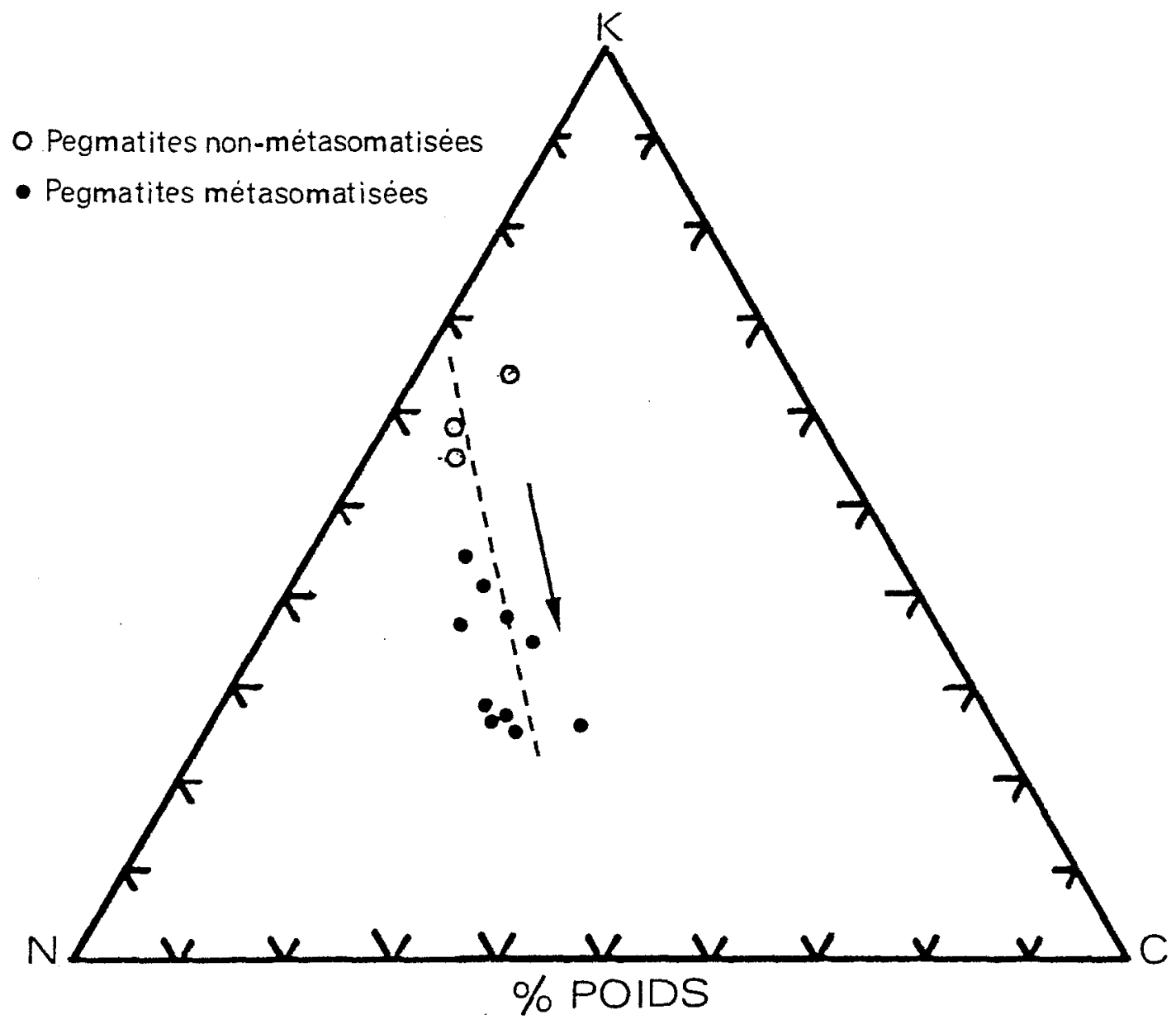

Diagramme CNK des pegmatites de l'encaissant du complexe de Crevier 


\section{c) - Le diagramme $Q Z Z-N E-K S$}

Le diagramme QZ-NE-KS (figure 12) peut être apparenté, comme celui utilisé dans le chapitre précēdent, au système combiné des granites et des roches sous-saturées. Le positionnement des pegmatites illustre, une désilification de la roche métasomatisé. Nous observons trois échantillons dans le domaine des granites ( $Q Z-A B-O R)$, représentant les pegmatites non-mëtasomatisées. Les autres échantilions se situent sur la barrière thermique $A B-O R$ et au-delà, dans le domaine des roches sous-saturées. Cette évolution gëochimique pourrait être comparée à une transformation à l'état solide des roches (pegmatites). Le produit de départ est une roche de composition "granitique" et le produit final, est une roche soussaturēe apparentée à une syénite à néphēline.

En résumé, l'étude des diagrammes pétrochimiques indiquent l'évolution de certains élēments dans le passage d'une pegmatite non-métasomatisée, à une pegmatite métasomatisée, soit:

- Un enrichissement en $\mathrm{CaO}, \mathrm{Na}_{2} \mathrm{O}, \mathrm{MgO}$ et Fe total

- Une perte en $\mathrm{SiO}_{2}$ et en $\mathrm{K}_{2} \mathrm{O}$

L'évaluation quantitative nous permettra de vérifier ces affirmations et de les quantifier.

\section{2 - Evaluation quantitative du métasomatisme}

Les estimés quantitatifs de transferts de masse, ont gënéralement été calculés à l'aide d'une cellule standard de 100 anions (Barth, 
FIGURE 12

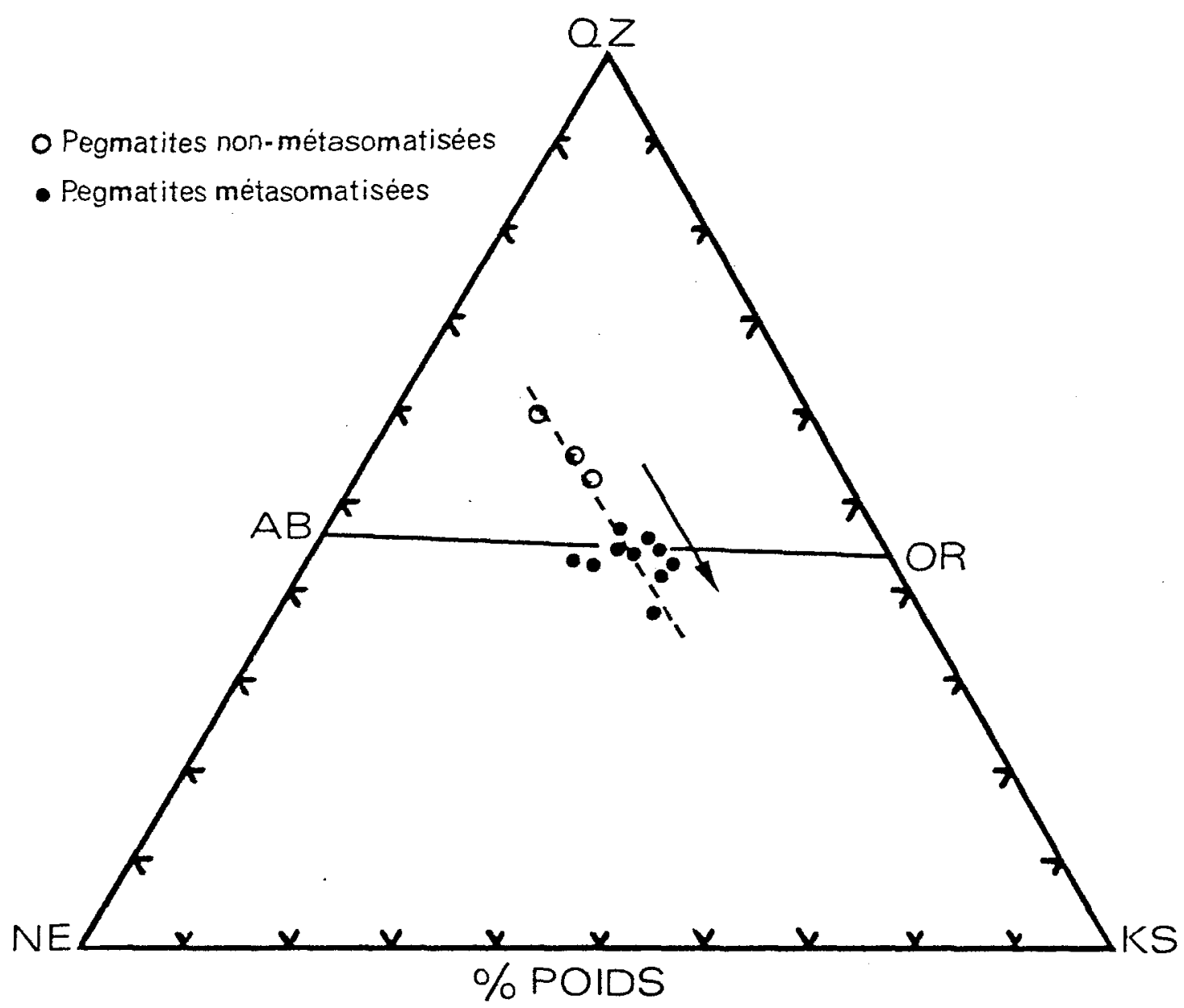

Diagramme QZ-NE-KS des pegmatites de l'encaissant du complexe de Crevier 
1948) ou une cellule à volume standard (Bogolepov, 1963). Ces méthodes de calcul de gains et des pertes d'épéments requièrent des conditions spécifiques, telles que le poids spécifique du matériel constant et le volume de roche altērée et non-altérée constant. Gresens (1967) a mis au point une équation qui fait la relation entre les échanges de matière et les variations volume-composition de la roche. L'équation de base de Gresens (1967) est la suivante:

$$
x_{n}=a\left[\left(F_{V} c_{n}^{\beta} \frac{\rho^{\beta}}{\rho \alpha}\right)-c_{n}^{\alpha}\right]
$$

où:

$X_{n}=$ Gains et pertes de l'élément " $n$ " dans la production de la roche $\beta$ à partir de la roche $\alpha$

$a$ = Quantité initiale de matière, désigné généralement comme 100 , amenant la valeur de $x_{n}$ en $\%$ de poids.

$F_{v}=$ Facteur volume, lorsque $F_{v}=1$ nous sommes en présence d'un remplacement volume par volume. Si $F_{v}<1$, nous avons une perte de volume. Si $F_{V}>1$, nous avons une augmentation de volume.

$c_{n}^{\alpha \beta}=$ Fraction de poids de la composante " $n$ " de la roche $\alpha$ (roche parentale) et de la roche $\beta$ (roche métasomatisée) = Poids spécifique de la roche utilisée. 
Plusieurs auteurs ont utilisés cette méthode de calcul tels que Appleyard et Woolley (1978) et Kerrich et Al (1977). Ils ont mis en évidence le rôle du facteur volume et du poids spécifique dans les calcuis de transfert de masse. Par comparaison avec la méthode de Barth (1948), ils ont évalué une différence de 10\% dans les résultats lorsque l'on ignore le poids spécifique et une erreur pouvant aller jusqu'à $20 \%$, lorsque le facteur volume est ignoré. La méthode de calcul que nous utiliserons sera celle de Gresens (1967), à T'aide de T'équation de base mentionnẻe précédemment.

\section{2-1 Méthode de calcul}

L'équation de base proposée par Gresens (1967) est une équation à deux inconnues, soit $X_{n}$ et $F_{v}$. Pour la résoudre nous devons lever une des deux inconnues, soit $F_{v}$. L'évaluation du facteur volume peut se faire de deux façons, soit de présumer que $F_{V}=1$, c'est à dire que le métasomatisme s'est effectué volume par volume. D'après les observations pétrochimiques tel que les changements texturaux de la roche et la fracturation des feldspaths alcalins, l'hypothèse d'un remplacement volume par volume doit être rejetée. La deuxième possibilité est d'évaluer la variation de volume de chaque roche en fonction de la roche parentale et ce graphiquement. Il s'agit de tracer un diagramme composition-volume $(C-V)$ de chaque roche, contenant les courbes de l'équation de base de chaque élément, en assumant plusieurs valeurs de $F_{v}$ et le $x_{n}$ correspondant. 
Les diagrammes $\mathrm{C}-\mathrm{V}$ étant construits, nous pouvons déterminer la valeur de $F_{v}$ correspondant à $X=0$ de plusieurs courbes d'une même roche et ce pour tous les échantilions, interceptant la valeur $x_{n}=0$ au même endroit sur 7'abcisse. Nous interprétons ce ou ces êléments comme étant relativement immobile en regard de leur taux de variation dans les analyses. (voir un exemple de calcul dans l'annexe VI).

La valeur de $F_{v}$ étant assumée, il est facile de déterminer à partir de l'équation de base, la valeur de $x_{n}$ pour chaque élément d'un échantillon et enfin de tracer un diagramme global des gains et des pertes en fonction de la position des échantillons le long de la coupe effectuée sur le terrain.

\section{2-2 Présentation des données.}

Les analyses chimiques utilisées pour cette étude sont disponibles dans l'annexe VII. Diverses modifications des résultats ont été effectués. Le fer total a été recalculé sous la forme de $\mathrm{Fe}_{2} \mathrm{O}_{3}$ par la relation $\left.\mathrm{Fe}_{t}\left(\mathrm{Fe}_{2} \mathrm{O}_{3}\right)=\left[(\% \mathrm{FeO} \times 1.11) \div \% \mathrm{Fe}_{2} \mathrm{O}_{3}\right)\right]$. Le calcium a été réévalué en tenant compte de la présence de calcite dans les pegmatites. La calcite peut provenir à la fois des émanations qui ont provoqué le métasomatisme I et de l'intrusion de la carbonatite. Les deux événements n'ont aucune relation entre eux (cf. chap. 1). Il est impossible d'évaluer quantitativement lequel des deux événements a produit la calcite dans les roches de l'encaissant. Nous avons retiré des analyses, les quantités nécessaires de $\mathrm{CaO}$ et $\mathrm{CO}_{2}$ pour former la calcite, en assumant que tout le $\mathrm{CO}_{2}$ provient de l'intrusion de la carbonatite. La dernière manipulation est 1 a recalculation à $100 \%$ des données de l'analyse chimique totale sur une base anhydre. Les analyses recalculées sont présentées dans le tabieau 3. 
ANALYSES MODIFIEES POUR L'ETUDE GEOCHIMIQUE DES PEGMATITES (METASOMATISEES ET NON-METASOMATISEES)

\begin{tabular}{|c|c|c|c|c|c|c|c|c|c|c|c|c|c|c|}
\hline $\mathrm{SiO}_{2}$ & $\begin{array}{c}\text { I } \\
60.88\end{array}$ & $60^{2} .89$ & $\begin{array}{c}3 \\
61.43\end{array}$ & $6 I^{4} .16$ & $\begin{array}{c}5 \\
60.69\end{array}$ & $\begin{array}{c}6 \\
62.20\end{array}$ & $\begin{array}{c}7 \\
61.60\end{array}$ & $\stackrel{8}{6 I .60}$ & $\begin{array}{c}9 \\
63.80\end{array}$ & $\begin{array}{c}10 \\
65.90\end{array}$ & $\begin{array}{c}\text { II } \\
71.80\end{array}$ & $\begin{array}{c}\mathrm{I} 2 \\
7 \mathrm{I} .80\end{array}$ & $\begin{array}{c}13 \\
69.60\end{array}$ & $\begin{array}{c}\mathrm{I} 4 \\
7 \mathrm{I} .06\end{array}$ \\
\hline $\mathrm{Al}_{2} \mathrm{O}_{3}$ & I3.83 & I3.42 & I 3.42 & 14.32 & I2. 80 & 14.49 & I3. 80 & 13.90 & 13.70 & I6. 70 & 14.50 & $\mathrm{I} 5.2 \mathrm{I}$ & 15.40 & I5.03 \\
\hline $\mathrm{Fe}_{\mathrm{T}}$ & 8.07 & 8. I6 & $7.3 I$ & 6.60 & $8.5 \mathrm{I}$ & 6.67 & 7.25 & $7.4 \mathrm{I}$ & 5.95 & 2.28 & I. 20 & I. 7I & 2.22 & I. 7I \\
\hline $\mathrm{CaO}$ & 2.93 & 3.08 & 3.47 & 2.76 & 3.39 & 2.83 & 2.82 & 2.50 & 3.28 & I. I6 & 0.57 & 0.74 & 0.76 & 0.69 \\
\hline $\mathrm{Na}_{2} \mathrm{O}$ & 7.48 & 7.79 & 7.22 & 7.86 & $7 . I 5$ & 6.58 & 6.34 & 6.28 & 5.68 & 6.26 & 4.30 & 2.73 & 4.32 & 3.78 \\
\hline $\mathrm{TiO}_{2}$ & 0.75 & 0.43 & 0.59 & 0.34 & 0.54 & 0.40 & 0.37 & $0.4 I$ & 0.40 & $0.2 \mathrm{I}$ & 0.20 & 0.25 & 0.30 & 0.25 \\
\hline $\mathrm{P}_{2} \mathrm{O}_{5}$ & $0.4 \mathrm{I}$ & 0.46 & 0.41 & 0.49 & I. 30 & 0.36 & 0.42 & $0.4 \mathrm{I}$ & 0.28 & 0.33 & 0.06 & 0.04 & 0.03 & 0.04 \\
\hline $\mathrm{MnO}$ & 0.12 & 0.15 & 0.17 & 0.19 & 0.19 & 0.16 & 0.24 & 0.15 & 0.25 & 0.10 & 0.03 & 0.02 & 0.02 & 0.03 \\
\hline TOTAL: & 99.94 & 99.98 & I00 & 100 & 99.98 & 100 & 100 & 100 & I00 & I00. I & 99.97 & 99.93 & 99.96 & 100 \\
\hline
\end{tabular}


Les roches métasomatisées sont reprêsentées par les analyses \# 1 à \# 10 inclusivement (métasomatisme décroissant) et la roche parentale est représentée par l'analyse \#14 qui est obtenue par le calcul de la moyenne de chaque oxyde des analyses \# 11, 12 et 13, composées de pegmatites non métasomatisēes. (L'échantilion \# 11 a été pris en fonction de 1 a coupe effectuée sur le terrain et les échantilions \# 12 et 13 ont été pris alēatoirement, en dehors de l'influence du complexe igné) (voir figure 19).

\section{2-3 Présentation des résultats}

a) Détermination de $F_{-}$

Comme il a été dit précēdemment, nous avons calculé a partir des analyses, le taux de variations maximum de chaque élément des roches métasomatisées par rapport à la roche parentale. Les résultats sont les suivants:

$\begin{array}{ll}\mathrm{SiO}_{2} & 15 \% \\ \mathrm{Al}_{2} \mathrm{O}_{3} & 13 \% \\ \mathrm{Fe}_{2} \mathrm{O}_{3} & 80 \% \\ \mathrm{MgO} & 70 \% \\ \mathrm{CaO} \mathrm{O} & 79 \% \\ \mathrm{Na}_{2} \mathrm{O} & 52 \% \\ \mathrm{~K}_{2} \mathrm{O} & 37 \% \\ \mathrm{TiO}_{2} & 33 \% \\ \mathrm{P}_{2} \mathrm{O}_{5} & 95 \% \\ \mathrm{MnO} & 95 \%\end{array}$

TABLEAU 4: Taux de variation maximum des éléments dans les roches métasomatisêes. 
On peut présumer, à l'aide de ces calculs que la valeur de $F_{v}$ réelle nous sera donnée par la valeur de $F_{v} a ̀ X_{n}=0$ des éléments Si et $A 1$, du à leur faible mobilité par rapport aux autres êléments.

La deuxième étape est la construction des diagrammes $\mathrm{C}-\mathrm{V}$, représentés dans les figures 13 a 18 . Dans ces diagrammes nous observons des valeurs de $F_{v}$ pour $X_{n}=0$ relatjvement stables pour chaque éléments (échantilions 1 à 9). L'échantilion 10 (pegmatite faiblement métasomatisée) montre une tendance de la valeur de $F_{v}$ à $X_{n}=0$ à se diriger vers $F_{V}=1$. L'échantilion 11 (pegmatite non métasomatisée) montre une concentration des valeurs autour de $F_{v}=1$. Maintenant si nous examons les valeurs de $F_{V}$ pour tous les échantilions (voir le tableau 5) nous constatons que les éléments $\mathrm{Mn}, \mathrm{P}, \mathrm{Ti}, \mathrm{K}, \mathrm{Na}, \mathrm{Ca}, \mathrm{Mg}$ et $\mathrm{Fe}_{t}$ ont une valeur de $F_{v}$ très éloignée de $F_{V}=1(0.2$ et 1.5$)$, ce qui représente des variations de volume $-80 \%$ et $+50 \%$. Ces chiffres sont incompatibles avec les observations faites sur le terrain et en laboratoire. Par contre deux éléments, la silice et l'aluminium, ont des valeurs de $F_{V} q u i$ varient entre 1.05 et 1.10 , soit un gain de volume de $10 \%$. Si nous nous référons aux faibles taux de variations de ces éléments et leur valeur constante de $F_{V}$ dans tous les diagrammes $C-V$, il est fort plausiblede les utiliser comme référence pour la détermination de $F_{v}$. Ce dernier est de 1.08 pour $\mathrm{SiO}_{2}$ et de 1.02 pour $\mathrm{Al}_{2} \mathrm{O}_{3}$ (valeurs moyennes), soit une valeur médiane de 1.05 , ce qui implique que le métasomatisme a produit une légère augmentation de volume, de l'ordre de $5 \%$ du volume total de roche. 


\section{TABLEAU 5}

VALEURS DE $F_{V}$ POUR LES DIFFERENTS OXIDES ET TRACES $A x_{n=0}$

$\begin{array}{lllllllllll}\text { I } & 2 & 3 & 4 & 5 & 6 & 7 & 8 & 9 & \text { IO } & \text { II }\end{array}$

Si I.IO I.I3 I.05 I.09 I.I2 I.IO I.IO I.IO

$\begin{array}{llllllllllll}\text { A1 } & \text { I.03 } & \text { I.05 } & \text { I.0I } & 0.99 & \text { I.I2 } & \text { I.04 } & \text { I.04 } & \text { I.03 } & \text { I.I2 } & 0.82 & 0.99\end{array}$

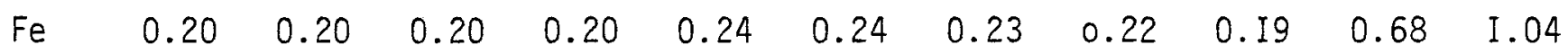

$\begin{array}{llllllllllll}\text { Mg } & 0.25 & 0.45 & 0.4 I & 0.43 & 0.30 & 0.45 & 0.59 & 0.34 & 0.42 & 0.84 & \text { I.IO }\end{array}$

$\begin{array}{llllllllllll}\text { Ca } & 0.40 & 0.20 & 0.19 & 0.43 & 0.19 & 0.24 & 0.25 & 0.24 & 0.23 & 0.49 & 1.00\end{array}$

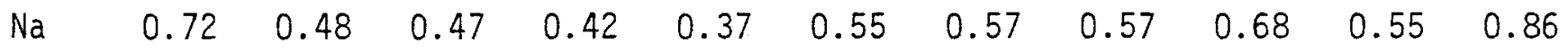

$\begin{array}{lllllllllll}\text { K. I. I.46 } & \text { I.47 } & \text { I.35 } & \text { I.28 } & \text { I.65 } & \text { I.05 } & \text { I.04 } & \text { I.09 } & \text { I.22 } & 0.96 & 0.95\end{array}$

Ti $\quad \begin{array}{lllllllllll}0.50 & 0.60 & 0.42 & 0.75 & 0.65 & 0.60 & 0.70 & 0.62 & 0.60 & 0.95 & \ldots\end{array}$

P $\quad \ldots \quad--\begin{array}{lllllllllll} & 0.20 & 0.42 & \ldots & 0.20 & 0.50 & 0.63 & 0.62 & 0.60 & 0.50 & \ldots\end{array}$

$\begin{array}{llllllllllll}\text { Mn } & 0.80 & 0.68 & 0.42 & 0.42 & 0.65 & 0.52 & 0.63 & 0.57 & 0.58 & 0.95 & \ldots\end{array}$

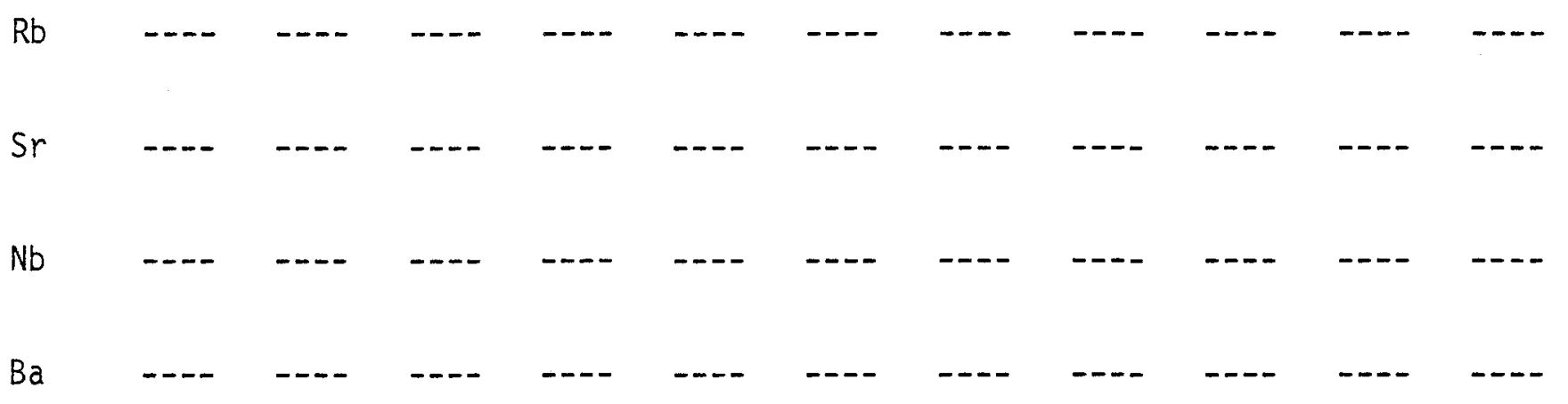




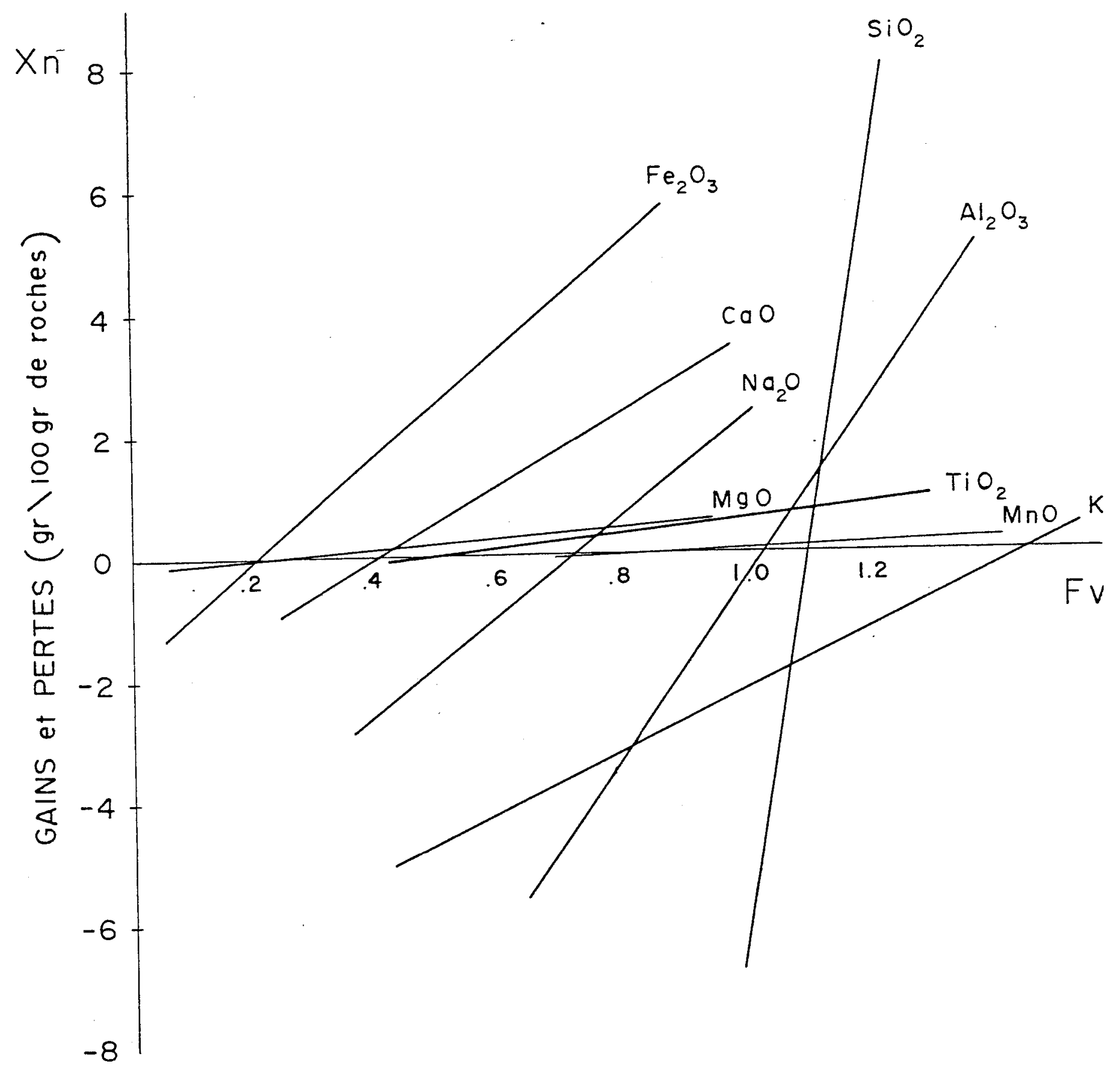

DETERMINATION de FV

Fig.: 13 


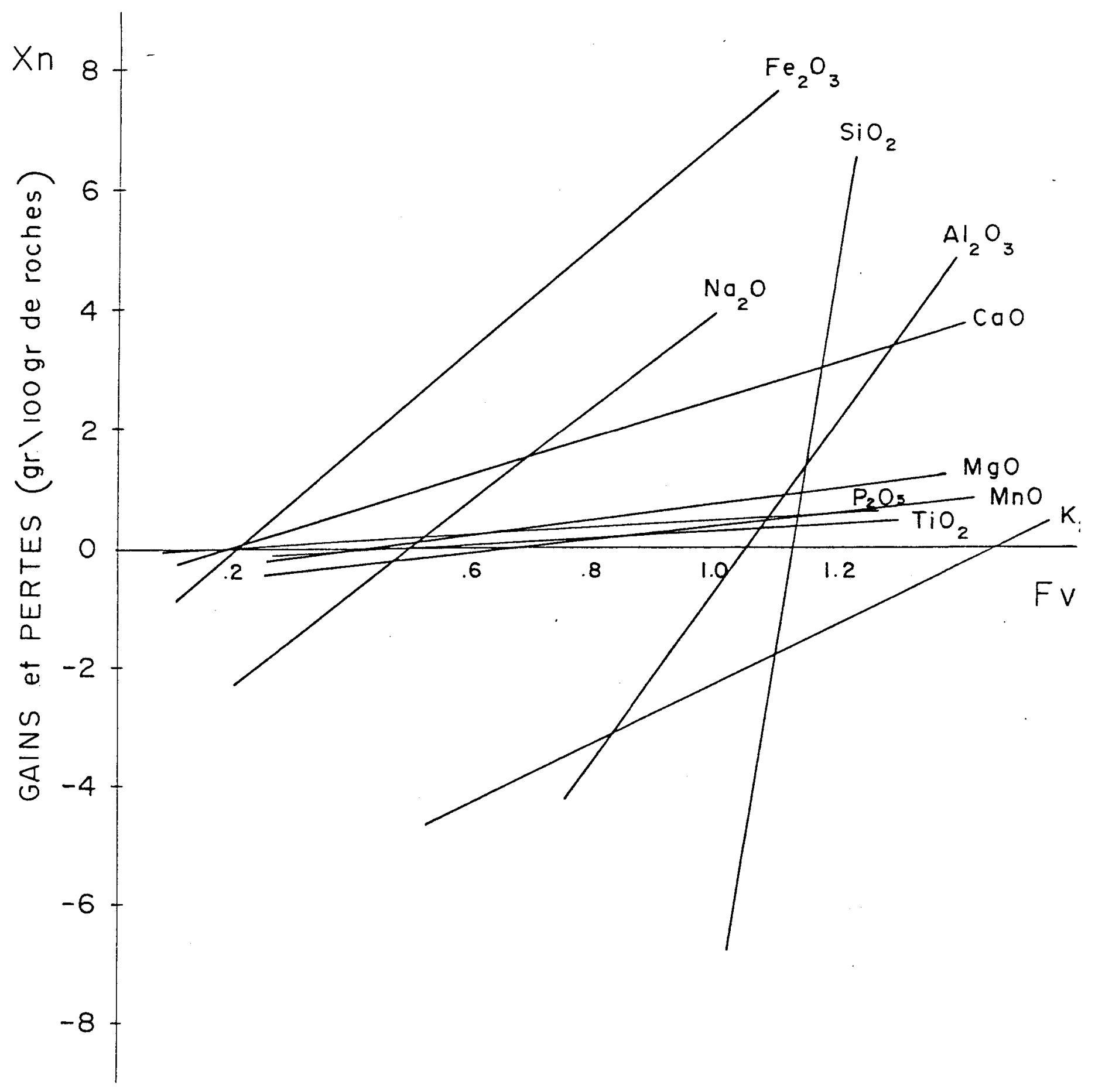

DETERMINATION de FV

Fig.:14 A 


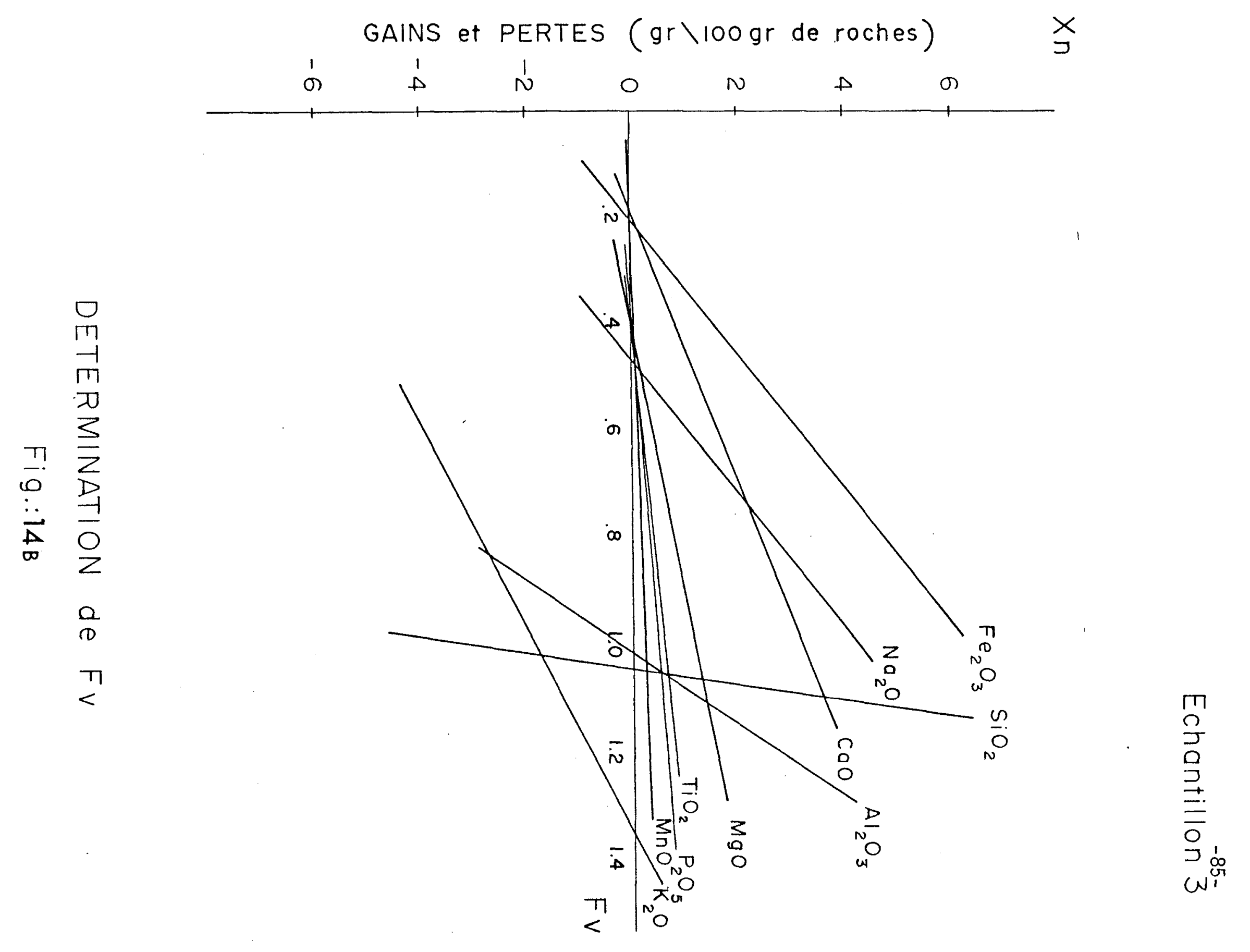


Echantillo ${ }^{-86} \overline{4}$

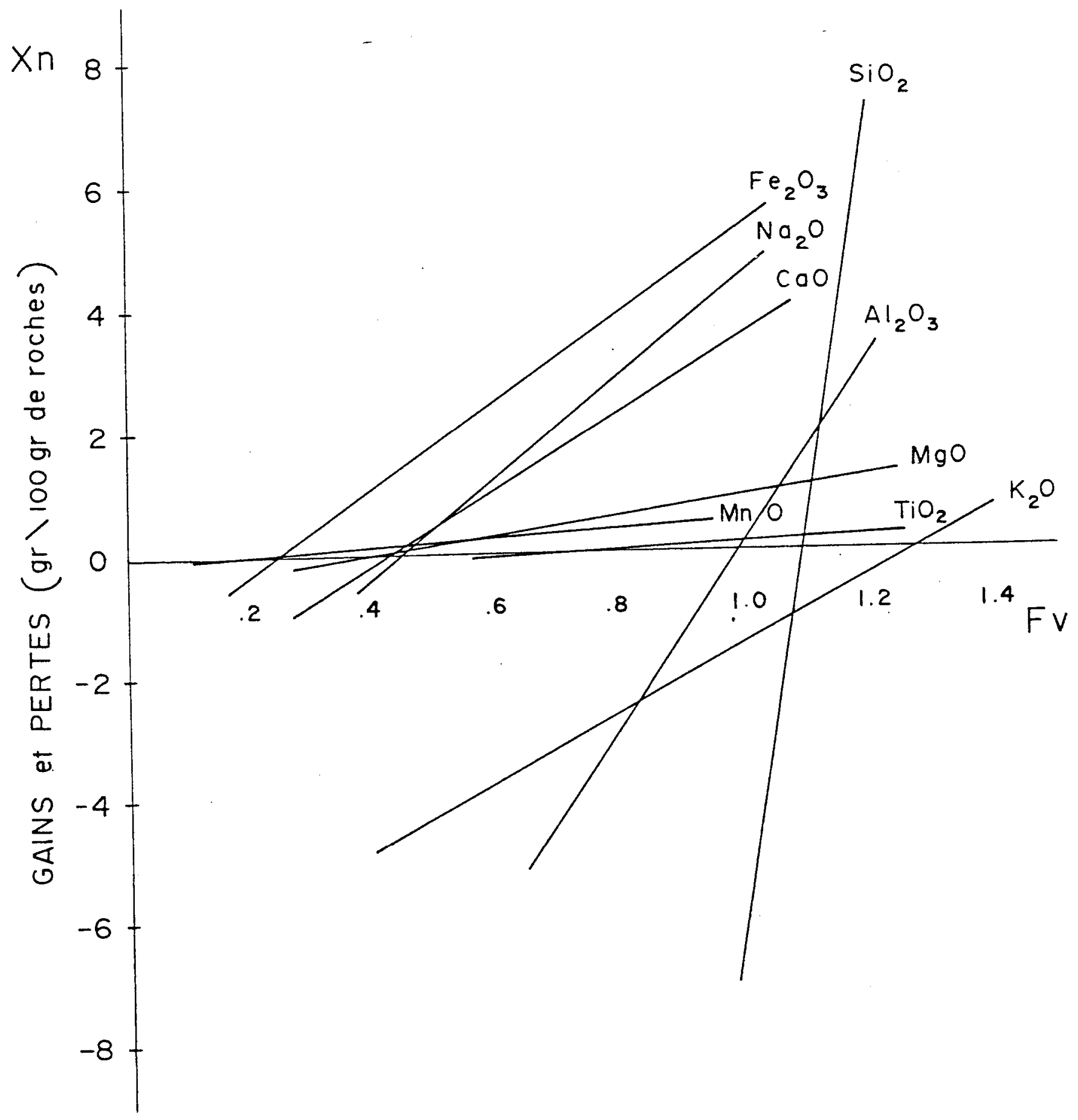

DETERMINATION de FV

Fig.:15A 


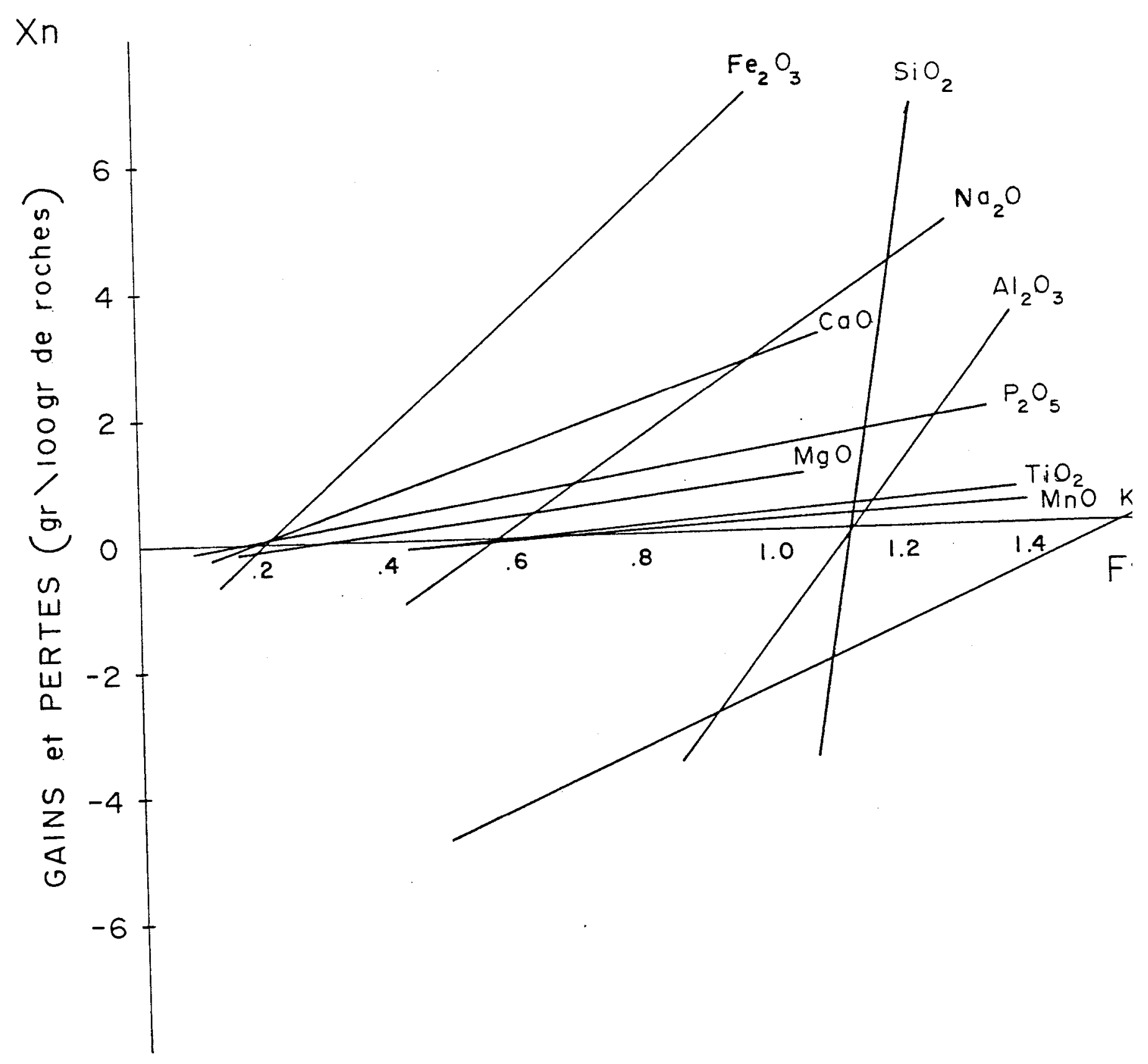

DETERMINATION de FV

Fig.:15B 


\section{Echantillion 6}

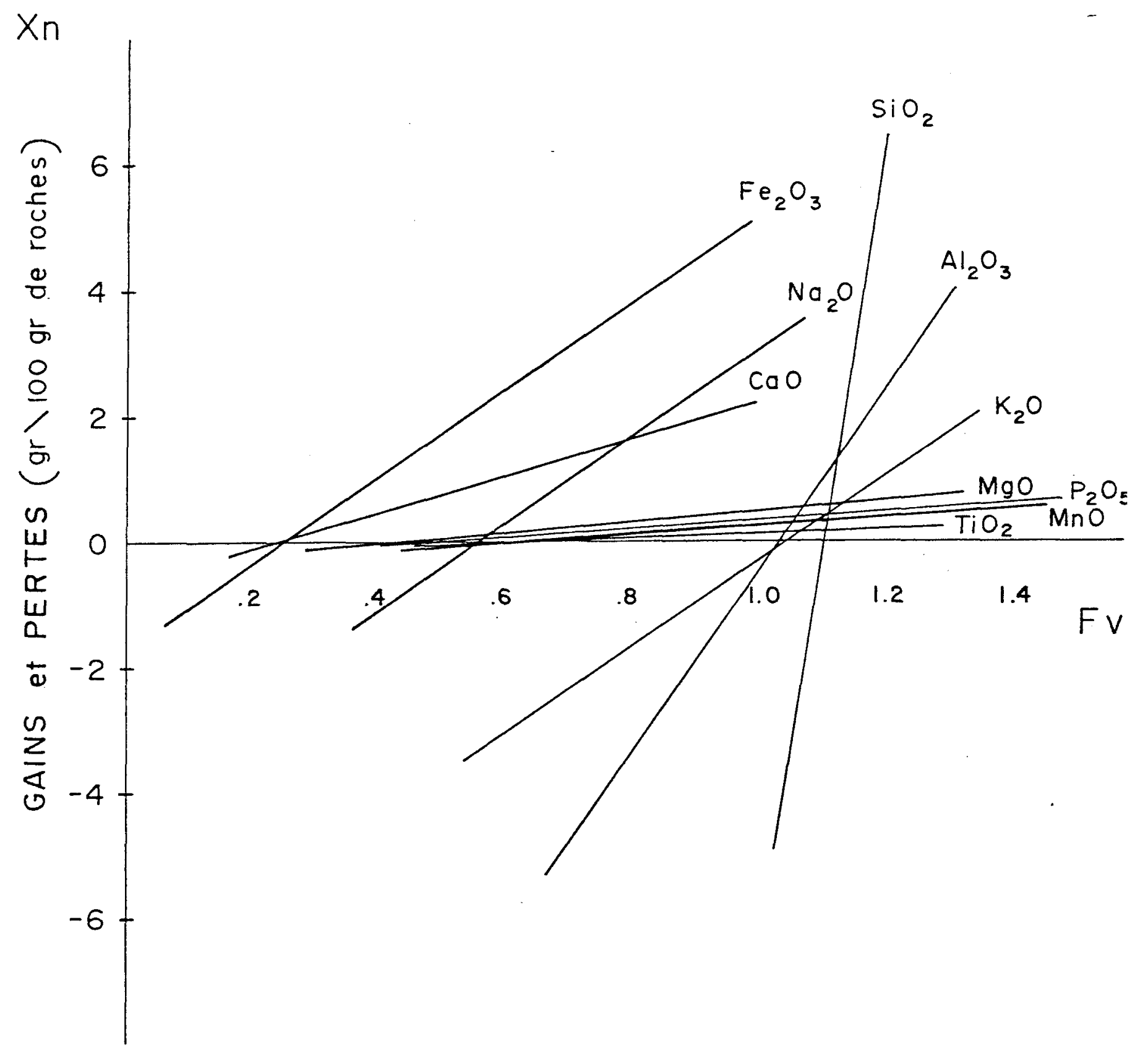

DETERMINATION de FV

Fig.:16A 


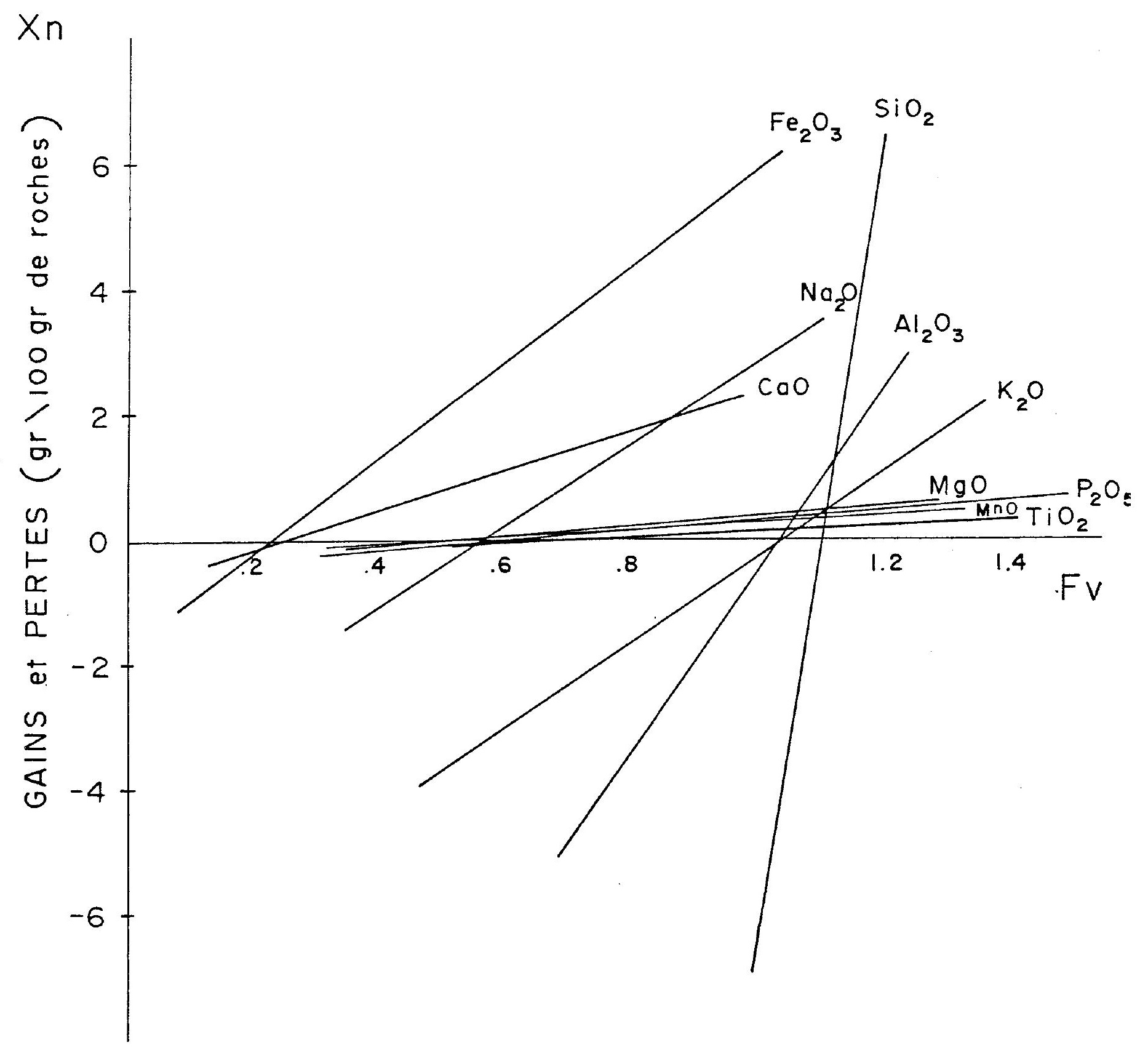

DETERMINATION de FV

Fig.:16B 


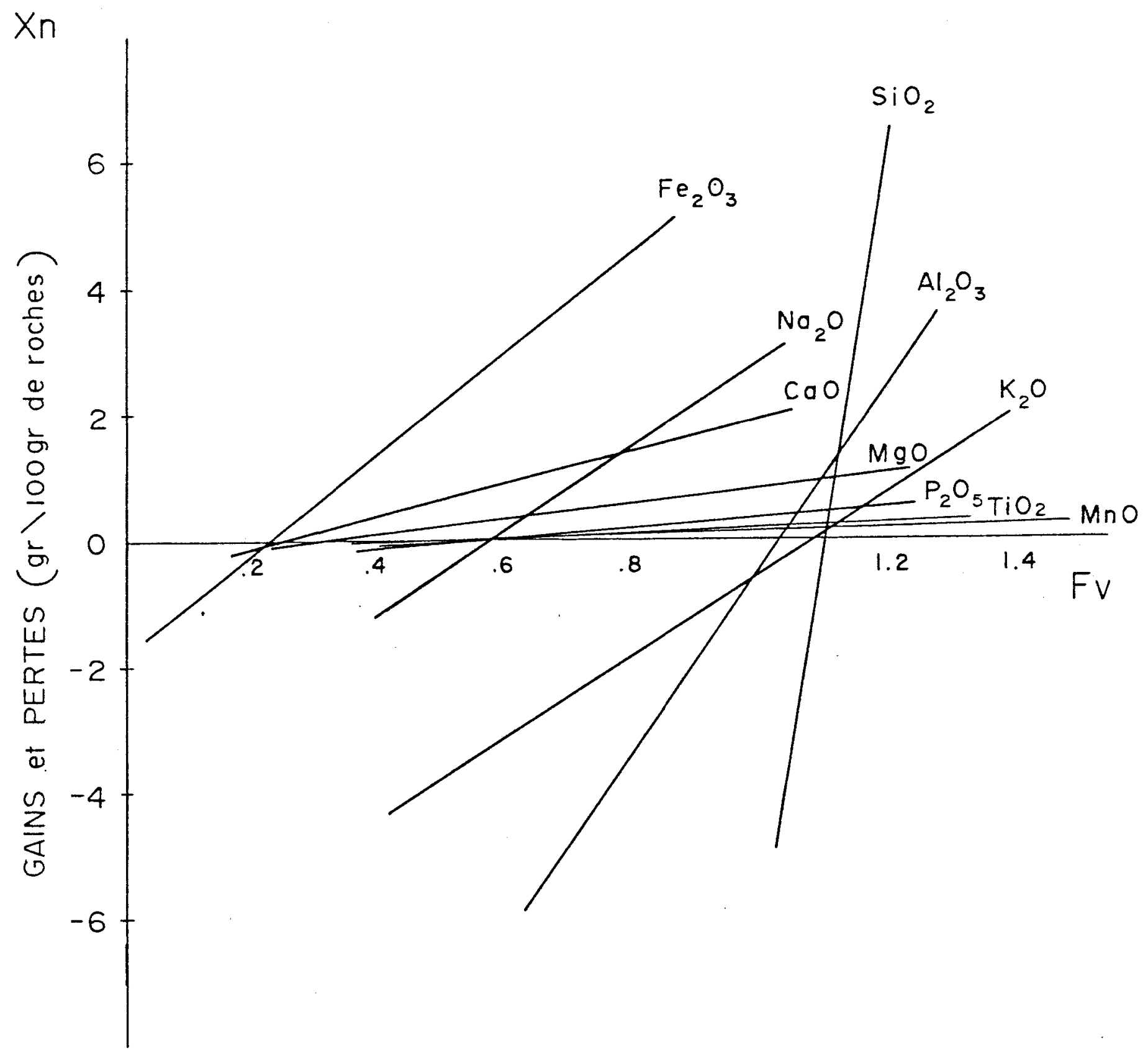

DETERMINATION de FV

Fig.:17A 


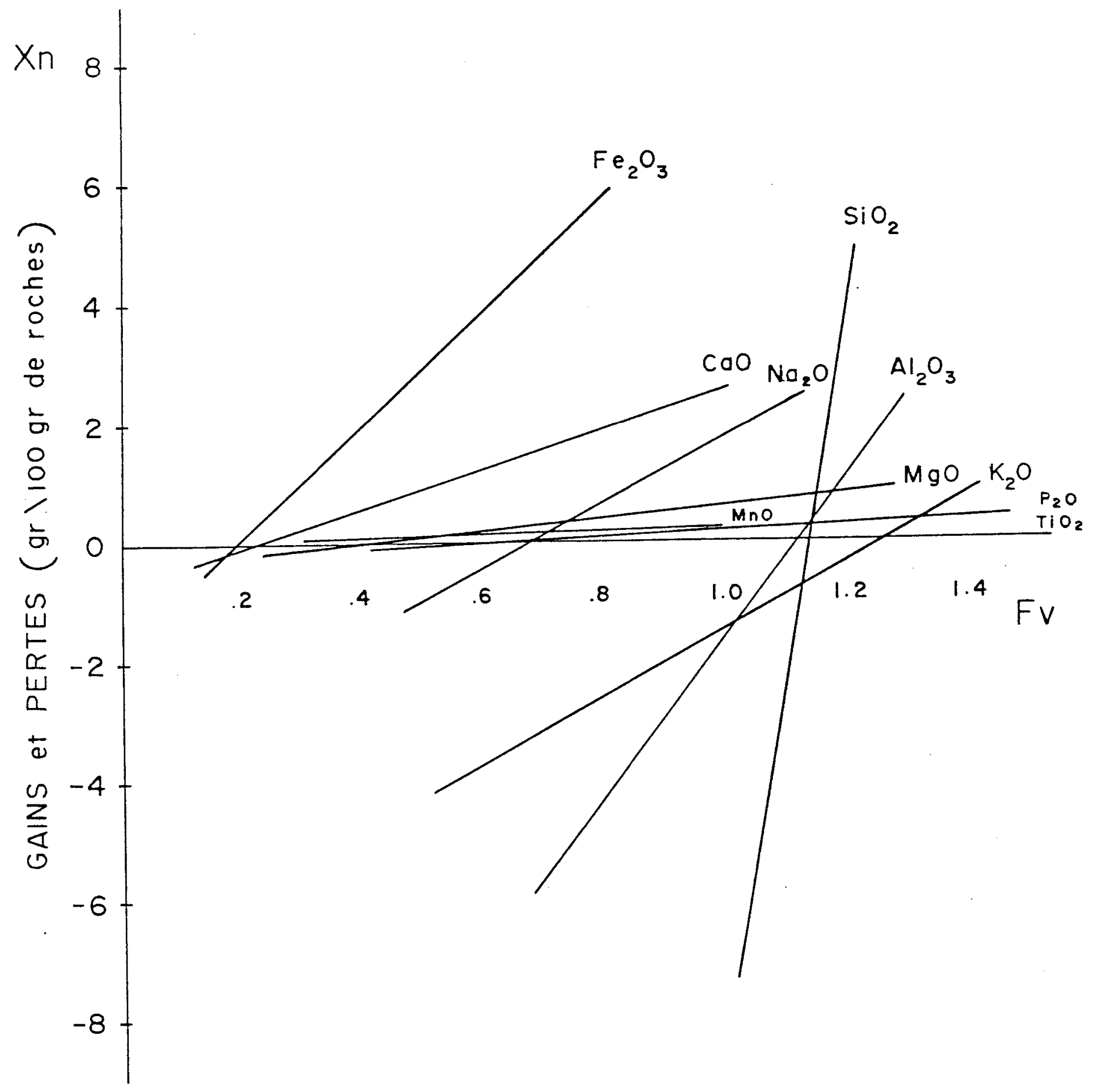

DETERMINATION de FV

Fig.:17B 


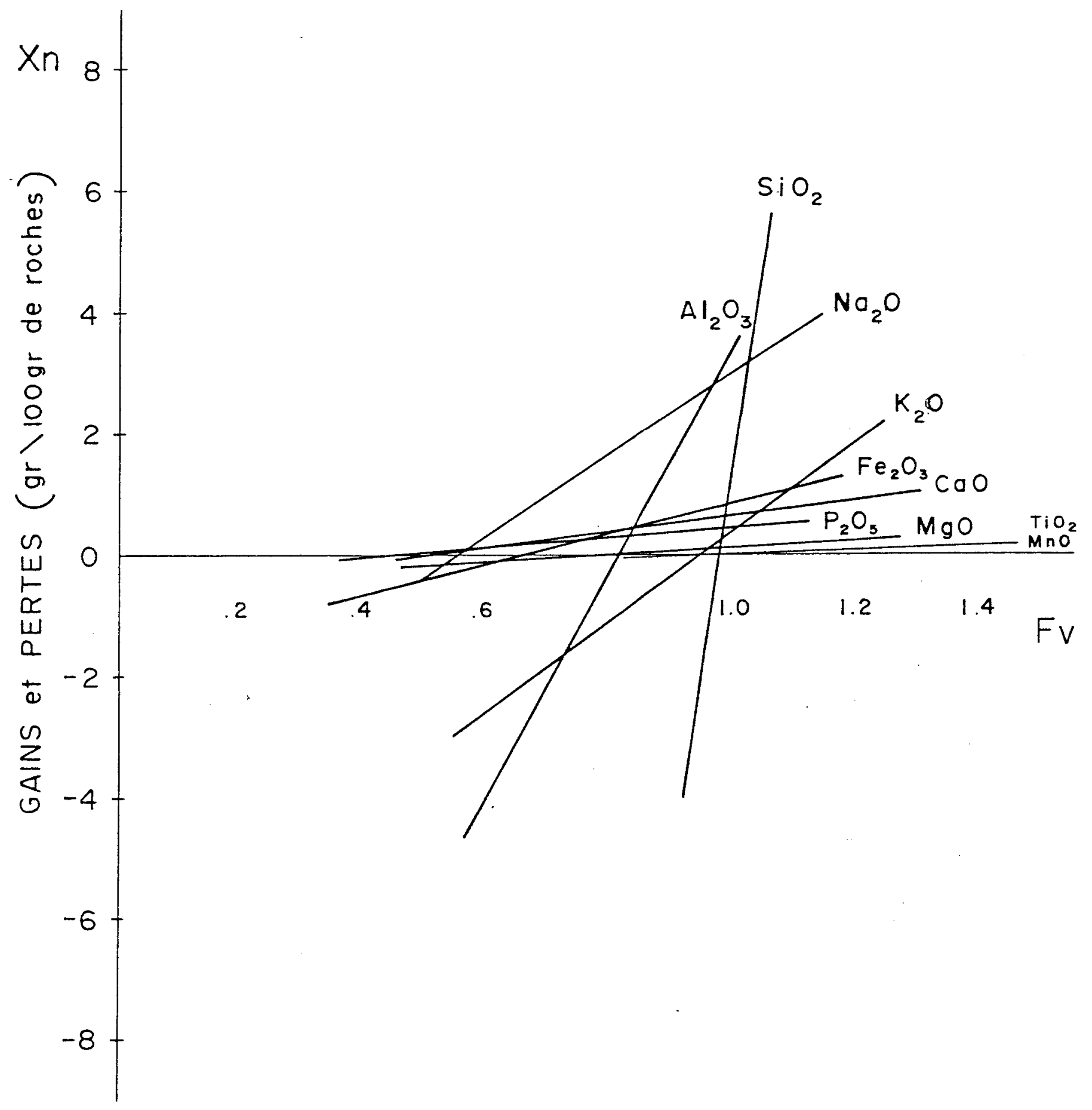

DETERMINATION de FV

Fig.:18A 


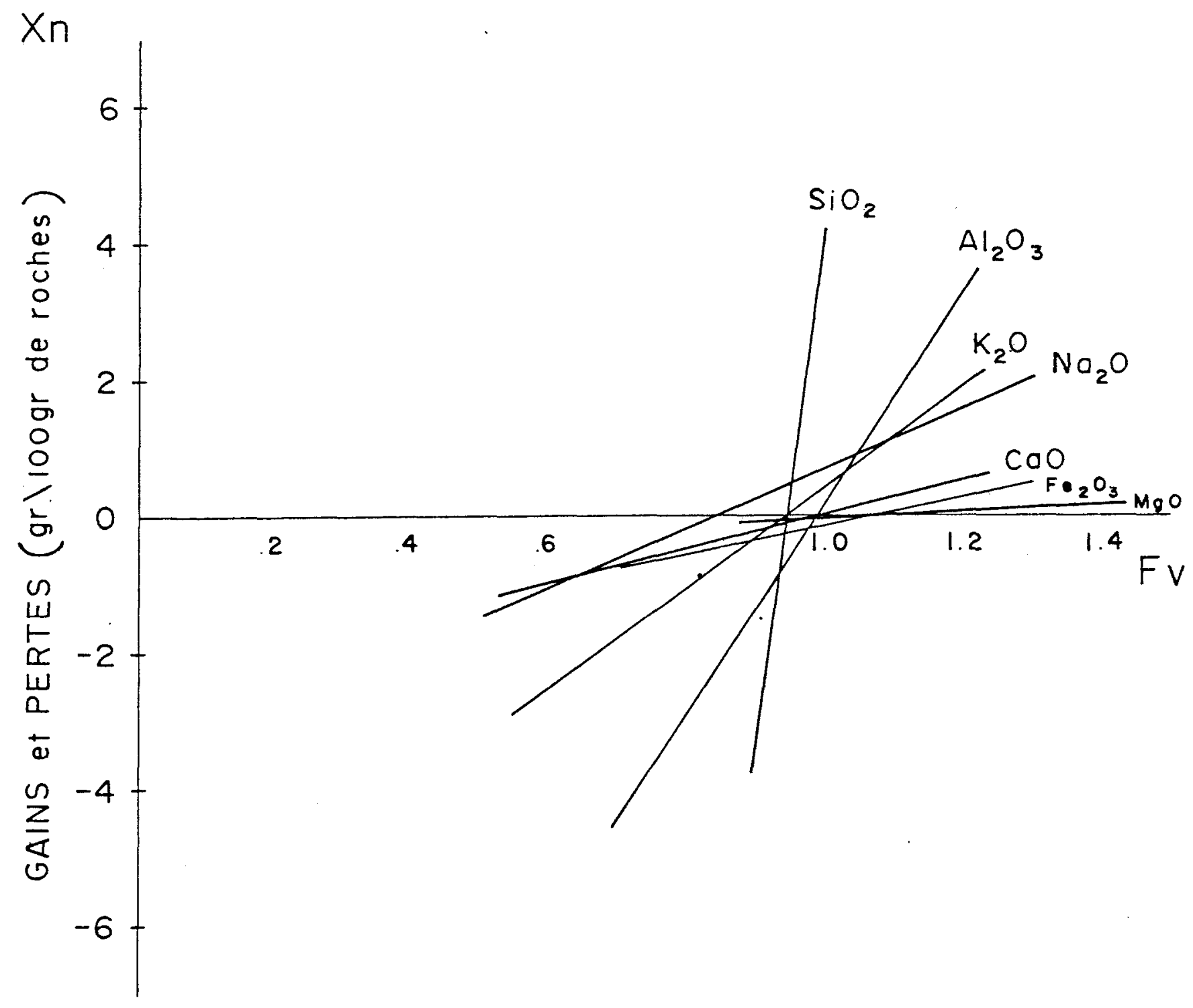

DETERMINATION de FV

Fig.:18B 
Les pentes des éléments $T i, M n, P$ et Mg sont très faibles, ce qui a pour effet de faire varier énormément la valeur de $F_{v}$ à $x_{n}=0$. La nature des pentes est due à une grande variation de \% poids en fonction de la faible teneur de ces éléments dans la roche. Il semble donc erroné d'utiliser des éléments qui sont à l'état de traces pour une détermination exacte de $F_{V}$.

\section{b) - Evaluation des gains et pertes}

La valeur de $F_{V}$ étant déterminée, il est possible à partir de l'équation de base de déterminer les gains et les pertes de chaque échantilion, pour les éléments majeurs et les élëments en trace. Ces valeurs sont compilées dans le tableau 6 .

c) - Diagramme des gains et pertes en fonction de la postion des échantillo!

A partir des données tableau 6 , nous avons construit deux diagrammes des gains et des pertes en fonction de la position de chaque échantilion sur la coupe effectuée sur le terrain. (figure 19) Les diagrammes nous permettent de visualiser les effets du métasomatisme sur les roches de 1'encaissant. Nous constatons que les gains et pertes de matière pour les oxydes $\mathrm{SiO}_{2}, \mathrm{MnO}_{2} \mathrm{Al}_{2} \mathrm{O}_{3}, \mathrm{~K}_{2} \mathrm{O}, \mathrm{Na}_{2} \mathrm{O}$, CaO et $\mathrm{Fe}_{2}$ $\mathrm{O}_{3}$ se répartissent dans des intervalles constants sur toute la coupe; ils tendent vers zéro simultanément et brusquement. Cela implique que le métasomatisme n'est pas progressif, mais constitue un front qui est constant sur une distance de $3000 \mathrm{~m}$ et s'estompe très rapidement. Les autres oxydes, soit $\mathrm{MgO}, \mathrm{P}_{2} \mathrm{O}_{5}$ et $\mathrm{TiO}_{2}$, varient énormément le long de la coupe; ils ne montrent pas le comportement quasi linéaire des oxydes 
TABLEAU 6

VALEURS DE $x_{n}$ POUR LES DIFFERENTS OXIDES DE TOUS LES ECHANTILLONS $\left(F_{V}=1.05\right)$

$\begin{array}{lccccccccccr} & \text { I } & 2 & 3 & 4 & 5 & 6 & 7 & 8 & 9 & \text { IO } & \text { II } \\ \text { Si } & -3.63 & -5.15 & -2.33 & -2.80 & -4.59 & -3.43 & -3.40 & -3.25 & - \text { I.58 } & 2.83 & 5 . I I \\ \text { A1 } & 0.29 & -0.50 & 0.02 & 0.94 & - \text { I.0I } & 0.72 & 0.13 & 0.27 & -0.97 & \text { I.44 } & 0.35 \\ \text { Fe } & 7.23 & 7.12 & 6.47 & 5.66 & 7.6 I & 5.54 & 6.25 & 6.45 & 4.40 & 0.8 I & 0.44 \\ \text { Mg } & 0.73 & 0.70 & \text { I.08 } & 0.8 I & 0.9 I & 0.48 & 0.37 & 0.84 & 0.64 & -0.06 & -0.08\end{array}$

$\begin{array}{llllllllllll}\text { Ca } & 2.56 & 2.64 & 3.19 & 2.39 & 3.02 & 2.39 & 2.4 I & 2.06 & 2.18 & 0.59 & -0.09\end{array}$

$\begin{array}{llllllllllll}\mathrm{Na} & 4.5 \mathrm{I} & 4.65 & 4.30 & 4.99 & 4.05 & 3.37 & 3.18 & 3.13 & 2.05 & \text { I.I4 } & 0.78\end{array}$

$\begin{array}{llllllllllll}K & -2.06 & -2.05 & -I .82 & -I .23 & -2.30 & -0.88 & -0.03 & -0.24 & -0.26 & 0.39 & 0.59\end{array}$

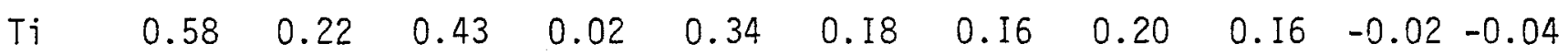

$\begin{array}{llllllllllll}P & 0.4 I & 0.46 & 0.44 & 0.5 I & I .38 & 0.35 & 0.42 & 0.4 I & 0.25 & 0.32 & 0.02\end{array}$

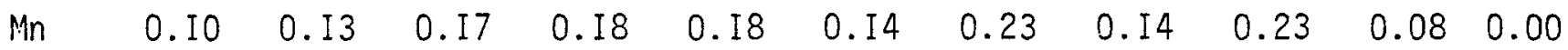

$\begin{array}{llllllllllll}\mathrm{Rb} & -\mathrm{III} & -\mathrm{I} 20 & -\mathrm{I} 07 & -\mathrm{IIO} & -6 \mathrm{I} & -59 & -37 & -90 & -36 & -59 & -25\end{array}$

$\begin{array}{llllllllllll}\text { Sr } & 235 & \text { I } 48 & \text { I } 48 & 895 & -22 & -68 & 33 & \text { I22 } & 350 & 334 & -20\end{array}$

$\begin{array}{llllllllllll}\mathrm{Nb} & \mathrm{I} 47 & 70 & 70 & \mathrm{I} 54 & 7 \mathrm{I} & 58 & \text { I02 } & 87 & 9 & 224 & \text { I35 }\end{array}$

$\begin{array}{llllllllllll}\mathrm{Ba} & -717 & -980 & -785 & -87 \mathrm{I} & -13 \mathrm{I} 8 & -993 & -900 & -865 & -1067 & -754 & -237\end{array}$ 


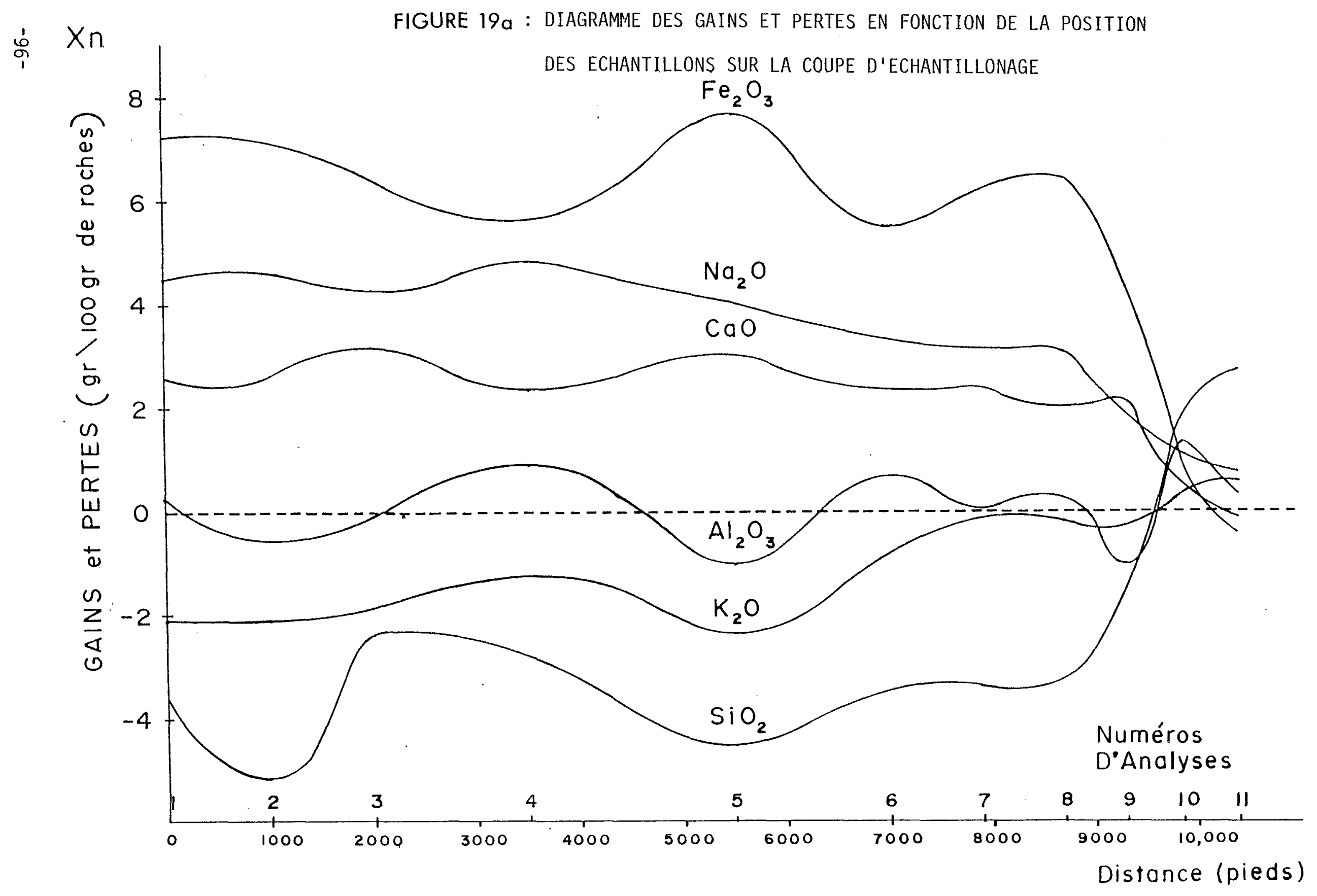




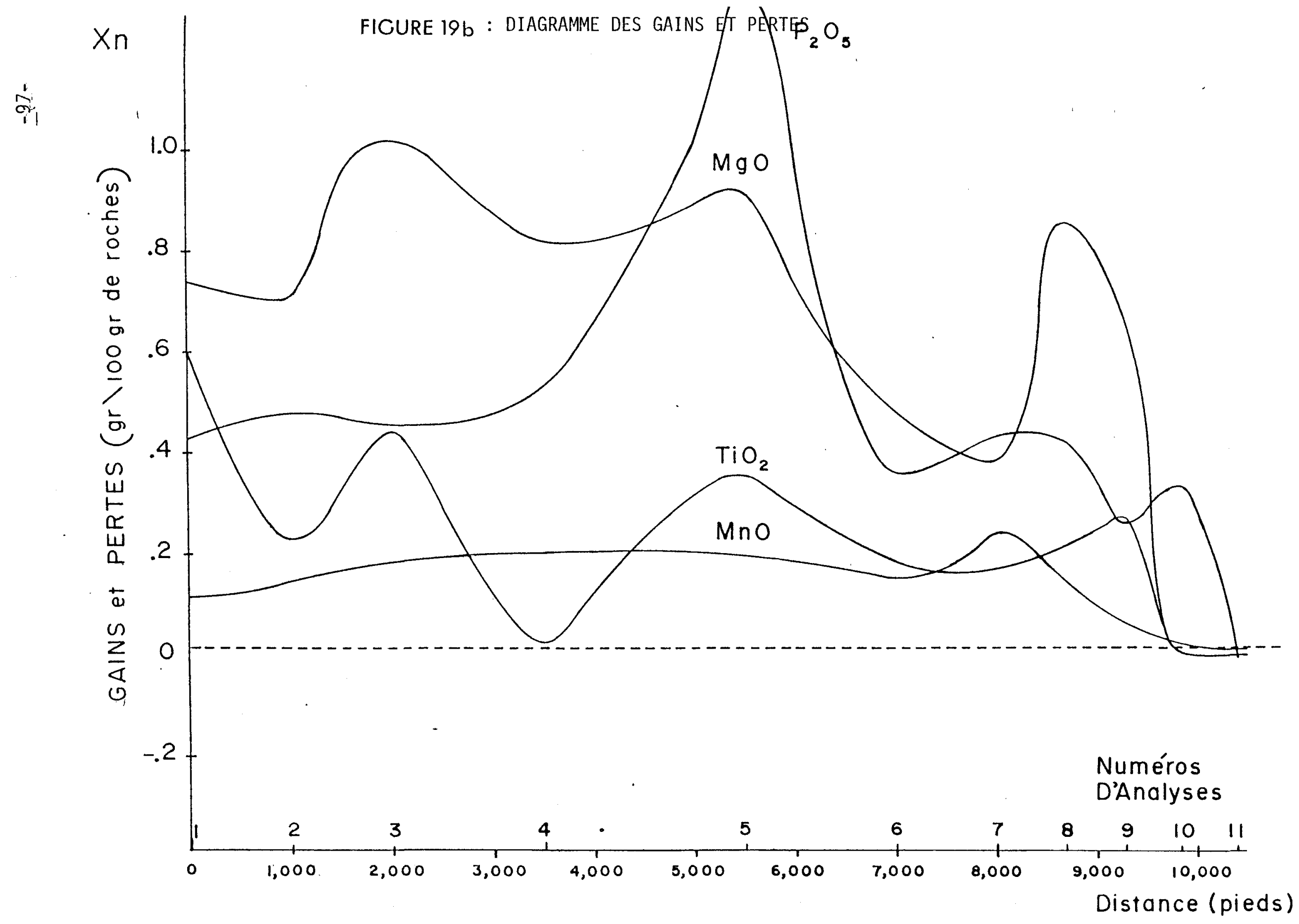


cités précédemment. Il est probable que ces èléments ont été amenēs suite à la mise en place de la carbonatite. Nous les avons observé, associé avec cette dernière, sous forme de l'ilménite, aénigmatite et d'apatite. La mise en place de la carbonatite est un événement nettement postérieur à la fénitisation (métasomatisme I et II). L'encaissant aurait donc subi une fénitisation tardive (métasomatisme IV), en plus de celles identifiées prēcēdemment.

Les èléments qui ont été ajoutēs au système sont: $\mathrm{Fe}_{2} \mathrm{O}_{3}(6.45 \%$ poids), $\mathrm{Na}_{2} \mathrm{O}(4.30 \%), \mathrm{CaO}(2.6 \%), \operatorname{MnO}(0.75 \%)$ et en quantité variable: $\mathrm{P}_{2} \mathrm{O}_{5}, \mathrm{MgO}, \mathrm{TiO}_{2}, \mathrm{Nb}$ et $\mathrm{Sr}$. L'aluminium est le seul élément qui soit relativement stable $(0.10 \%$ poids), ce qui est compatible avec les conclusions des travaux de Gresens (1967). Les éléments qui ont été rejetés du système sont: $\mathrm{SiO}_{2}(3.55 \%), \mathrm{K}_{2} \mathrm{O}(2.10 \%)$, $\mathrm{Rb}$ et $\mathrm{Ba}$. Ces résultats confirment ceux obtenus lors de l'évaluation qualitative. Notons que l'état d'oxidation du fer passe de $\mathrm{Fe}^{+}{ }^{+}$dans les roches non-métasomatisées a $\mathrm{Fe}^{+}+{ }^{+}$ dans les roches métasomatisées.

En comparant nos résultats avec ceux de Appleyard et Woolley (1978), sur les fénites de Skoli et ceux de Siemiatkowska et Martin (1975), sur les quartzites métasomatisées de Mississagi Ont.; nous constatons que les effets du métasomatisme sont les mêmes que ceux calculés dans les fénites de Crevier. Nous observons les mêmes éléments ajoutés et retranchés du système, le changement d'état d'oxidation du fer ainsi que le même type d'assemblage minéralogique, principalement l'aégyrine. 


\subsection{Evaluation du métasomatisme}

L'étude pétrographique et pétrochimique des roches de T'encaissant métasomatisé du complexe, nous permet maintenant de faire la synthèse des phénomènes qui ont affecté ces roches. Le modèle proposé (figure 20) est fait d'une roche parentale composée de quartz, de plagioclases (An 30), de feldspaths alcalins perthitiques, de biotite et de sphène. Le produit des réactions métasomatiques est composé d'aégyrine, d'arfvedsonite, de plagioclases (An 30), de feldspaths alcalins perthitiques, de sphène, de zircon, d'aénigmatite et de calcite. Accompagnant les changements de l'assemblage minéralogique original, nous avons les changements texturaux suivants: le remplacement du quartz, de la biotite et des feldspaths alcalins (à granulométrie fine) par l'aégyrine, le remplacement de l'aégyrine par l'arfvedsonite (remplacement local), augmentation graduelle et substantielle du degré de perthitisation dans les gros cristaux de feldspaths alcalins ainsi que l'apparition d'une phase antiperthitique dans les plagioclases. Bien qu'aucune texture de remplacement de la biotite n'est observée, nous croyons que cette dernière ait contribuée à la formation de l'aégyrine. La morphologie des bandes d'aégyrine dans les gneiss oeillés et les gneiss quartzo-feldspathiques à biotite et hornblende, est similaire aux bandes de biotite et de biotitehornblende de ces mêmes roches. Borodin et Pavleko (1971) citent des réactions identiques dans des roches métasomatisées de composition similaire aux pegmatites de l'encaissant du complexe.

Le métasomatisme I et II ont produit l'aégyrine, par la réaction avec les minéraux cités précédemment, ainsi que l'apport de fer et de sodium. Le remplacement de l'aégyrine par l'arfvedsonite pourrait être expliqué par la réaction de l'aégyrine avec de la vapeur d'eau sous des 
conditions favorables, à l'extrémité du front de fénitisation. L'apport de calcium et de strontium n'a pas été identifié à une ou des phases minérales précises. Le métasomatisme IV, associé à la mise en place erratique de la carbonatite, a produit un apport de $\mathrm{Ti}$, $\mathrm{P}$ et $\mathrm{Mg}$ pour former les minéraux cités précédemment, et possiblement du zircon. Ce minéral est commun aux métasédiments et aux pegmatites du Grenville, mais pas en aussi grande quantité ( 1 à $2 \%$ ) que dans les roches de l'encaissant du complexe de Crevier.

Tous les transfers de masse ont produit une augmentation de $5 \%$ du volume initial de roche.

L'auréole de métasomatisme forme une couronne de 2 a $3 \mathrm{~km}$ de largeur autour du complexe, qui montre les même effets que ceux décrits dans la coupe d'échantillonnage utilisée pour cette étude. L'ampleur du phénomène en surface est considérable; il implique un grand apport de volatils, caractéristique de l'état résiduel des magmas aicalins (Borodin, 1971).

L'évaluation du métasomatisme a été faite avec des analyses totales de roches et les résultats sur l'assemblage minéralogique ont été déduits à l'aide de l'étude pétrochimique. Il serait utile de vêrifier nos conclusions au niveau des minéraux, en isolant ces derniers et, à l'aide de leur formute stozchiométrique et de leur analyse, refaire le même processus utilisé pour l'assemblage de la roche. 
FIGURE 20

Roche parentale

100 Unités de volume

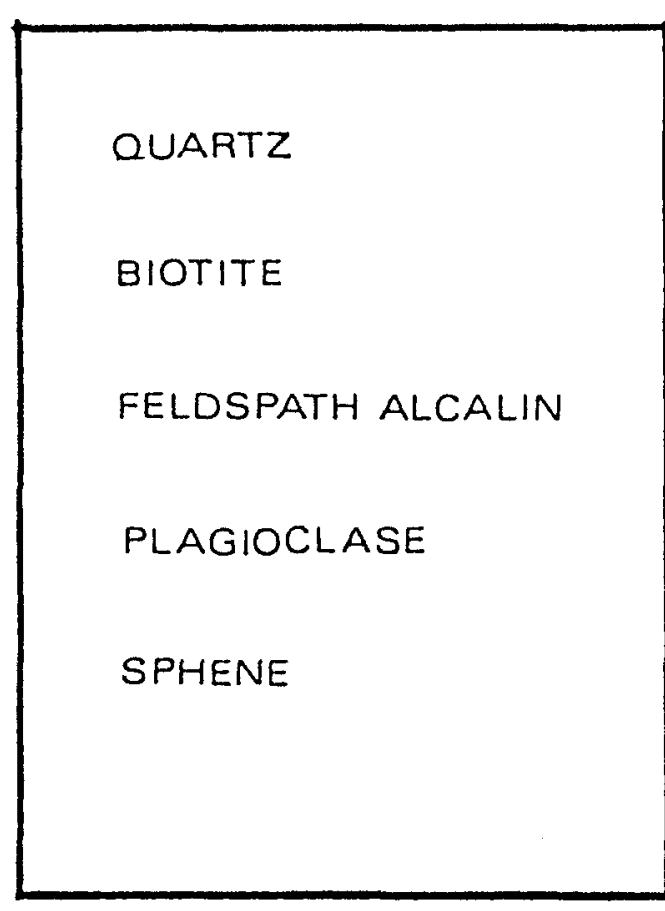

METASOMATISME 1

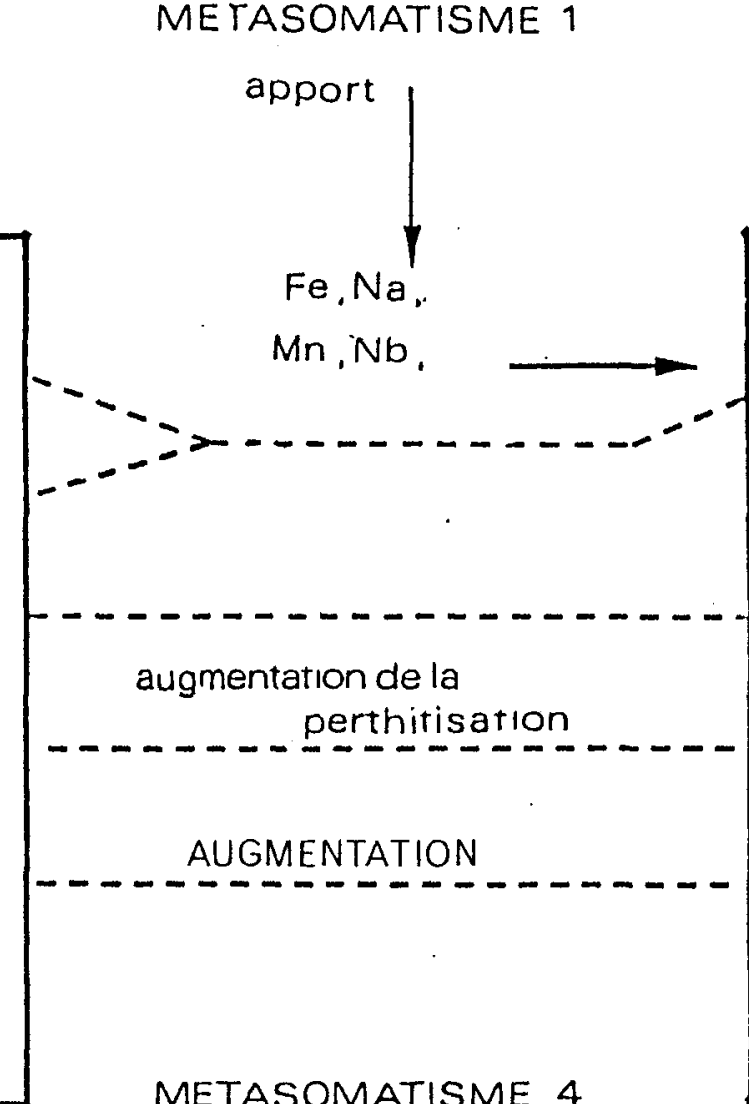

METASOMATISME 4 $\mathrm{Ca}, \mathrm{CO}_{2}, \mathrm{P}, \mathrm{TI}$. $\mathrm{Mg}, \mathrm{Zr}, \mathrm{Sr}$
Roche metasomatisee 105 Unités de volume

\begin{tabular}{|l|}
\hline AEGYRINE \\
ARFERDSONITE \\
FELDSPATH ALCALIN \\
PLAGIOCLASE \\
SPHENE \\
AENIGMATITE
\end{tabular}

CALCITE APATITE ZIRCON ILMENITE 
CONCLUSIONS

Le complexe alcalin de Crevier est composé de sept lithologies, soit: les monzosyénites à nêphêline I, les diorites a biotite, 1a monzosyênite à néphêline II (pegmatitique), la monzosyēnite à néphểine et biotite III, la monzosyēnite à néphēline IV (dykes tardifs), la carbonatite et la monzosyénite. L'ordre dans lequel sont énumérées les Tithologies, correspond à la succession chronologique de ces dernières. La monzosyénite à néphéline I et la diorite à biotite ont une mise en place contemporaine. La position de la monzosyénite dans la colonne stratigraphique n'a pu être établi, vu le manque d'informations.

Deux phases minéralisées importantes sont associées à ces 7ithologies, soit: une phase de minéralisation disséminée de tantaleniobium dans la monzosyēnite à nēphéline pegmatitique II et une deuxième phase d'uranium-niobium associēe à la mise en place de la carbonatite.

Les lithologies ont été groupées dans des unités selon l'abondance relative des types de roche observé sur le terrain et dans les forages.

La minéralogie des roches du complexe est relativement simple (albite, néphēline, feldspaths alcalins et biotite). Les phases mafiques telles que les amphiboles et pyroxènes, sont absent de l'assemblage minêralogique. 
L'étude de l'évolution géochimique des roches du complexe fait ressortir qu'elles ont subi une faible différenciation. Leur comportement est similaire à celui décrit par Bowen (1958), le "petrogeny's residua system" où le magma, en se refroidissant, s'enrichit en ses propres éléments. Nous avons cru pouvoir évaluer la pression à laquelle le massif s'est mis en place. Nos estimations sont de l'ordre de 7 à $8 \mathrm{~kb}$ (une profondeur de $40 \mathrm{~km}$ ), ce qui représente le niveau d'érosion probable du site du complexe.

L'étude géochimique a aussi fait ressortir que le comportement des diorites à biotite diffère des autres roches du complexe. Nous croyons que ce faciès représente des restites de la roche encaissante, partiellement assimilée lors de la mise en place de la monzosyénite à néphêtine I.

L'étude sur le terrain des relations du contact plutonencaissant, a montré le caractère nettement intrusif du massif alcalin à travers les gneiss qui composent l'encaissant.

Nous avons proposé un modèle de contrôle structural qui a permis la mise en place du massif. Ce modèle est relié aux structures secondaires qui se rattachent à de grandes structures marquées par les rivières du nord du Lac Saint-Jean. Il en est de même pour les structures de l'unité 1 du complexe. Le fort rubannement qui la caractérise pourrait être le résultat du débitage de la roche lors du relâchement d'efforts de compression. Cette hypothèse est compatible avec le modèle structural citê précédemment, ainsi que l'hypothèse proposée pour expliquer l'origine de la diorite à biotite. 
L'effet majeur de l'intrusion du massif est la création d'une auréole de métasomatisme dans les roches de l'encaissant. Le métasomatisme est le premier événement, dans la succession chronologique de ces derniers, inhērent au complexe alcalin de Crevier. La fénitisation a produit principalement l'aégyrinisation des roches de l'encaissant. Nous avons évalué les effets du métasomatisme sur ces roches à l'aide d'études pétrochimiques et géochimiques. Elles ont permi d'identifier et de quantifier les changements de l'assemblage minéralogique original de la roche, soit la formation d'aégyrine aux dépends du quartz, de la biotite et des feldspaths alcalins par l'apport de fer, de sodium, de calcium en plus de l'apport de manganèse, de strontium et de niobium. Ces apports sont accompagnés de la désilicification des roches de l'encaissant ainsi qu'une perte de potassium, de rubidium et de barium. Une deuxième vague de fénitisation a été identifiée. Celle-ci est reliée à la mise en place de la carbonatite. Elle a produit, dans les roches de l'encaissant, la formation de calcite, d'apatite, d'ilménite et d'aénigmatite par l'apport de $\mathrm{CO}_{2}$, de phosphore, de titane et de magnésium.

L'étude des roches de l'encaissant nous a aussi permis de situer l'intrusion du massif alcalin, par rapport aux événements grenvillien L'aégyrinisation des pegmatites post-orogénique est l'évidence que le complexe alcalin s'est mis en place après l'orogénie grêvilienne.

Un grand nombre d'auteurs relient ce genre d'intrusions aux systèmes de rifts continentaux . Ce type de structure donne accès à la croute supérieure les magmas du manteau et de la croute inférieure. La 
proximitê du grabben du Saguenay est un argument en faveur de la relation du complexe de Crevier avec ce dernier. Nous en sommes qu'aux présomptions car aucune donnée géochronologique n'est disponible pour le moment et les études sur le graben du Saguenay sont embryonnaires. Nous avons la conviction que les travaux que nous avons effectué sur le complexe igné alcalin de crevier, ont grandement contribué à l'amélioration de son image gêologique. Mais de nombreuses questions sont restées en suspens. Ne mentionnons que l'origine de la carbonatite (magmatique ou hydrothermale), l'origine des monzosyénites à néphéline et des monzosyénites (fusion partielle d'un matériel approprié au niveau de la croute inférieure ou cristallisation fractionnēe au niveau du manteau?); des études sur les inclusions fluides et les isotopes du rubidiumstrontium pourraient répondre à ces questions. Mentionnons aussi que certains sujets traités dans le cadre de cette étude devront faire l'objet de travaux plus poussês; des études sur la structure de l'encaissant du complexe seraient nécessaires pour confirmer ou infirmer le modèle structural que nous avons proposé. Les études quantitatives sur les effets du métasomatisme, devront être étayées par des travaux plus poussés à l'aide notamment de la micro-sonde, ainsi que des travaux devront être entrepris sur la composition des phases volatiles qui ont provoquées le métasomatisme. 


\section{REMERCIEMENTS}

L'auteur tient à remercier toutes les personnes qui ont contribué, de près ou de loin à la production de ce mémoire.

J'exprime ma reconnaissance à Monsieur Gérard Woussen qui n'a cessé de me prodiguer suggestions, conseils et encouragements, ainsi que Messieurs E.H. Chown et Guy Perrault pour les conseils et les critiques pertinents qu'ils m'ont prodigués.

Je suis très reconnaissant envers Messieurs Fernand Dubuc et Jacques Bonneau, de la Soquem, qui ont fourni le support logistique et financier des travaux de terrain et d'analyse.

Je tiens également à remercier les techniciens et les dessinateurs de L'U.Q.A.C. et de la Soquem.

Je veux souligner la contribution morale de mon épouse Sylvie, tout au long de mes travaux. 


\section{ANNEXE I}

Méthode d'échantillonnage

Des analyses d'êléments majeurs et éléments en traces, d'une série de roches de l'encaissant métasomatisé et non métasomatisé, ont été demandées, dans le but de faire une évaluation géochimique du métasomatisme. Vu le caractère très hétérogène des différents faciès observés dans l'auréole de métasomatisme, il a été décidé de faire l'évaluation géochimique à partir des pegmatites. Ce choix est du surtout a l'homogénēité de ces roches et leur grande occurrence dans cette zone. La collecte des échantillons s'est faite à l'aide de tirs à la dynamite. Le poids de chaque échantilion receuilli est d'environs $18 \mathrm{~kg}$ de roche frâche. La forte granulométrie des pegmatites justifie ce poids, pour la qualité de représentativité des analyses. 


\section{ANNEXE II}

\section{Méthodes d'analyses}

Toutes les analyses chimiques utilisées dans cette étude, ont été effectuées par le ministēre des Richesses Naturelles (Centre de Recherches Minérales) et subventionnées par la Sociēté Québécoise d'Exploration Miniēre (SOQUEM).

Les méthodes d'analyses sont les suivantes:

- Fluorescence $X$, pour les éléments: $\mathrm{Al}_{2} \mathrm{O}_{3}, \mathrm{MgO}, \mathrm{CaO}, \mathrm{Na}_{2} \mathrm{O}, \mathrm{SiO}_{2}$ $\mathrm{K}_{2} \mathrm{O}, \mathrm{TiO}_{2}, \mathrm{MnO}, \mathrm{CO}_{2}$ $U$, Th, Rb, $\mathrm{Sr}, \mathrm{Ba}, \mathrm{Nb}$

Ta.

- Gravimétrie, pour la détermination de $\mathrm{H}_{2} \mathrm{O} \mathrm{t}$.

- Colorimétrie, pour la détermination de $\mathrm{P}_{2} \mathrm{O}_{5}$

- Absorption Atomique, pour la détermination de $\mathrm{Fe}_{2} \mathrm{O}_{3}$

- Volumétrié, pour la détermination de FeO 


\section{ANNEXE III}

Schéma de broyage.
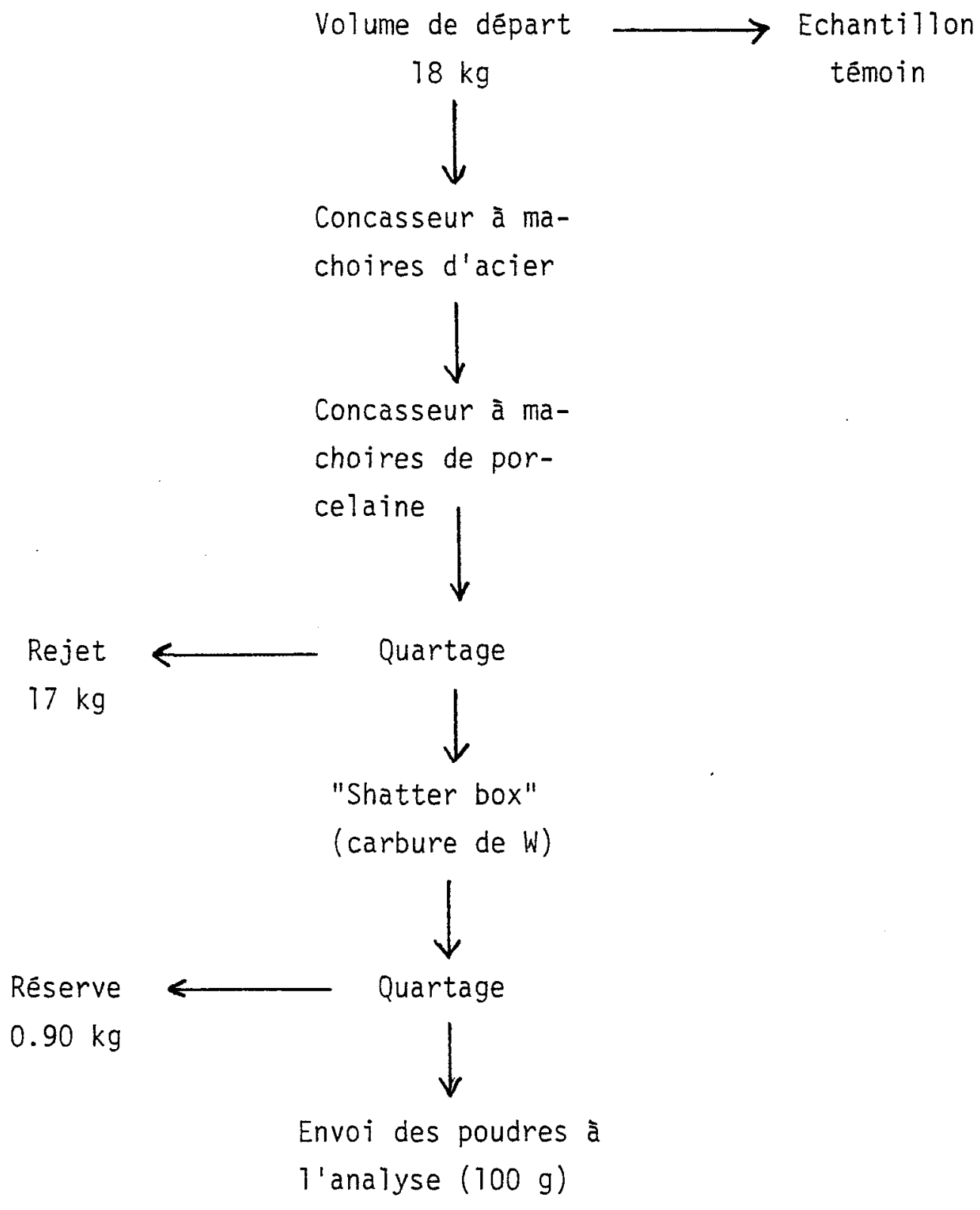


\section{ANNEXE IV}

Méthode de cạlcul de la de_densit té

La densité des pegmatites a èté déterminée par la mesure du poids des échantilions immergés dans le méthanol et par la suite la détermination du volume déplacé.

Les étapes du calcul sont les suivantes:

1- Découpage de chaque échantilion $(1 \mathrm{~kg})$ en plaquettes de $3 \times 5 \mathrm{~cm}$. (5 plaquettes par échantition)

2- Mesure du poids de chaque plaquette $\left(P_{p}\right)$

3- Mesure du poids de chaque plaquette immergées dans le méthanol $\left(P_{j}\right)$; durée d'immersion: 3 minutes

4- Calcul de la densité de chaque plaquette à l'aide des équations suivantes:

$$
\begin{aligned}
& \text { 1) }-v_{d}=\frac{p_{p}-P_{i}}{d_{m}} \\
& \text { 2) } d_{R}=\frac{P_{p}}{\frac{V_{d}}{d^{\prime}}}
\end{aligned}
$$

$V_{d}$ : Volume déplacé

$\mathrm{d}_{\mathrm{m}}$ : Densité du méthanọ, 0.7914 à $20^{\circ} \mathrm{C}$

$d_{R}$ : Densité de la roche

5- Calcul de la densité de la roche en calculant la densité moyenne des plaquettes. 
Deux sources principales d'erreur peuvent influencer les résultats des calculs: perte de matériel entre la pesée à sec et la pesée immergée. Calcul erroné du volume déplacé du au fissures et cavités de la roche, le liquide peut ne pas s'introduire dans ces fissures et cavitês.

La première source d'erreur peut-être contrôlée que par des soins particuliers lors de la manipulation des échantilions et ne peutêtre évaluẽe. Par contre dans le cas de la deuxième source d'erreur un test a été effectué. Nous avons laissé tremper une plaquette pendant trois heures dans le méthanol, en prenant soin de mesurer le poids de la plaquette après trois minutes d'immersion. Les différences de poids enregistrées sont de 0.13 grammes, ce qui impute sur le calcul de la densité une erreur de \pm 0.01 . Cette marge d'erreur est qualifiée de négligable, car nos échantillons montrent un intervalle de valeurs de densité assez grand, valeurs variant entre 2.86 et 2.59 . 
Détermination de la calcicité des plagioclases

La calcicité des plagioclases a été déterminée à l'aide d'un microscope équipé d'une platine universelle. La méthode employée est celle proposeee par Roubault (1963) soit (010) vertical et (100) vertical Elle consiste à choisir plusieurs sections propices à la mesure, les amener à l'horizontale de la platine et mesurer leur angle d'extintion. Cette méthode a une justesse de \pm An 2 selon l'auteur, ce qui est jugé suffisant dans le cadre de cette étude. 
Exemple de calcul de l'êvaluation quantitative du métasomatisme.

Analyses traitēes:

- analyse \#14, roche parentale

- analyse \# 2, roche métasomatisée

Le traitement des données s'est fait à l'aide de deux programmes utilisables sur une calculatrice HP-35, de Hewlett-Packard. Ces deux programmes sont insérés à la fin de cette annexe.

Données:

valeurs de $C_{n}$
\#2

Si 0.6089

A] 0.1443

Fe 0.0816

Mg 0.0102

Ca 0.0308

$\mathrm{Na} \quad 0.0799$

K 0.0456

Ti 0.0043

P $\quad 0.0046$

Mn $\quad 0.0015$

Poids spécifique
\#14

0.7106

0.1503

0.0171

0.0040

0.0069

0.0378

0.0699

0.0025

0.0004

0.0003

2.623 
1)- Détermination de $F_{v}$

A l'aide du programme 1 , nous obtenons les valeurs de $x_{n}$ pour $F_{V}=0.6,0.8,1.0,1.2$ et 1.4 (tableau 1$)$

2)- Construction du diagramme $C-V$ à partir des donnēes du tableau 1

3)- Détermination de $x_{n}$

Ces valeurs sont déterminées à l'aide de l'équation de base

et du programme 2 .

Programme 1

\begin{tabular}{|c|c|c|c|}
\hline 00 & & $16-$ & MEMOIRES \\
\hline 01 & . & 17 ENTER & \\
\hline 02 & 6 & 181 & $0 \rightarrow F_{V}(.6)$, incorporé \\
\hline 03 & STO 0 & 190 & au programme \\
\hline 04 & RCL 0 & 200 & $1 \rightarrow c_{n}^{\beta}$ \\
\hline 05 & ENTER & $21 x$ & \\
\hline 06 & $\mathrm{RCL} 1$ & 22 f PAUSE & $2 \rightarrow \rho^{\alpha}$ \\
\hline 07 & $x$ & 23. & $3 \rightarrow \rho^{\beta}$ \\
\hline 08 & ENTER & 242 & \\
\hline 09 & $\mathrm{RCL} 2$ & 25 STO - 0 & $4 \rightarrow c_{n}^{\alpha}$ \\
\hline 10 & $x$ & 26 RCL 0 & \\
\hline 11 & ENTER & 27 RCL 5 & $5 \rightarrow 1.4$ \\
\hline 12 & $\mathrm{RCL} 3$ & $28 x=y$ & \\
\hline 13 & $\div$ & $29 \mathrm{R} / \mathrm{S}$ & \\
\hline 14 & ENTER & 30 GT) 04 & \\
\hline & RCL 4 & & \\
\hline
\end{tabular}


Programme 2

$\begin{array}{lllll}00 & & 14 & 6 & F_{V}=1.05 \\ 01 & 1 & 15 & 2 & \rho^{\alpha}=2.623 \\ 02 & \cdot & 16 & 3 & \\ 03 & 0 & 17 & \div & \\ 04 & 5 & 18 & \text { ENTER } \\ 05 & \text { ENTER } & 19 & \text { CRL } 3 & \\ 06 & \text { RCL 1 } & 20 & - & 1 \rightarrow C_{m}^{\alpha} \\ 07 & \times & 21 & \text { ENTER } & \\ 08 & \text { ENTER } & 22 & 1 & 2 \rightarrow \rho^{\beta} \\ 09 & \text { RCL 2 } & 23 & 0 & 3 \rightarrow c_{m}^{\rho} \\ 10 & x & 24 & 0 & \\ 11 & \text { ENTER } & 25 & x & \\ 12 & 2 & & & \\ 13 & \text {. } & & & \end{array}$


Echantilion 2

\begin{tabular}{|c|c|c|c|c|c|}
\hline & .6 & .8 & 1.0 & 1.2 & 1.4 \\
\hline Si & -33.40 & -20.84 & -8.29 & 4.26 & 16.82 \\
\hline$A 7$ & -6.48 & -3.62 & -0.77 & 2.08 & 4.93 \\
\hline $\mathrm{Fe}$ & 3.36 & 5.04 & 6.73 & 8.42 & 10.11 \\
\hline $\mathrm{Mg}$ & 0.23 & 0.44 & 0.65 & 0.86 & 1.07 \\
\hline $\mathrm{Ca}$ & 1.22 & 1.85 & 2.49 & 3.12 & 3.76 \\
\hline $\mathrm{Na}$ & 0.85 & 2.39 & 3.93 & 5.47 & 7.02 \\
\hline$k$ & -4.24 & -3.32 & -2.40 & -1.49 & -0.57 \\
\hline $\mathrm{Ti}$ & 0.02 & 0.10 & 0.19 & 0.28 & 0.37 \\
\hline P & 0.21 & 0.30 & 0.38 & 0.46 & 0.55 \\
\hline Mn & -.06 & 0.09 & 0.12 & 0.76 & 0.19 \\
\hline
\end{tabular}

Tableau 1. Valeur de Xn pourF $v=0.6,0.8,1.0,1.2$ et 1.4, échantillon 2 par rapport à l'échantilion 14 . 


\begin{tabular}{|c|c|c|c|c|c|c|c|c|c|c|c|c|c|c|}
\hline & 1 & 2 & 3 & 4 & 5 & 6 & 7 & 8 & 9 & 10 & 11 & 12 & 13 & 14 \\
\hline & 96734 & 57821 & 57877 & 57879 & 56071 & 56070 & 8 & 96733 & 56174 & 57471 & 57479 & 57506 & 56175 & 57515 \\
\hline $\mathrm{SiO}_{2}$ & 55.8 & 55.8 & 57.2 & 50.4 & 54.0 & 52.5 & 53.34 & 49.2 & 54.38 & 51.18 & 54.98 & 49.03 & 55.22 & 56.51 \\
\hline $\mathrm{Al}_{2} \mathrm{O}_{3}$ & 20.5 & 22.0 & 27.6 & 24.3 & 22.50 & 20.30 & 20.10 & 22.2 & 25.86 & 22.61 & 24.93 & 20.18 & 23.23 & 23.05 \\
\hline $\mathrm{Fe}_{2} \mathrm{O}_{3}$ & 2.15 & 1.08 & 0.71 & 0.42 & 0.50 & 0.59 & 3.58 & 0.58 & 0.51 & 0.05 & 0.12 & 0.64 & $\cdots$ & $\ldots$ \\
\hline $\mathrm{FeO}$ & 1.87 & 1.42 & 1.87 & 0.35 & 1.00 & 1.54 & 2.34 & $0 . \dot{84}$ & 0.66 & 1.67 & 1.46 & 2.46 & 1.20 & 1.29 \\
\hline $\mathrm{MgO}$ & 0.40 & 0.30 & 0.60 & 0.20 & 0.29 & 0.58 & 0.78 & 0.60 & 0.39 & 0.75 & 0.42 & 1.36 & 0.18 & 0.26 \\
\hline $\mathrm{CaO}$ & 1.12 & 1.02 & 1.90 & 3.60 & 2.45 & 4.84 & 2.40 & 6.50 & 0.51 & 3.96 & 0.53 & 6.25 & 0.63 & 1.03 \\
\hline $\mathrm{Na}_{2} \mathrm{O}$ & 7.7 & 9.2 & 7.3 & $\therefore \quad 11.4$ & 11.25 & 10.25 & 8.46 & 8.50 & $11.42^{\circ}$ & 9.35 & 10.49 & 8.21 & 12.22 & 11.35 \\
\hline $\mathrm{K}_{2} \mathrm{O}$ & 6.95 & 5.75 & 4.65 & 4.60 & 4.00 & 4.00 & 5.77 & 3.40 & 4.79 & 4.53 & 4.60 & 4.72 & 3.64 & 3.50 \\
\hline $\mathrm{TiO}_{2}$ & 0.68 & 0.37 & 0.40 & 0.09 & 0.25 & 0.29 & 6.83 & 0.30 & 0.16 & 0.11 & 0.11 & 0.55 & 0.14 & 0.11 \\
\hline $\mathrm{P}_{2} \mathrm{O}_{5}$ & 0.10 & 0.05 & 0.22 & 1.23 & 0.36 & 0.36 & 0.25 & 0.22 & 0.09 & 0.43 & 0.09 & 0.78 & 0.06 & 0.14 \\
\hline Mno & 0.03 & 0.04 & 0.04 & 0.03 & 0.03 & 0.06 & 0.23 & 0.07 & $-\cdots$ & --- & --- & --- & --- & ---- \\
\hline $\mathrm{CO}_{2}$ & 1.25 & 0.92 & 0.92 & 1.66 & 1.20 & 3.30 & $\cdots$ & 4.40 & $(p . a . f)$. & $(p . a . f)$ & $(p \cdot a . f)$ & $(p . a . f)$ & $(p . a . f)$ & $(p . a . f)$ \\
\hline $\mathrm{H}_{2} \mathrm{O}_{\mathrm{T}}$ & 1.11 & 0.90 & 0.90 & 1.06 & 1.90 & 1.65 & 1.22 & 1.92 & 0.97 & 4.76 & 1.54 & 5.19 & 1.14 & 1.30 \\
\hline TOTAL: & 99.66 & 98.85 & 98.89 & 99.34 & 99.73 & 100.26 & 99.30 & 98.73 & 99.76 & 99.46 & 98.30 & 99.43 & 97.74 & 98.82 \\
\hline $\mathrm{Rb}$ & 74 & 130 & 140 & 80 & $\cdots-$ & $---\cdots$ & $-\cdots$ & 62 & $\cdots$ & $\ldots$ & $\ldots-$ & $\cdots+$ & $\cdots-$ & $\cdots$ \\
\hline Sn & 880 & 860 & 1300 & 1100 & $\cdots-$ & $-\cdots-\cdots$ & $\ldots$ & 2300 & $-\cdots$ & $\ldots-$ & $\cdots$ & $-\cdots-$ & $-\cdots-$ & ---- \\
\hline $\mathrm{Nb}$ & 168 & 86 & 83 & 8 & $-\cdots$ & $\cdots-\cdots$ & $\cdots--$ & 7600 & $\ldots$ & $\ldots-$ & $\cdots+$ & $\ldots$ & $\cdots$ & $\cdots-$ \\
\hline $\mathrm{Ba}$ & 1240 & 670 & 2200 & 580 & - - - & $\ldots-\cdots$ & $\cdots$ & 405 & $-\cdots-$ & $\cdots-$ & $\ldots-$ & $\cdots$ & $\ldots$ & $\cdots$ \\
\hline U & 2 & 6 & 4 & 2 & $-\cdots-$ & $---\cdots$ & $\cdots$ & 2 & $\cdots-$ & $\cdots--$ & --- & $-\cdots$ & --- & --- \\
\hline Th & 5 & 5 & 5 & 5 & $-\cdots-$ & $-\cdots$ & ---- & 35 & $\cdots$ & $\cdots-$ & --- & $-\ldots-$ & ---- & $-\cdots-\infty$ \\
\hline Ta & 20 & 20 & 20 & 20 & $-\cdots$ & $(--\infty$ & $\cdots$ & 350 & … & $\cdots$ & $-\cdots$ & $\ldots$ & $-\cdots$ & --- \\
\hline
\end{tabular}




\begin{tabular}{|c|c|c|c|c|c|c|c|c|c|c|c|c|c|c|}
\hline & 15 & 16 & 17 & 18 & 19 & 20 & 21 & 22 & 23 & 24 & 25 & 26 & 27 & 28 \\
\hline & $57857^{\circ}$ & , 57858 & 56006 & 56072 & . 56073 & . 56074 & 96729 & 96731 & . 96732 & . 96730 & 96727 & , 96728 & .56007 & . 9672 \\
\hline $\mathrm{SiO}_{2}$ & 32.8 & 56.4 & 50.0 & 43.4 & 49.5 & 50.1 & 55.0 & 53.4 & 57.6 & 56.8 & 35.8 & 39.8 & 35.7 & 1.3 \\
\hline $\mathrm{Al}_{2} \mathrm{O}_{3}$ & 21.0 & 22.7 & 19.0 & 16.60 & 16.40 & 15.65 & 15.80 & 22.8 & 21.2 & 22.4 & 11.1 & 11.6 & 11.30 & 0.2 \\
\hline $\mathrm{Fe}_{2} \mathrm{O}_{3}$ & 1.53 & 0.74 & 0.80 & 1.40 & 1.64 & 0.91 & 0.59 & 1.28 & 0.86 & 0.68 & 1.79 & 2.76 & 2.10 & 0.5 \\
\hline $\mathrm{Fe} 0$ & 1.42 & 1.09 & 2.30 & 3.47 & 3.47 & 2.95 & 1.16 & 2.38 & 2.64 & 1.67 & 6.37 & 10.16 & 9.30 & 0.9 \\
\hline MgO & 0.40 & 0.90 & 0.75 & 0.97 & 1.06 & 0.75 & 0.40 & 0.60 & 0.20 & 0.30 & 4.5 & 6.8 & 3.18 & 1.4 \\
\hline $\mathrm{CaO}$ & 4.80 & 2.60 & 5.70 & 12.70 & 9.75 & 10.90 & 8.20 & 1.28 & 0.74 & 1.38 & 16.4 & 8.50 & 13.0 & 52.9 \\
\hline $\mathrm{Na}_{2} \mathrm{O}$ & 8.90 & 6.70 & 7.00 & 6.80 & 6.55 & 6.10 & 6.20 & 10.30 & 8.8 & 8.7 & 4.2 & 3.50 & 3.50 & 1.0 \\
\hline $\mathrm{K}_{2} \mathrm{O}$ & 4.40 & 4.40 & 6.80 & 4.40 & 4.55 & 4.95 & 5.15 & 5.25 & 5.20 & 4.05 & 3.55 & 5.35 & 4.85 & 0.1 \\
\hline $\mathrm{TiO}_{2}$ & 0.34 & 0.26 & 0.52 & 0.85 & 1.19 & 0.56 & 0.26 & 0.56 & 0.49 & 0.30 & 1.75 & 3.30 & 4.80 & 0.0 \\
\hline$P_{2} O_{5}$ & 1.30 & 0.18 & 0.08 & 0.86 & 0.54 & 0.31 & 0.70 & 0.22 & 0.12 & 0.22 & 2.98 & 1.30 & 0.39 & 0.0 \\
\hline MnO & 0.08 & 0.06 & 0.07 & 0.13 & 0.12 & 0.11 & 0.09 & 0.05 & 0.09 & 0.05 & 0.16 & 0.17 & 0.18 & 0.3 \\
\hline $\mathrm{CO}_{2}$ & 2.75 & 0.88 & 4.60 & 6.70 & 4.70 & 6.10 & 5.74 & 0.88 & 0.34 & 0.47 & 10.31 & 5.53 & 7.65 & 39.0 \\
\hline $\mathrm{H}_{2} \mathrm{O}_{\mathrm{T}}$ & 1.25 & 2.76 & 0.86 & 1.45 & 1.00 & 0.96 & 0.59 & 1.20 & 0.41 & 1.39 & 1.28 & 1.35 & 1.64 & 0.3 \\
\hline TOTAL: & 100.97 & 99.67 & 98.48 & 99.73 & 100.47 & 100.35 & 99.88 & 100.2 & 98.69 & 98.41 & 100.19 & 100.12 & 97.49 & 98.2 \\
\hline$R b$ & 110 & 100 & 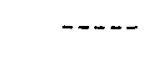 & ---- & $-\cdots$ & 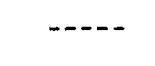 & 84 & 140 & 170 & 120 & 130 & 110 & $-\cdots$ & 11 \\
\hline Sn & 1300 & 1700 & $\cdots$ & $-\cdots$ & $-\cdots$ & $n^{-\cdots-}$ & 1800 & 1260 & 1200 & 1400 & 1800 & 1550 & $\ldots$ & 9600 \\
\hline $\mathrm{Nb}$ & 60 & 37 & $\cdots$ & $\cdots--$ & ---- & $\ldots-$ & 31 & 94 & 200 & 170 & 260 & 210 & $\ldots$ & 4 \\
\hline $\mathrm{Ba}$ & 1240 & 1120 & $\ldots-$ & $-\cdots$ & $\cdots-$ & $\ldots$ & 1060 & 1450 & 1780 & 890 & 415 & 540 & $\ldots$ & 125 \\
\hline U & 2 & 2 & $\cdots$ & $\ldots$ & $\ldots$ & $\cdots$ & 2 & 2 & 6 & 4 & 2 & 2 & $\cdots$ & 2 \\
\hline Th & 5 & 6 & $\cdots--$ & $\cdots-$ & $\cdots-$ & $\cdots-$ & 5 & 5 & 9 & 10 & 9 & 6 & $-\cdots$ & 9 \\
\hline $\mathrm{Ta}$ & 20 & 20 & $\cdots-$ & $\cdots--$ & $\cdots-$ & $\cdots \cdots$ & 20 & 20 & 20 & 20 & 20 & 20 & $\cdots$ & 20 \\
\hline
\end{tabular}


CALCUL DE LA NORME DES ROCHES DU COMPLEXE

\begin{tabular}{|c|c|c|c|c|c|c|c|c|c|c|c|c|c|c|}
\hline & 1 & 2 & 3 & 4 & 5 & 6 & 7 & 8 & 9 & 10 & $\pi$ & 12 & $\sqrt{3}$ & 74 \\
\hline & SN & $\overline{S N}$ & SN & $\overline{S N}$ & SN & SN & $\mathrm{SN}$ & SN & $\mathrm{SN}$ & SN & $\overline{S N}$ & $S N$ & SN & SN \\
\hline & I & II & I & I & II & I & II & II & II & II & II & II & I I & II \\
\hline$\overline{\mathrm{QZ}}$ & $\cdots$ & $\ldots$ & $\ldots$ & $\ldots$ & $\ldots$ & $\ldots$ & $\ldots$ & -... & $\ldots$ & $\ldots$ & $\cdots$ & $\cdots$ & $\ldots$ & $\cdots$ \\
\hline $\mathrm{OR}$ & 33.98 & 27.18 & 41.07 & 20.09 & 23.64 & 23.64 & 27.48 & 28.31 & 26.77 & 27.18 & 27.89 & 21.51 & 20.68 & 26.00 \\
\hline $\mathrm{PL}$ & 31.74 & 18.67 & 27.40 & 32.39 & 29.00 & 22.95 & 54.58 & 29.56 & 24.61 & 27.21 & 15.40 & 36.24 & 42.82 & 33.68 \\
\hline AN & 1.75 & 1.55 & 0.85 & .12 .38 & --- & $-\cdots$ & 7.99 & 1.94 & 6.34 & 2.04 & 4.27 & -.-- & 1.61 & 4.36 \\
\hline$A B$ & 22.99 & 17.12 & 26.55 & 20.01 & 29.00 & 22.95 & 46.60 & 27.62 & 18.26 & 25.17 & 11.13 & 36.24 & 41.21 & 29.32 \\
\hline NE & 25.93 & 42.98 & 20.91 & 28.13 & 34.92 & 32.07 & 8.22 & 37.39 & 32.97 & 34.45 & 31.60 & 34.12 & 29.70 & 24.91 \\
\hline LE & $\ldots$ & $\ldots-$ & $-\cdots$ & $-\cdots$ & $\cdots$ & $\ldots$ & --- & $-\cdots$ & $-\cdots$ & $-\cdots$ & $\ldots$ & --- & --- & $\ldots$ \\
\hline$A C$ & $\cdots$ & $\cdots-$ & $\ldots$ & $\cdots$ & 1.45 & 1.71 & -..... & ---- & -...- & -... & $-\cdots$ & $-\cdots$ & ----- & $\cdots$ \\
\hline NS & $\ldots$ & $\cdots$ & $\cdots$ & $\cdots$ & 0.02 & 0.62 & -..... & ----- & ----- & -...- & $\cdots$ & 0.97 & --- & $\cdots$ \\
\hline wo & $\cdots$ & 2.70 & 0.02 & 5.35 & 1.95 & 5.21 & $-\cdots$ & $-\cdots$ & $-\cdots$ & $\cdots$ & 2.40 & $-\cdots$ & $-\cdots$ & 2.61 \\
\hline DISS & 2.51 & 1.46 & 3.26 & 4.54 & 4.34 & 7.74 & $\ldots$ & $\cdots$ & 8.90 & $\cdots$ & 13.10 & 2.37 & 2.22 & 3.90 \\
\hline DI & 1.06 & 1.07 & 2.15 & 3.22 & 1.56 & 3.12 & ---- & $\ldots$ & 3.78 & ---- & 7.31 & 0.49 & 0.56 & 2.15 \\
\hline HE & 1.45 & 0.38 & 1.11 & 1.31 & 2.78 & 4.63 & -..-- & $\ldots$ & 5.02 & ---.- & 5.79 & 1.88 & 1.66 & 1.75 \\
\hline $\mathrm{OL}$ & 0.50 & $-\cdots$ & $\cdots$ & $\cdots$ & --.- & ---- & 1.05 & 1.09 & 0.21 & 35.35 & ----- & 0.91 & 1.28 & $\cdots$ \\
\hline F0 & 0.18 & ---- & -..-- & $\ldots$ &.-- & $\ldots . .$. & 1.05 & 0.68 & 0.08. & 0.73 & $\because \quad \cdots . .$. & 0.16 & 0.27 & $\ldots$ \\
\hline FA & 0.31 & --- & $\cdots-$ & 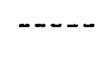 & $\cdots$ & $\cdots--$ & $\cdots$ & 0.41 & 0.13 & 4.62 & $-\cdots-$ & 0.75 & 1.01 & --- \\
\hline MT & 1.57 & 0.61 & 3.12 & 0.84 & ---- & $\cdots$ & 0.91 & 0.74 & 0.07 & 0.17 & 0.93 & $\cdots--$ & --.-- & 2.22 \\
\hline AU & $\ldots$ & $\cdots+$ & $-\cdots-$ & -..-- & ----- & $-\cdots-$ & .0 .09 & $\cdots$ & $\cdots$ & -..- & $\cdots$ & $\cdots$ & ---- & $\cdots$ \\
\hline IL & 0.70 & 0.17 & 1.29 & 0.57 & 0.47 & 0.55 & 0.86 & 0.30 & 0.21 & 0.21 & 1.04 & 0.27 & 0.21 & 0.65 \\
\hline AD & 0.12 & 2.90 & 0.24 & 0.52 & 0.85 & 0.85 & 0.52 & 0.21 & 1.01 & 1.01 & 1.84 & 0.14 & 0.33 & 3.07 \\
\hline${ }^{\mathrm{H}_{2}} \mathrm{O}_{\mathrm{T}}$ & 0.90 & 1.06 & 1.11 & 1.92 & 1.90 & 1.65 & 1.06 & 1.66 & 6.12 & 6.12 & 6.39 & 1.14 & --- & 0.0 \\
\hline TOTAL & 97.93 & 97.73 & 98.41 & 94.34 & 98.55 & 96.98 & 97.40 & 100.43 & 100.78 & 99.83 & 100.60 & 97.66 & 97.75 & 97. \\
\hline
\end{tabular}


CALCUL DE LA NORME DES ROCHES DU COMPLEXE

\begin{tabular}{|c|c|c|c|c|c|c|c|c|c|c|c|c|}
\hline 15 & 16 & 17 & 78 & 19 & 20 & 21 & 22 & 23 & 24 & 26 & 27 & 28 \\
\hline $\mathrm{SN}$ & SN & SN & SN & $S$ & $S$ & SN & SN & SN & SB & SB & SB & C \\
\hline I I I & III & II I & II I & & & IV & IV & IV & & & & \\
\hline---- & $\cdot-\cdot--$ & $\cdots-$ & $-\cdots$ & -...- & 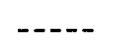 & ..... & $\ldots$ & $-\ldots$ & $\ldots-$ & $-\ldots$ & --- & $-\cdots$ \\
\hline 26.00 & 40.18 & 13.53 & 26.89 & 30.43 & 29.25 & 23.93 & 31.02 & 31.73 & $\ldots$. & 3.76 & $\ldots$. & $\ldots$ \\
\hline 56.66 & 4.55 & 1.78 & 15.48 & 31.13 & 11.63 & 52.09 & 23.79 & 43.14 & 0.95 & 0.14 & 0.80 & $\ldots-$. \\
\hline 11.72 & 0.34 & 1.78 & 1.91 & 0.07 & 0.65 & 5.41 & 0.47 & 2.89 & 0.95 & 0.14 & 0.80 & $-\cdots$ \\
\hline 44.93 & 4.21 & $\ldots$ & 13.57 & 31.06 & 10.98 & 46.68 & 23.31 & 40.25 & $-\cdots-$ & $\cdots-$ & ---- & $\cdots$ \\
\hline 6.37 & 29.81 & 31.17 & 22.67 & 11.60 & 22.01 & 14.59 & 34.59 & 18.53 & 19.25 & 16.04 & 16.04 & 0.10 \\
\hline$\cdots$ & $-\ldots$ & 9.78 & -...- & -... & $\ldots-$ & .... & $-\ldots$ & -... & 16.45 & 21.84 & 22.47 & 80.58 \\
\hline$\cdots$ & $-\cdots$ & $\cdots-$ & $\ldots$ & $\ldots-$ & $-\cdots$ & $\cdots-$ & $-\cdots$ & $-\cdots-$ & ---- & $\ldots-$ & $-\cdots$ & 1.71 \\
\hline$-\cdots$ & ---- & $\cdots-$ & $\cdots$ & $-\ldots$ & 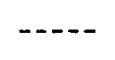 & $\ldots$ & $\cdots$ & $\ldots-$ & -...- & $\ldots$ & --- & 1.47 \\
\hline$-\cdots$ & 6.79 & 16.85 & 11.99 & 12.68 & 15.83 & -..- & $\ldots$ & $\ldots-$ & --- & $-\cdots$ & --- & --- \\
\hline$-\cdots$ & 9.36 & 12.83 & 11.85 & 4.75 & 11.45 & 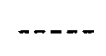 & 3.74 & $\cdots$ & 24.66 & 27.38 & 21.04 & $-\ldots$ \\
\hline---- & 4.03 & 5.21 & 5.69 & 2.15 & 4.03 & .... & 1.53 & $\ldots$ & 15.48 & 17.34 & 11.48 & $\ldots$ \\
\hline$\cdots-$ & 5.33 & 7.62 & 6.16 & 2.60 & 7.42 & $\cdots$ & 2.21 & $\cdots$ & 9.18 & 10.04 & 8.56 & $\cdots-$ \\
\hline 2.40 & $\ldots-$ & $\cdots-$ & $\cdots$ & $\cdots$ & ---- & 2.15 & 1.56 & 3.05 & 4.94 & 10.80 & 3.74 & 4.21 \\
\hline 1.57 & $\ldots-$ & ....- & $-\cdots$ & $-\cdots-$ & $-\ldots-$ & 0.52 & 0.55 & 0.35 & 2.83 & 6.23 & 4.99 & 2.44 \\
\hline 0.83 & $\cdots-$ & $\ldots$ & $\ldots$ & ---- & $-\cdots$ & 1.62 & 1.01 & 2.70 & 2.12 & 4.56 & 1.92 & 1.77 \\
\hline 1.07 & 1.16 & 2.03 & 2.38 & 0.86 & 1.32 & 0.99 & 7.86 & 1.25 & 2.60 & 4.00 & 3.04 & -..- \\
\hline$\ldots-\cdots$ & $\cdots$ & ---- & -...- & 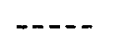 & $--\ldots$ &.--- & $-\ldots$ &.--- & $\mathrm{CS}$ & $\mathrm{CS}$ & 11.05 & ..... \\
\hline 0.49 & 0.99 & 1.61 & 2.26 & 0.49 & 1.06 & 0.57 & 1.06 & 0.93 & 3.32 & 6.27 & 9.12 & 0.04 \\
\hline 0.42 & 0.19 & 2.03 & 1.27 & 1.65 & 0.73 & 0.52 & 0.52 & 0.28 & 7.03 & 3.07 & 0.92 & 0.05 \\
\hline 0.03 & 0.01 & 0.01 & 0.01 & 0.59 & 0.96 & 1.39 & 1.20 & 0.41 & 1.20 & 1.35 & 1.64 & 0.37 \\
\hline 96.94 & 97.63 & 98.29 & 99.48 & 94.17 & 94.24 & 97.25 & 99.36 & 93.36 & 90.01 & 94.65 & 89.86 & 59.23 \\
\hline
\end{tabular}




\section{SOURCE DES ECHANTILLONS}

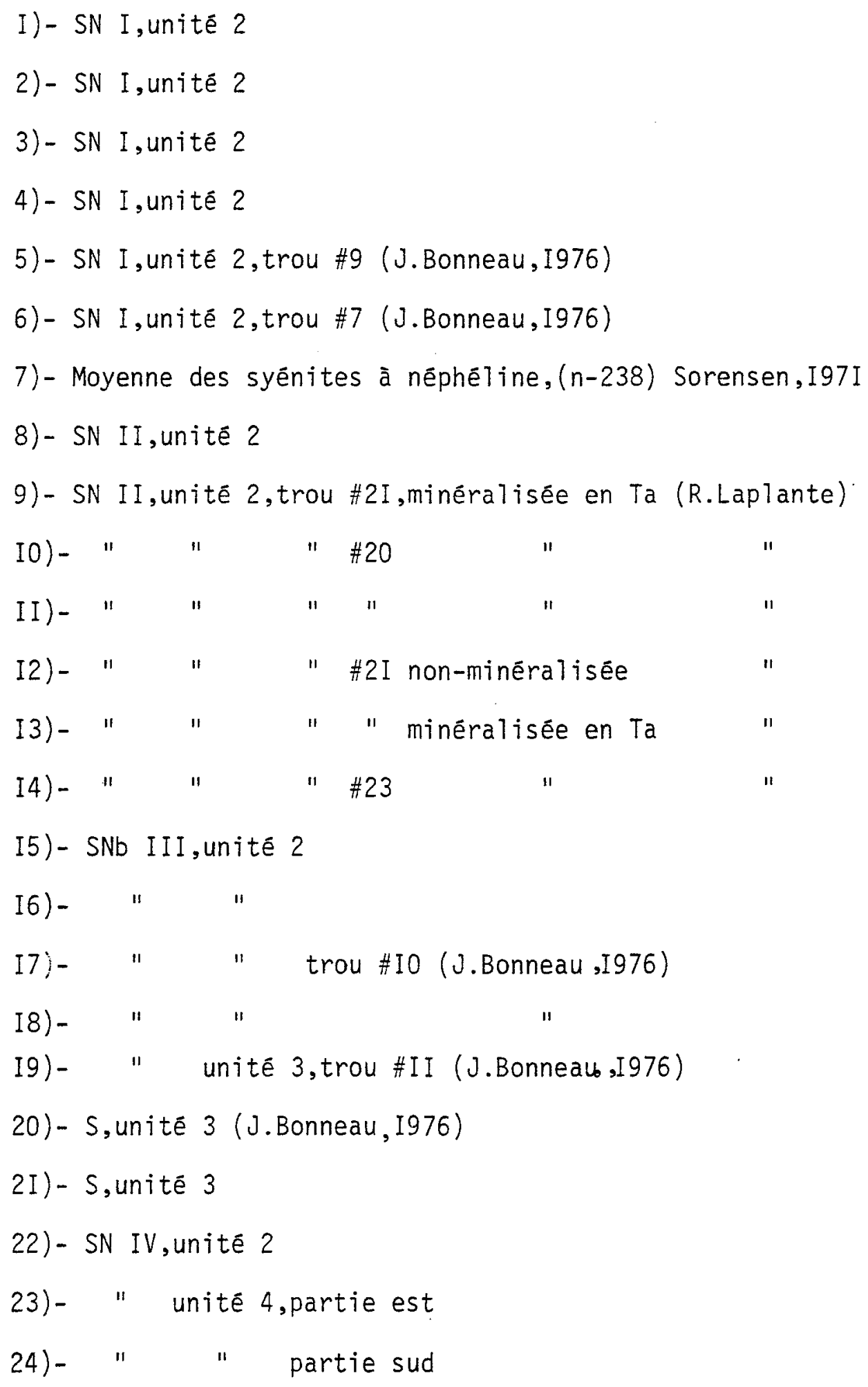


25)- SNb III, unité I

$\begin{array}{lll}26)- & " 1 & \\ 27)- & " & \text { (J.Bonneau, 1976) } \\ 28)- & " 1 \\ \text { 29)- C, unite I }\end{array}$




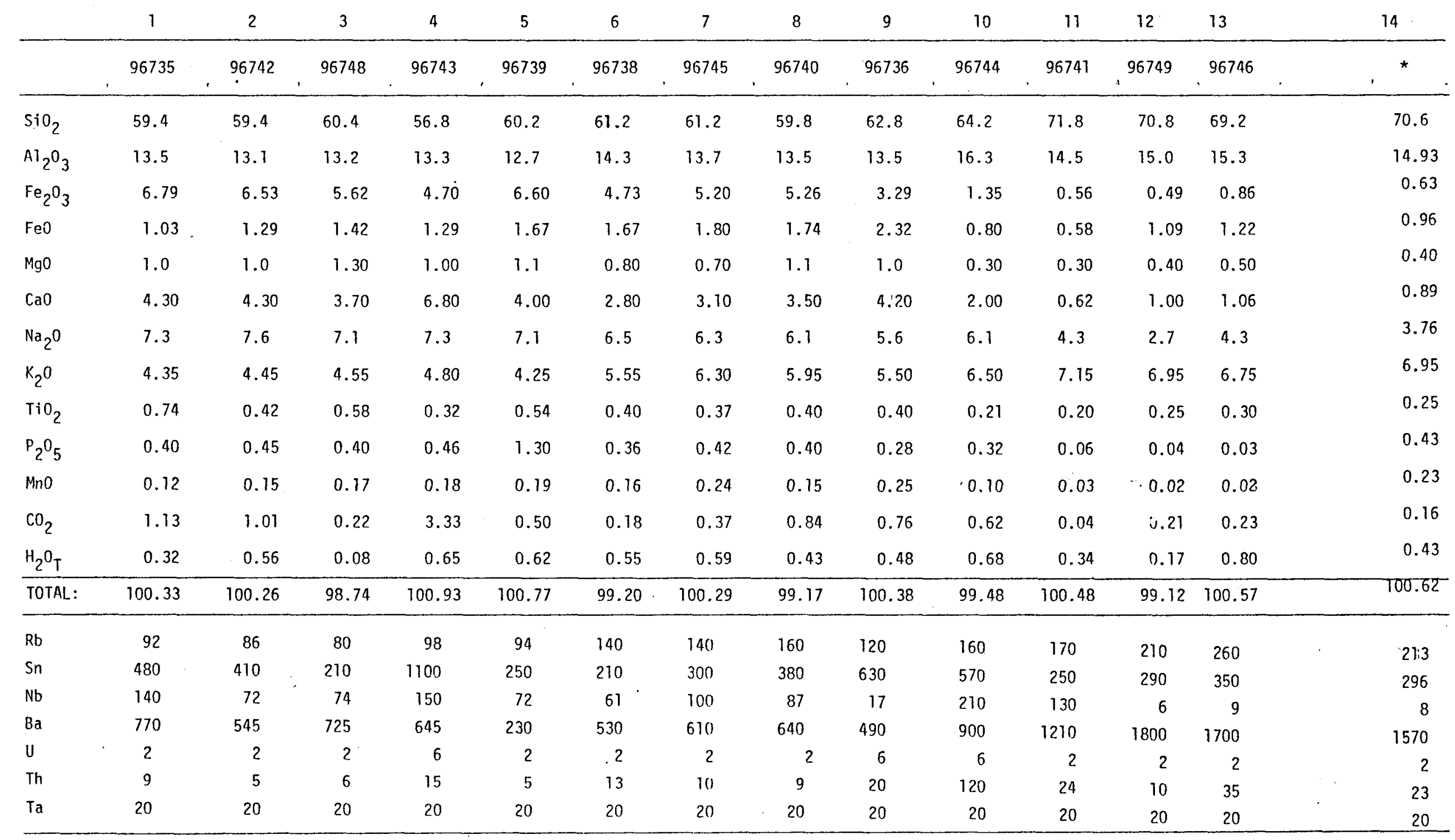




\begin{tabular}{|c|c|c|c|c|c|c|c|c|c|c|c|c|c|}
\hline & 7 & 2 & 3 & 4 & 5 & $\overline{6}$ & 7 & $\overline{8}$ & 9 & $1 \overline{10}$ & 11 & 12 & 13 \\
\hline & 96735 & 96742 & 96748 & 96743 & 96739 & 96738 & III 96745 & 96740 & 96744 & 96736 & 96741 & 96749 & 96746 \\
\hline$Q Z$ & ---- & $\cdots$ & $\ldots$ & $\cdots$ & 1.99 & 0.43 & $\ldots-$ & $\ldots-$ & 2.07 & 3.95 & 18.43 & 25.32 & 15.49 \\
\hline$O R$ & 25.71 & 26.30 & 26.30 & 28.36 & 25.11 & 32.80 & 37.23 & 35.16 & 38.41 & 32.50 & 42.25 & 41.07 & 39.89 \\
\hline$P L$ & 42.06 & 35.02 & 42.20 & 25.95 & 41.66 & 42.66 & 34.60 & 34.51 & 47.65 & 38.82 & 34.78 & 27.55 & 38.90 \\
\hline AN & $-\cdots$ & $\ldots$ & $\cdots$ & $-\therefore-$ & $\cdots$ & -..- & $\cdots$ & $\cdots-$ & ---- & $\cdots--$ & $\cdots$ & 4.70 & 2.51 \\
\hline$A B$ & 42.06 & 35.02 & 42.20 & 25.95 & 41.66 & 42.66 & 34.60 & 35.51 & 47.65 & 38.82 & 34.78 & 22.85 & 36.39 \\
\hline$N E$ & 1.71 & 3.86 & $\cdots$ & 9.07 & $\cdots$ & $-\cdots$ & 0.43 & 0.98 & $\cdots--$ & $\cdots--$ & $-\cdots$ & ---- & $-\cdots$ \\
\hline LE & 14.58 & 18.89 & 15.75 & 13.60 & 16.22 & 10.88 & 15.04 & 13.48 & 3.49 & 7.55 & 1.42 & $--\cdots$ & ---- \\
\hline$A C$ & --- & 0.17 & $\cdots-$ & 1.08 & $\cdots-$ & - - & 0.19 & $\cdots$ & $\cdots$ & $\cdots--$ & -..- & $\cdots$ & $-\cdots$ \\
\hline NS & $-\cdots$ & $\ldots-$ & $\cdots$ & $\cdots$ & $\cdots$ & ---- & $-\cdots$ & $\cdots-$ & $\ldots$ & $\cdots-$ & $-\cdots$ & 1.31 & $-\cdots$ \\
\hline wo & 4.93 & 3.6 & 1.22 & 8.03 & 0.07 & 0.84 & 0.49 & 0.95 & 1.36 & 1.97 & ---- & ---- & ---- \\
\hline DISS & 5.37 & 9.05 & 10.41 & 9.46 & 9.12 & 7.87 & 9.67 & 10.27 & 3.85 & 11.96 & 2.22 & ---- & 2.10 \\
\hline DI & 5.37 & 5.37 & 6.98 & 5.37 & 5.91 & 4.30 & 3.76 & 5.91 & 1.61 & 5.37 & 1.20 & ---- & 1.20 \\
\hline $\mathrm{OL}$ & $-\cdots$ & $\ldots-$ & $\cdots$ & ---- & $\ldots-$ & ---- & $-\cdots$ & $\cdots$ & $\cdots$ & $-\cdots$ & $\ldots 0.38$ & 2.22 & 1.28 \\
\hline Fo & $\cdots$ & $-\cdots$ & $\cdots-$ & $\cdots-$ & $-\therefore-$ & ---- & $--\therefore$ & $\cdots$ & $\cdots-$ & $\cdots---$ & 0.19 & 1.00 & 0.69 \\
\hline FA & $\cdots$ & $\cdots-$ & $\cdots-$ & $\cdots--$ & $-\cdots$ & $\ldots$ & ---- & $-\cdots-$ & $-\ldots$ & ---- & 0.19 & 1.22 & 0.59 \\
\hline MT & 1.57 & ---- & 0.25 & ---- & 1.44 & 1.41 & $-\cdots$ & 0.87 & 0.21 & 0.99 & 0.10 & 0.71 & 1.25 \\
\hline AU & 0.62 & ---- & $\cdots$ & $\cdots$ & $\cdots-$ & $\ldots-$ & $\therefore-$ & $-\cdots$ & $-\cdots$ & $\cdots$ & $-\cdots$ & $-\cdots$ & ---- \\
\hline IL & 1.41 & 0.81 & 1.10 & 0.61 & 1.03 & 0.76 & 0.70 & 0.76 & 0.40 & 0.76 & 0.38 & 0.47 & 0.57 \\
\hline AP & 0.93 & 0.99 & 0.94 & 1.09 & 3.07 & 0.85 & 0.99 & 0.94 & 0.75 & 0.66 & 0.14 & 0.09 & 0.07 \\
\hline $\mathrm{H}_{2} \mathrm{O}_{\mathrm{T}}$ & 0.32 & 0.56 & 0.08 & 0.65 & 0.62 & 0.55 & 0.59 & 0.43 & 0.68 & 0.43 & 0.34 & 0.17 & 0.80 \\
\hline TOTAL: & 99.22 & 98.79 & 98.26 & 96.90 & 100.33 & 99.04 & 99.94 & 98.35 & 98.87 & 99.58 & 100.44 & 98.91 & 100.34 \\
\hline
\end{tabular}


SOURCE DES ECHANTILLONS

I)- Pegmatite métasomatisée, unité 4 ,zone de contact soit à $x=0$

2)-

3)-

4)-

5)-

6)-

7)-

8)-

9)-

I0)-
11

11

11

II

11

II

11

II

II $x=1000 \mathrm{pi}$.

$x=2000$ pi.

$x=3500 \mathrm{pi}$.

$x=5500$ pi.

$x=7000$ pi.

$x=7900 \mathrm{pi}$.

$x=8900 \mathrm{pi}$.

$x=9400$ pi.

$x=9800$ pi.

II)- Pegmatite faiblement métasomatisēe, unité 4 , encaissant $x=10400 \mathrm{pi}$.

I2)- Pegmatite non métasomatisée, unitë 4 ,encaissant

I3)-

"

I4)- Moyenne des analyses \#II,I2 et I3 


\section{REFERENCES}

Appleyard, E.C., et Wooley, A.R., 1979. Fenetization; an example of the problem of characterising mass transfer and volume change. Chem. Geol., 26: $1-15$

Aubertin, M., 1976. Rapport interne de la Soquem, projet Crevier.

Bailey, D.K., 1971. Continental rifting and alkaline magmatism. Dans: AlkaTine Rocks, Sorensen. John Wiley and Son, N.Y.

Barth, T.F.W., 1948. 0xygen in rocks, a basis for petrographic calculation. J. Geol., 56: 50-60

Bergeron, A., 1979. Rapport interne de la Soquem, projet Crevier. Bergeron, A. et Laplante, R., 1978. Rapport interne de la Soquem, projet Crevier.

Bogolepov, V.G., 1962. The recomputation of the chemical analyses of rock in studying metasomatic process. Int. Geol. Rev., Vol. 5, \#12, 1585-1592

Bonneau, J., 1977. Rapport interne de la Soquem, projet Crevier.

Borodin, L.S. et Pavlenko, A.S., 1971. The role of metasomatic process in the formation of alkaline rocks. Dans: Alkaline Rocks, Sorensen. John Wiley and Son, N.Y.

Bowen, N.L., 1928. The Evolution of Igneous Rocks. Dover Publication, N.Y., réedition $1956,332 \mathrm{p}$.

Currie, K.L., 1976. The Alkaline Rocks of Canada. GSC Bulletin 238.

Daly, R.A., 1933. Igneous rocks and the depth of the earth. Mc Graw-Hill, N.Y., 568 p. 
Douglas, R.J.W., 1968. Géologie et ressources minêrales du Canada, partie A, GSC, Séries de la géologie du Canada, \#1, 408 p.

Frith, R.A., 1971. Rb-Sr isotopic studies across the Grenville province, Québec. PhD Thesis, McGi11 University, $157 \mathrm{p}$.

Girault, J., 1966. Genèse et géochimie de l'apatite et de la calcite dans lesroches liées au complexe carbonatique et hyperalcalin d'Oka (Canada). Soc. Franc. Min. Bu11., Vo1. 84: 496-513

Gittins, J., 1973. The signifience of some porphyritic textures in carbonatite. Can. Min., Vol. 12: 226-228.

Gresens, R.L., 1967. Composition-Volume reliationships of metasomatism. Chem. Geo1., 2: 47-65.

Hamilton, D.L. et Mackenzie, W.S., 1965. Phase equilibrium studies in the system $\mathrm{NaAlSiO}_{4}$ (Nepheline)-KAlSiO 4 (Kalsilite)-SiO $-\mathrm{H}_{2} \mathrm{O}$. Min. Mag., Vol. 34: 214-231.

Heinrich, E.W., 1966. The geology of carbonatites. Rand and McNa17y, Chicago, $555 \mathrm{p}$.

Johannsen, A., 1938. A descriptive petrography of igneous rocks. Vol. IV, The Univ. of Chicago Press, $523 \mathrm{p}$.

Kerrich, R. et A7.,1977. Local Modification of Rock Chemestry by Deformation. Contrib. Mineral. Petrol., 65: 183-190.

Kogarko, L.N., 1971. Petrogenesis of Alkaline Rocks. Dans: Alkaline Rocks, Sorensen. John Wiley and Sons, N.Y. 
Kumarape11i, P.S. et Sall1, V.A., 1966. The St-Lawrence valley system: A North American equivalent of the East African rift valley system. C.J.E.S., Vol. 3: 639-656.

Luth, W.C., Jahns, R.H. et Tuttle, O.F., 1964. The granite system at pressures of 4 to 10 kilobars. Jour. Geoph. Res., Vol. 69, \#4: 759-773.

Mehnert, K.R., 1968. Migmatites and the origin of granitic rocks. Els. Publication Co., N.Y., 393 p.

Parsons, G.E., 1961. Niobium bearing complexes eat of Lake Superior. O.D.M. Geol. Report \#3: 23-32.

Philpotts, A.R., 1970. Mechanism of emplacement of the Monteregian intrusions. Can. Min., Vot. 10, \#3: 395-410.

Powe11, J.L. and Be11, K.,1971. Isotopic composition of strontium in alkalic rocks. Dans: Alkaline Rocks, Sorensen. John Wiley and Sons, N.Y.

Roubeault, M., 1963. Détermination des roches au microscope polarisant. Ed. Lamarre-Pincet, Paris, $363 \mathrm{p}$.

Roux, J. et Hamj]ton, D.L., 1976. Primary igneous analcite. An experimenta] study. Jour. of Geo1., Vo1. 17, \#2: 244-257.

Siemiatkowska, K.M. et Martin, R.F., 1975. Fenetization of Mississagi quartzite, Sudbury area, Ontario. Ge01. Soc. Am. Bu17., Vol. 86: 11091122.

Sharma, K.N.M. et Laurin, A.F., 1975. Rêgion des rivières Mistassini, Péribonca et Saguenay. Min. Rich. Nat., R.G. 161, 84 p. 
Smith, J.V., 1974. Feldspar mineral, Vol. 2, Chemical and mineral properties. Springer-Verlag, N.Y., 686 p.

Sorensen, H., 1971. The Alkaline Rocks. John Wiley and Sons, N.Y., 622 p.

Streckeisen, A., 1967. Classification and nomenclature of igneous roks. Neus Jah. fur Mineral., Abh. 107: \$44-240.

Tilley, C.F., 1967. Problems of alkaline rock genesis. Q. Jour. Soc. Lond., 323-360.

Wyllie. P.S. and Boettcher, A.L., 1969. Liquidus phase reliationship in the system $\mathrm{CaO}-\mathrm{CO}_{2}-\mathrm{H}_{2} \mathrm{O}$ to 4 kilobars pressure with petrological application. Am. Jour. of Science Schaerer, Vol. 267-A: 489-508.

Wyllie, P.S. and Tuttle, O.F., 1960. The system $\mathrm{CaO}-\mathrm{CO}_{2}-\mathrm{H}_{2} \mathrm{O}$ and the origin of carbonatites. Jour. of Petrol., Vol I: 1-46.

Wynne-Edwards, H.R., 1972. The Grenville province. Dans: Variation of Tectonic Styles in Canada. GAC Special Paper \# 11: 263-314. 